\title{
UNIVERSAL AND EXOTIC GENERALIZED FIXED-POINT ALGEBRAS FOR WEAKLY PROPER ACTIONS AND DUALITY
}

\author{
ALCIDES BUSS AND SIEGFRIED ECHTERHOFF
}

\begin{abstract}
Given a $C^{*}$-dynamical system $(A, G, \alpha)$, we say that $A$ is a weakly proper $X \rtimes G$-algebra if there exists a proper $G$-space $X$ together with a nondegenerate $G$-equivariant *-homomorphism $\phi: C_{0}(X) \rightarrow \mathcal{M}(A)$. Weakly proper $G$-algebras form a large subclass of the class of proper $G$-algebras in the sense of Rieffel. In this paper we show that weakly proper $X \rtimes G$-algebras allow the construction of full fixed-point algebras $A_{u}^{G, \alpha}$ corresponding to the full crossed product $A \rtimes_{\alpha} G$, thus solving, in this setting, a problem stated by Rieffel in his 1988's original article on proper actions. As an application we obtain a general Landstad duality result for arbitrary coactions together with a new and functorial construction of maximalizations of coactions.

The same methods also allow the construction of exotic generalized fixedpoint algebras associated to crossed-product norms lying between the reduced and universal ones. Using these, we give complete answers to some questions on duality theory for exotic crossed products recently raised by Kaliszewski, Landstad and Quigg.
\end{abstract}

\section{INTRODUCTION}

If a locally compact group $G$ acts properly on a locally compact space $X$, then we call a $C^{*}$-algebra $A$ a weakly proper $X \rtimes G$-algebra if there exists an action $\alpha: G \rightarrow \operatorname{Aut}(A)$ together with a $G$-equivariant nondegenerate *-homomorphism $\phi: C_{0}(X) \rightarrow \mathcal{M}(A)$. The notion of weakly proper $X \rtimes G$-algebras is much weaker than the notion of an action of the proper groupoid $X \rtimes G$, which requires that $\phi$ takes values in the center $\mathcal{Z} \mathcal{M}(A)$ of the multiplier algebra $\mathcal{M}(A)$. On the other hand, the notion of weakly proper $X \rtimes G$-algebras is stronger than Rieffel's notion of proper $G$-algebras (or proper actions on $C^{*}$-algebras) as introduced in [28. In fact, as shown by Rieffel in [29, Proposition 5.7], the dense subalgebra $A_{c}:=\phi\left(C_{c}(X)\right) A \phi\left(C_{c}(X)\right)$ carries a natural $A \rtimes_{\alpha, r} G$-valued inner product and the (reduced) generalized fixed-point algebra $A_{r}^{G, \alpha}$ is just the algebra of compact operators of the module $\mathcal{F}(A)_{r}$ which denotes the completion of $A_{c}$ as a Hilbert $A \rtimes_{\alpha, r} G$-module. Rieffel's generalized fixed-point algebra can be realized as the closure in $\mathcal{M}(A)$ of the fixed-point algebra with compact supports

$$
A_{c}^{G, \alpha}:=\phi\left(C_{c}(G \backslash X)\right) \widetilde{A}_{c}^{G, \alpha} \phi\left(C_{c}(G \backslash X)\right)
$$

with

$$
\widetilde{A}_{c}^{G, \alpha}=\left\{m \in \mathcal{M}(A)^{G, \alpha}: m \phi(f), \phi(f) m \in A_{c} \text { for all } f \in C_{c}(X)\right\}
$$

where $\mathcal{M}(A)^{G, \alpha}$ denotes the classical fixed-point algebra in $\mathcal{M}(A)$ under the extended action.

2010 Mathematics Subject Classification. 46L55, 22 D35.

Key words and phrases. weakly proper action, generalized fixed-point algebra, exotic crossed product, coactions, Landstad duality.

Supported by Deutsche Forschungsgemeinschaft (SFB 878, Groups, Geometry \& Actions), CNPq (Ciências sem Fronteira) and PVE/CAPES - Brazil.

We would like to thank the referee for the valuable suggestions which helped us to improve this paper. 
Looking at these results, it seems at first sight that the theory of generalized fixedpoint algebras in relation to the crossed product of $A$ with $G$ makes only sense for reduced crossed products. In fact, as observed by Rieffel in [28, pp. 145-146], it is not clear for general (Rieffel-) proper actions, whether the $L^{1}(G, A)$-valued inner product on $A_{c}$ satisfies the positivity condition for $C^{*}$-valued inner products when mapped into the full crossed product $A \rtimes_{\alpha} G$. On the other hand, in the recent paper [16], it is shown that for certain proper actions on $C^{*}$-algebras which can be realized as cross-sectional algebras of Fell bundles over certain groupoids, the inner product with values in the maximal crossed product does make sense, and it leads to a universal version of Rieffel's generalized fixed-point algebra in these cases. It lies in the nature of cross-sectional algebras of Fell bundles that the results in [16] are technically quite challenging. Also, one observes that all examples considered in [16] are weakly proper $X \rtimes G$-algebras in our sense for suitable $X$ (see Step 1 in the proof of [16, Proposition 3.3]).

In this paper we show that, indeed, for every weakly proper $X \rtimes G$-algebra $A$, the canonical $C_{c}(G, A)$-valued inner product on $A_{c}$ gives a well-defined inner product with values in $A \rtimes_{\alpha} G$ and hence $A_{c}$ completes to a Hilbert $A \rtimes_{\alpha} G$-module $\mathcal{F}_{u}(A)$. The $C^{*}$-algebra of compact operators on $\mathcal{F}_{u}(A)$ is realized as a completion $A_{u}^{G, \alpha}$ of the generalized fixed-point algebra with compact supports $A_{c}^{G, \alpha}$ of (1.1) and we call $A_{u}^{G, \alpha}$ the universal generalized fixed-point algebra of $A$. We say that $A$ is saturated, if the $A \rtimes_{\alpha} G$-valued inner product on $\mathcal{F}_{u}(A)$ is full, in which case $\mathcal{F}_{u}(A)$ becomes a $A_{u}^{G, \alpha}-A \rtimes_{\alpha} G$ equivalence bimodule. This is always the case if the action of $G$ on $X$ is free and proper, but this is not a necessary condition. In general $\mathcal{F}_{u}(A)$ will be a partial $A_{u}^{G, \alpha}-A \rtimes_{\alpha} G$ equivalence bimodule implementing a Morita equivalence between $A_{u}^{G, \alpha}$ and a suitable ideal in $A \rtimes_{\alpha} G$.

Hence the universal theory of generalized fixed-point algebras always works in the case of weakly proper actions. Although a lot of progress has been done recently in the setting of generalized fixed-point algebras for weakly proper actions (e.g. see [2, 3, 18]), it is quite surprising that this fact has not been noticed so far.

Our methods also allow the construction of generalized fixed-point algebras associated to exotic crossed products, meaning $C^{*}$-completions of $C_{c}(G, A)$ lying between $A \rtimes_{u} G$ and $A \rtimes_{r} G$, as discussed in a recent paper by Kaliszewski, Landstad and Quigg [15] and motivated by an earlier study of exotic group algebras by Brown and Guentner [6]. In the second part of this article we use this kind of generalized fixed-point algebra for weakly proper $G \rtimes G$-algebras (in which $G$ acts on itself by right translation) to study duality results for exotic crossed products. In [15] it is shown that any $G$-invariant weak*-closed ideal $E$ in the Fourier-Stieltjes algebra $B(G)$ of $G$ gives rise to exotic crossed-product norms $\|\cdot\|_{E}$ such that the crossed products $A \rtimes_{\alpha, E} G$ always admit dual coactions $\widehat{\alpha}_{E}$. Using such norms, the $A_{u}^{G, \alpha}-A \rtimes_{\alpha} G$ bimodule $\mathcal{F}_{u}(A)$ factors to give a partial equivalence $A_{E}^{G, \alpha}-A \rtimes_{\alpha, E} G$ bimodule $\mathcal{F}(A)_{E}$ and following ideas of Quigg in [24], we shall show that there exists a canonical coaction of $G$ on $\mathcal{F}(A)_{E}$ which is compatible with $\widehat{\alpha}_{E}$ and therefore implements a coaction $\delta_{E}$ on the E-generalized fixed-point algebra $A_{E}^{G, \alpha}$. The dual system for the coaction crossed product $\left(A_{E}^{G, \alpha} \rtimes_{\delta_{E}} \widehat{G}, \widehat{\delta}_{E}\right)$ is isomorphic to the original system $(A, \alpha)$ and the coaction $\delta_{E}$ on $B_{E}:=A_{E}^{G, \alpha}$ satisfies E-Katayama duality in the sense that

$$
B_{E} \rtimes_{\delta_{E}} G \rtimes_{\widehat{\delta}, E} G \cong B_{E} \otimes \mathcal{K}\left(L^{2} G\right)
$$

via a certain canonical map. (In fact a similar statement is true for any completion $A \rtimes_{\alpha, \mu} G$ of $C_{c}(G, A)$ by any $C^{*}$-norm $\|\cdot\|_{\mu}$ which admits a dual coaction $\widehat{\alpha}_{\mu}$. Such norms are not necessarily attached to an ideal $E$ in $B(G))$. Thus our results can be viewed as a version of Landstad duality for $E$-coactions. 
In particular, if we start with any coaction $\delta: B \rightarrow \mathcal{M}\left(B \otimes C^{*}(G)\right)$, then $A=B \rtimes_{\delta} \widehat{G}$ becomes a weakly proper $G \rtimes G$-algebra in a canonical way. Given $E \subseteq B(G)$ as above, the corresponding coaction $\delta_{E}$ on $B_{E}:=\left(B \rtimes_{\delta} \widehat{G}\right)_{E}^{G}, \widehat{\delta}$ has the following properties:

(E1) $\delta_{E}$ satisfies $E$-Katayama duality, i.e., $B_{E} \rtimes_{\delta_{E}} G \rtimes_{\widehat{\delta}, E} G \cong B_{E} \otimes \mathcal{K}\left(L^{2} G\right)$.

(E2) The dual systems $\left(B \rtimes_{\delta} \widehat{G}, \widehat{\delta}\right)$ and $\left(B_{E} \rtimes_{\delta_{E}} G, \widehat{\delta}_{E}\right)$ are isomorphic.

(E3) If $E=B(G)$, then $\left(B_{u}, \delta_{u}\right):=\left(B_{B(G)}, \delta_{B(G)}\right)$ is the maximalization of $(B, \delta)$ and if $E=B_{r}(G)$ (the weak*-closure of $\left.A(G)\right)$, then $\left(B_{r}, \delta_{r}\right):=$ $\left(B_{B_{r}(G)}, \delta_{B_{r}(G)}\right)$ is the normalization of $\delta$.

Note that for $E=B(G)$ we get maximal crossed products and for $E=B_{r}(G)$ we get reduced crossed products. For $\left(B_{u}, \delta_{u}\right)$ and $\left(B_{r}, \delta_{r}\right)$ being the maximalization and normalization of $\delta$ means in particular that the dual systems

$$
\left(B_{u} \rtimes_{\delta_{u}} \widehat{G}, \widehat{\delta}_{u}\right), \quad\left(B \rtimes_{\delta} \widehat{G}, \widehat{\delta}\right) \text { and }\left(B_{r} \rtimes_{\delta_{r}} \widehat{G}, \widehat{\delta}_{r}\right)
$$

coincide, $\left(B_{u}, \delta_{u}\right)$ satisfies duality for the full crossed product and $\left(B_{r}, \delta_{r}\right)$ satisfies duality for the reduced crossed products. Normal coactions have been introduced by Quigg in 25] and they are in natural one-to-one correspondence to coactions of the reduced group $\mathrm{C}^{*}$-algebra $C_{r}^{*}(G)$. It is also shown in 25] that every coaction has a normalization as above. A proof that maximalizations exist was first given in [10] (see [13 for a construction in case of quantum groups), but the construction given here is canonical and has better functorial properties. Indeed, in the two final sections of this paper we prove that our constructions of exotic (and, in particular, maximal and reduced) generalized fixed-point algebras are functorial for categories based on (equivariant) homomorphisms between $C^{*}$-algebras and extend to exotic norms the categorical version of Landstad duality obtained by Kaliszewski, Quigg and Raeburn in [18].

Furthermore, from the above results we also deduce a positive answer to 15 , Conjecture 6.14]: if $E$ is a $G$-invariant weak*-closed ideal in $B(G)$, then for any action $\alpha: G \rightarrow \operatorname{Aut}(A)$ the dual coaction $\widehat{\alpha}_{E}$ on $A \rtimes_{\alpha, E} G$ satisfies E-Katayama duality. On the other hand, we shall also give an example which shows that there are coactions which do not satisfy $E$-duality for any given $E \subseteq B(G)$ as above, thus giving a negative answer to [15, Conjecture 6.12].

\section{SOME PRELIMINARIES ON PROPER ACTIONS}

Recall that an action of a locally compact group $G$ on a locally compact (Hausdorff) space $X$ is called proper if the map

$$
G \times X \rightarrow X \times X ;(g, x) \mapsto(g x, x)
$$

is proper in the sense that inverse images of compact sets are compact. Proper actions are extremely nice: their orbit spaces $G \backslash X$ are always locally compact and Hausdorff and all stabilizers $G_{x}=\{g \in G: g x=x\}$ are compact. Another property which characterizes properness is the so-called wandering condition: for any two compact subsets $K_{1}, K_{2} \subseteq X$ the set $C\left(K_{1}, K_{2}\right):=\left\{g \in G: g K_{1} \cap K_{2} \neq \emptyset\right\}$ is compact in $G$. Let $\tau: G \rightarrow \operatorname{Aut}\left(C_{0}(X)\right)$ denote the corresponding action of $G$ on the algebra $C_{0}(X)$ of continuous functions on $X$ which vanish at $\infty$ given by

$$
\left(\tau_{g}(f)\right)(x)=f\left(g^{-1} \cdot x\right) .
$$

Let $C_{0}(X) \rtimes_{\tau} G$ denote the crossed product for the action of $G$ on $C_{0}(X)$ (note that for proper actions on spaces the full and reduced crossed products coincide, so we can take either construction here). If the action of $G$ on $X$ is proper and free (i.e., all stabilizers are trivial), it follows from work of Green and Rieffel (e.g. see [14,27]) that there is a canonical $C_{0}(G \backslash X)-C_{0}(X) \rtimes_{\tau} G$ Morita equivalence constructed 
as follows: Let $B_{0}=C_{c}\left(G, C_{0}(X)\right)$ be viewed as a dense subalgebra of $C_{0}(X) \rtimes_{\tau} G$ and let $E_{0}=C_{c}(G \backslash X) \subseteq C_{0}(G \backslash X)$. Then $\mathcal{F}_{c}(X):=C_{c}(X)$ can be made into an $E_{0}-B_{0}$ pre-imprimitivity bimodule by defining left and right $E_{0^{-}}$and $B_{0}$-valued inner products and left and right actions of $E_{0}$ and $B_{0}$ on $\mathcal{F}_{c}(X)$, respectively, given by

$$
\begin{aligned}
& \langle\langle\xi \mid \eta\rangle\rangle_{B_{0}}(t, x)=\Delta(t)^{-1 / 2} \overline{\xi(x)} \eta\left(t^{-1} x\right) \\
& E_{0}\langle\langle\xi \mid \eta\rangle\rangle(G x)=\int_{G} \eta\left(t^{-1} x\right) \overline{\xi\left(t^{-1} x\right)} d t \\
& \left.(\xi \cdot \varphi)(x)=\int_{G} \Delta(t)^{-1 / 2} \xi\left(t^{-1} x\right) \varphi\left(t^{-1}, t^{-1} x\right)\right) d t, \quad \text { and } \\
& f \cdot \xi(x)=f(G x) \xi(x)
\end{aligned}
$$

for all $\xi, \eta \in \mathcal{F}_{c}(X), \varphi \in B_{0}$ and $f \in E_{0}$. The pre-imprimitivity bimodule $\mathcal{F}_{c}(X)$ completes to give a $C_{0}(G \backslash X)-C_{0}(X) \rtimes_{\tau} G$ imprimitivity bimodule $\mathcal{F}(X)$. If the action of $G$ is not free, we still get a Hilbert $C_{0}(G \backslash X)-C_{0}(X) \rtimes_{\tau} G$-bimodule $\mathcal{F}(X)$, but the $C_{0}(X) \rtimes_{\tau} G$-valued inner product will not be full. But if we define

$$
\left.I_{X}:=\overline{\operatorname{span}}\{\langle\xi \mid \eta\rangle\rangle_{C_{0}(X) \rtimes G}: \xi, \eta \in \mathcal{F}(X)\right\},
$$

then $I_{X}$ is a closed ideal in $C_{0}(X) \rtimes G$ such that $\mathcal{F}(X)$ becomes a $C_{0}(G \backslash X)-I_{X}$ imprimitivity bimodule.

There have been many attempts to extend the notion of properness to $C^{*}$-dynamical systems $(A, G, \alpha)$ and to obtain analogues of the above Morita equivalences. The weakest notion for proper actions is due to Rieffel (see [28, 29]), and his concept has been studied in a number of papers by several authors (e.g. see [2, 4, 5, 18, 23, 28, 29]). In [28] Rieffel says that an action $\alpha: G \rightarrow \operatorname{Aut}(A)$ is proper, if there exists a dense $\alpha$-invariant subalgebra $A_{c}$ (playing the role of $C_{c}(X)$ ) such that the following conditions are satisfied:

- For all $a, b \in A_{c}$ the functions $t \mapsto \Delta(t)^{-1 / 2} a^{*} \alpha_{t}(b)$ and $t \mapsto a^{*} \alpha_{t}(b)$ lie in $L^{1}(G, A)$, and

- for any $a, b \in A_{c}$ there is a unique element $E_{0}\langle\langle a \mid b\rangle\rangle$ in

$$
\mathcal{M}\left(A_{c}\right)^{G, \alpha}=\left\{m \in \mathcal{M}(A)^{G, \alpha}: m A_{c}, A_{c} m \subseteq A_{c}\right\}
$$

such that for all $c \in A_{c}$ we have

$$
E_{0}\langle\langle a \mid b\rangle\rangle c=\int_{G} \alpha_{t}\left(a b^{*}\right) c d t .
$$

Notice that, different from [28, we let $E_{0}\langle\langle a \mid b\rangle\rangle$ act on the left of $A_{c}$. Thus our constructions are dual to the ones performed by Rieffel. A similar approach which is consistent with our constructions (up to factors involving modular functions) has been used by Meyer in 23 .

Under the above conditions, Rieffel shows that $A_{c}$ equipped with the $A \rtimes_{\alpha, r} G$ valued inner product

$$
\langle\langle a \mid b\rangle\rangle_{A \rtimes_{r} G}=\left(t \mapsto \Delta(t)^{-1 / 2} a^{*} \alpha_{t}(b)\right) \in L^{1}(G, A) \subseteq A \rtimes_{r} G
$$

completes to give a Hilbert $A \rtimes_{\alpha, r} G$-module $\mathcal{F}(A)_{r}$ such that its $C^{*}$-algebra of compact operators can be naturally identified with the generalized fixed-point algebra $A^{G, \alpha}$ defined as

$$
A^{G, \alpha}:=\overline{\operatorname{span}}\left\{E_{0}\langle\langle a \mid b\rangle\rangle: a, b \in A_{c}\right\} \subseteq \mathcal{M}(A) .
$$

A proper action in this sense is called saturated if the $A \rtimes_{\alpha, r} G$-valued inner product on $\mathcal{F}(A)_{r}$ is full, so that $\mathcal{F}(A)_{r}$ becomes a $A^{G, \alpha}-A \rtimes_{\alpha, r} G$ imprimitivity bimodule. In general, $\mathcal{F}(A)_{r}$ will be an imprimitivity bimodule between $A^{G, \alpha}$ and the ideal $I(A)_{r}:=\overline{\operatorname{span}}\left\{\langle\langle a \mid b\rangle\rangle_{A \rtimes_{r} G}: a, b \in A_{c}\right\}$. The notion of integrability introduced in 
29 even extends the above notion of properness, but lacks a suitable definition of generalized fixed-point algebras and corresponding Morita equivalences. Another extension of Rieffel's theory is given by the theory of continuously square-integrable actions due to Meyer (see [23]), which seems to be the weakest notion of properness allowing a construction of a reduced generalized fixed-point algebra. However it is not clear whether such proper actions allow the construction of full generalized fixed-point algebras.

Assume now that $A$ is a weakly proper $X \rtimes G$-algebra. Recall that this means that $X$ is a proper $G$-space and that $A$ is endowed with an action $\alpha: G \rightarrow \operatorname{Aut}(A)$ and a $G$-equivariant nondegenerate *-homomorphism $\phi: C_{0}(X) \rightarrow \mathcal{M}(A)$. We shall sometimes simply say that $(A, \alpha)$ is a weak $X \rtimes G$-algebra. It has been shown by Rieffel in [29, Propositon 5.7] that $A_{c}:=\phi\left(C_{c}(X)\right) A \phi\left(C_{c}(X)\right)$ provides a dense subalgebra as in the above discussion and therefore the action of $G$ on $A$ is proper in Rieffel's sense.

To see that the notion of weakly proper $X \rtimes G$-algebras is quite general we should remark that every action $\alpha: G \rightarrow \operatorname{Aut}(A)$ is Morita equivalent to a weakly proper $G \rtimes G$-action (with $G$ acting on itself by right translation): simply consider the action $\alpha \otimes \operatorname{Ad} \rho: G \rightarrow A \otimes \mathcal{K}\left(L^{2} G\right)$, where $\rho$ denotes the right regular representation, and then $1_{A} \otimes M: C_{0}(G) \rightarrow \mathcal{M}\left(A \otimes \mathcal{K}\left(L^{2} G\right)\right)$ provides the desired $G$-equivariant homomorphism, where $M: C_{0}(G) \rightarrow \mathcal{L}\left(L^{2} G\right)$ denotes the representation by multiplication operators. We should also note that weakly proper $X \rtimes G$-algebras have been studied in several papers (using different terminology) in the context of Rieffel-proper actions (e.g. see [2,3,18,).

In what follows, we often write $f \cdot a$ (resp. $a \cdot f$ ) for the element $\phi(f) a$ (resp. $a \phi(f))$ if $a \in \mathcal{M}(A)$ and $f \in C_{b}(X)$.

Definition 2.1. Suppose that $A$ is a weakly proper $X \rtimes G$-algebra. Let $A_{c}:=$ $C_{c}(X) \cdot A \cdot C_{c}(X)$ and let

$$
\widetilde{A}_{c}^{G, \alpha}:=\left\{m \in \mathcal{M}(A)^{G, \alpha}: m \cdot f, f \cdot m \in A_{c} \text { for all } f \in C_{c}(X)\right\} .
$$

Then the generalized fixed-point algebra for $A$ with compact supports is the algebra

$$
A_{c}^{G, \alpha}:=C_{c}(G \backslash X) \cdot \widetilde{A}_{c}^{G, \alpha} \cdot C_{c}(G \backslash X) .
$$

It is straightforward to check that $A_{c}^{G, \alpha}$ is a ${ }^{*}$-subalgebra of $\mathcal{M}(A)$. In the following proposition we let $\|\cdot\|_{\mu}$ denote any crossed-product norm, meaning any given $C^{*}$-norm on $C_{c}(G, A)$ such that $\|\cdot\|_{r} \leq\|\cdot\|_{\mu} \leq\|\cdot\|_{u}$, where $\|\cdot\|_{r}$ denotes the reduced norm given by the (left) regular representation $\Lambda_{A}$ of $C_{c}(G, A)$ on $L^{2}(G, A)$ and $\|\cdot\|_{u}$ denotes the universal (or maximal) norm on $C_{c}(G, A)$. We then write $A \rtimes_{\alpha, \mu} G$ for the completion of $C_{c}(G, A)$ with respect to $\|\cdot\|_{\mu}$ and call $A \rtimes_{\alpha, \mu} G$ the $\mu$-crossed product of $(A, G, \alpha)$. Observe that such exotic crossed products correspond to quotients of $A \rtimes_{\alpha, u} G$ by ideals contained in $\operatorname{ker}\left(\Lambda_{A}\right)$. With this notation we get:

Proposition 2.2. Suppose that $A$ is a weakly proper $X \rtimes G$-algebra. Let $\mathcal{F}_{c}(A)=$ $C_{c}(X) \cdot A 1$ and let $B_{0}=C_{c}(G, A)$ be viewed as a dense subalgebra of $A \rtimes_{\alpha, \mu} G$. Then there is a $B_{0}$-valued inner product on $\mathcal{F}_{c}(A)$ and a right action of $B_{0}$ on $\mathcal{F}_{c}(A)$ defined as

$$
\begin{aligned}
\langle\langle\xi \mid \eta\rangle\rangle_{B_{0}}(t) & =\Delta(t)^{-1 / 2} \xi^{*} \alpha_{t}(\eta) \quad \text { and } \\
\xi \cdot \varphi & =\int_{G} \Delta(t)^{-1 / 2} \alpha_{t}\left(\xi \varphi\left(t^{-1}\right)\right) d t
\end{aligned}
$$

\footnotetext{
${ }^{1}$ Note that $\mathcal{F}_{c}(A)$ is a vector space, since if $a=\sum_{k=1}^{l} f_{i} \cdot a_{i}$ is a linear combination, then $a=f \cdot a$ for any $f \in C_{c}(X)$ such that $f \equiv 1$ on $\cup\left\{\operatorname{supp}\left(f_{i}\right): 1 \leq k \leq l\right\}$.
} 
which turns $\mathcal{F}_{c}(A)$ into a pre-Hilbert $B_{0}$-module. These operations extend to the completion $\mathcal{F}_{\mu}(A)$ with respect to the norm $\|\xi\|_{\mu}:=\left\|\langle\xi \mid \eta\rangle_{B_{0}}\right\|_{\mu}^{1 / 2}$, so that $\mathcal{F}_{\mu}(A)$ becomes a Hilbert $A \rtimes_{\alpha, \mu} G$-module. Moreover, there is a faithful ${ }^{*}$-homomorphism $\Psi_{\mu}: A_{c}^{G, \alpha} \rightarrow \mathcal{K}\left(\mathcal{F}_{\mu}(A)\right)$ with dense image, given by

$$
\Psi_{\mu}(m) \xi=m \xi \quad \text { for all } m \in A_{c}^{G, \alpha}, \xi \in \mathcal{F}_{c}(A),
$$

where on the right hand side we use multiplication inside $\mathcal{M}(A)$.

Before we start with the proof, we want to give a definition of an $A_{c}^{G, \alpha}$-valued inner product on $\mathcal{F}_{c}(A)$ which will play a crucial role in the proof of the proposition. Recall that $A_{c}:=C_{c}(X) \cdot A \cdot C_{c}(X)$. The following lemma is basically due to Rieffel and Kaliszewski-Quigg-Raeburn (see the proof of [29, Theorem 5.7] and [18, §2]). Recall first that for any proper $G$-space $X$, there exists a surjective linear map

$$
\mathbb{E}_{\tau}: C_{c}(X) \rightarrow C_{c}(G \backslash X) ; \quad \mathbb{E}_{\tau}(f)(G x)=\int_{G} f\left(t^{-1} x\right) d t
$$

This extends to weakly proper $X \rtimes G$-algebras $A$ as follows:

Lemma 2.5. There is well-defined surjective linear map $\mathbb{E}: A_{c} \rightarrow A_{c}^{G, \alpha}$ such that

$$
\mathbb{E}(a) c=\int_{G} \alpha_{t}(a) c d t \quad \text { for all } c \in A_{c} .
$$

The map $\mathbb{E}$ has the following properties:

(1) We have $\mathbb{E}\left(a^{*}\right)=\mathbb{E}(a)^{*}, \mathbb{E}(\psi \cdot a)=\psi \cdot \mathbb{E}(a)$ and $\mathbb{E}(a \cdot \psi)=\mathbb{E}(a) \cdot \psi$ for all $\psi \in C_{c}(G \backslash X)$ and $a \in A_{c}$.

(2) For all $m \in \tilde{A}_{c}^{G, \alpha}, a \in A_{c}$ and $f \in C_{c}(X)$ we have $\mathbb{E}(m a)=m \mathbb{E}(a)$, $\mathbb{E}(m \cdot f)=m \cdot \mathbb{E}_{\tau}(f), \mathbb{E}(a m)=\mathbb{E}(a) m$ and $\mathbb{E}(f \cdot m)=\mathbb{E}_{\tau}(f) \cdot m$.

(3) For every $m \in A_{c}^{G, \alpha}$ there exists $f \in C_{c}(X)$ such that $\mathbb{E}(m \cdot f)=\mathbb{E}(f \cdot m)=$ $m$.

(4) For all pairs $f, g \in C_{c}(X)$ the linear map $\mathbb{E}_{f, g}: A \rightarrow \mathcal{M}(A) ; a \mapsto \mathbb{E}(f \cdot a \cdot g)$ is norm continuous.

Moreover, there is an $A_{c}^{G, \alpha}$-valued inner product on $\mathcal{F}_{c}(A)$ given by

$$
A_{c}^{\alpha}\langle\langle\xi \mid \eta\rangle\rangle:=\mathbb{E}\left(\xi \eta^{*}\right)
$$

which then satisfies the equation

$$
\xi \cdot\langle\langle\eta \mid \zeta\rangle\rangle_{B_{0}}={ }_{A_{c}^{\alpha}}\langle\langle\xi \mid \eta\rangle\rangle \cdot \zeta
$$

for all $\xi, \eta, \zeta \in \mathcal{F}_{c}(A)$, where the left and right actions and the $B_{0}$-valued inner products are as in the proposition.

Proof. The fact that formula (2.6) determines a unique element in $\mathcal{M}(A)^{G, \alpha}$ has been shown in the proof of [29, Proposition 5.7] and it follows easily from this formula that $\mathbb{E}\left(a^{*}\right)=\mathbb{E}(a)^{*}$ for all $a \in A_{c}$. The formulas in (1) and (2) are easy to check but can also be found in [18, Lemma 2.1 and Lemma 2.3].

It is shown in [18, Lemma 2.3] that $f \cdot \mathbb{E}(a), \mathbb{E}(a) \cdot f \in A_{c}$. So $\mathbb{E}(a) \in \tilde{A}_{c}^{G, \alpha}$. Now if $a=f \cdot b \cdot g$ for some $f, g \in C_{c}(X)$ and $b \in A$ and if $\psi \in C_{c}(G \backslash X)^{+}$such that $\psi \equiv 1$ on $G \cdot(\operatorname{supp} f \cup \operatorname{supp} g)$, then $a=\psi \cdot a \cdot \psi$ and (1) implies

$$
\mathbb{E}(a)=\mathbb{E}(\psi \cdot a \cdot \psi)=\psi \cdot \mathbb{E}(a) \cdot \psi
$$

from which it follows that $\mathbb{E}$ takes values in the algebra $A_{c}^{G, \alpha}$. On the other hand, if $m \in A_{c}^{G, \alpha}$, we can write $m=\psi \cdot m \cdot \psi$ for some function $\psi \in C_{c}(G \backslash X)$. Now, choose a function $f \in C_{c}(X)$ such that $\mathbb{E}_{\tau}(f)=\psi$. Then it follows from (2) that $\mathbb{E}(m \cdot f)=m \cdot \mathbb{E}_{\tau}(f)=m$ and similarly that $\mathbb{E}(f \cdot m)=m$. Since $m \cdot f \in A_{c}$, this shows in particular that $\mathbb{E}\left(A_{c}\right)=A_{c}^{G, \alpha}$.

After having observed surjectivity of $\mathbb{E}: A_{c} \rightarrow A_{c}^{G, \alpha}$ the properties for the $A_{c}^{G, \alpha_{-}}$ valued inner product follow from the proof of [29, Proposition 5.7]. 
Finally, (4) follows from [24, Lemma 3.6].

The map $\mathbb{E}: A_{c} \rightarrow A_{c}^{G, \alpha}$ will be sometimes written as an integral $\mathbb{E}(a)=$ $\int_{G}^{\text {st }} \alpha_{t}(a) d t$. It can be shown that $\mathbb{E}(a)$ is, indeed, an integral in the sense of Pettis with respect to the strict topology in $\mathcal{M}(A)$ ( $c f$. [7, Proposition 2.2]).

Proof of Proposition [2.2. Recall from [26. Lemma 2.16] that $\mathcal{F}_{c}(A)$ is a pre-Hilbert $B_{0}$-module if the right action of $B_{0}$ on $\mathcal{F}_{c}(A)$ and the $B_{0}$-valued inner product satisfy the following conditions:

- $\langle\langle\cdot \mid \cdot\rangle\rangle_{B_{0}}$ is $B_{0}$-linear in the second variable,

- $\langle\langle\xi \mid \eta\rangle\rangle_{B_{0}}^{*}=\langle\langle\eta \mid \xi\rangle\rangle_{B_{0}}$, and

- $\langle\langle\xi \mid \xi\rangle\rangle_{B_{0}} \geq 0$ as an element of $A \rtimes_{\alpha, \mu} G$ for all $\xi, \eta \in \mathcal{F}_{c}(A)$.

From these conditions the first two are well known (and easy to check), so we concentrate on the positivity condition. Since $A \rtimes_{\alpha, \mu} G$ is a quotient of the universal crossed product $A \rtimes_{\alpha} G=A \rtimes_{\alpha, u} G$, it suffices to show positivity in this case.

For this let $\xi \in \mathcal{F}_{c}(A)$ be given. Then we may write $\xi=f \cdot a$ for some $f \in C_{c}(X)$ and $a \in A$. Recall that the canonical embedding $\iota_{A}: A \rightarrow \mathcal{M}\left(A \rtimes_{\alpha} G\right)$ is determined by the equations

$$
\left(\iota_{A}(a) \varphi\right)(t)=a \varphi(t) \quad \text { and } \quad\left(\varphi \iota_{A}(a)\right)(t)=\varphi(t) \alpha_{t}(a) \quad \text { for } \varphi \in B_{0} .
$$

On the other hand, the $G$-equivariant *-homomorphism $\phi: C_{0}(X) \rightarrow \mathcal{M}(A)$ induces a *-homomorphism $\phi \rtimes G: C_{0}(X) \rtimes_{\tau} G \rightarrow \mathcal{M}\left(A \rtimes_{\alpha} G\right)$ such that, for a function $g \in C_{c}\left(G, C_{0}(X)\right)$ and $h \in C_{c}(G, A)$, we have $\phi \rtimes G(g) h=g * h$, where $g * h(t):=$ $\int_{G} g(s) \cdot \alpha_{s}\left(h\left(s^{-1} t\right)\right) d s$. Using this one easily checks that

$$
\left(\iota_{A}(a)(\phi \rtimes G)(g)\right)(t)=a \cdot g(t) \quad \text { and } \quad\left((\phi \rtimes G)(g) \iota_{A}(a)\right)(t)=g(t) \cdot \alpha_{t}(a)
$$

for all $g \in C_{c}\left(G, C_{0}(X)\right)$ and $a \in A$. We then compute

$$
\begin{aligned}
\langle\langle f \cdot a \mid f \cdot a\rangle\rangle_{B_{0}}(t) & =\Delta(t)^{-1 / 2}(f \cdot a)^{*} \alpha_{t}(f \cdot a)=a^{*} \cdot\left(\Delta(t)^{-1 / 2} \cdot\left(\bar{f} \tau_{t}(f)\right)\right) \cdot \alpha_{t}(a) \\
& \left.=\left(i_{a}\left(a^{*}\right)(\phi \rtimes G)(\langle f \mid f\rangle\rangle_{C_{0}(X) \rtimes G}\right) \iota_{A}(a)\right)(t) .
\end{aligned}
$$

Hence, we get

$$
\langle\langle\xi \mid \xi\rangle\rangle_{B_{0}}=\langle\langle f \cdot a \mid f \cdot a\rangle\rangle_{B_{0}}=\iota_{A}\left(a^{*}\right)(\phi \rtimes G)\left(\langle\langle f \mid f\rangle\rangle_{\left.C_{0}(X) \rtimes G\right) \iota_{A}(a)}\right.
$$

inside $\mathcal{M}\left(A \rtimes_{\alpha} G\right)$. Since the $C_{0}(X) \rtimes G$-valued inner product on $\mathcal{F}(X)=\overline{C_{c}(X)}$ is known to be positive, it follows that $\langle\langle f \cdot a \mid f \cdot a\rangle\rangle_{B_{0}}$ is positive in $A \rtimes_{\alpha} G$.

At this point we should also note that $\langle\langle\xi \mid \xi\rangle\rangle_{B_{0}} \neq 0$ if $\xi \neq 0$, which follows from the fact that $\langle\langle\xi \mid \xi\rangle\rangle_{B_{0}}(e)=\xi^{*} \xi>0$ in $A$ and that the imbedding of $C_{c}(G, A)$ into $A \rtimes_{\alpha, \mu} G$ is injective (since this is already true for the reduced crossed product).

It now follows that $\mathcal{F}_{c}(A)$ completes to give a Hilbert $A \rtimes_{\alpha, \mu} G$-module $\mathcal{F}_{\mu}(A)$. The fact that $A_{c}^{G, \alpha}$ acts as a dense subalgebra of $\mathcal{K}\left(\mathcal{F}_{\mu}(A)\right)$ follows from Lemma 2.5. Equation (2.7) implies that for any pair $\xi, \eta \in \mathcal{F}_{c}(A)$ the left inner product $A_{c}^{\alpha}\langle\langle\xi \mid \eta\rangle\rangle=\mathbb{E}\left(\xi \eta^{*}\right)$ acts as the compact operator $\Theta_{\xi, \eta}$ on $\mathcal{F}_{\mu}(A)$. In particular, this operator is non-zero if $\xi, \eta \neq 0$. Since $\mathcal{F}_{c}(A) \mathcal{F}_{c}(A)^{*}=A_{c}$ and since by Lemma 2.5 we have $\mathbb{E}\left(A_{c}\right)=A_{c}^{G, \alpha}$ we see that left multiplication of $A_{c}^{G, \alpha}$ on $\mathcal{F}_{c}(A)$ induces an injective *-homomorphism of $A_{c}^{G, \alpha}$ onto a dense subalgebra of $\mathcal{K}\left(\mathcal{F}_{\mu}(A)\right)$.

Definition 2.8. Suppose that $A$ is a weakly proper $X \rtimes G$-algebra and let $\|\cdot\|_{\mu}$ be any crossed-product norm on $C_{c}(G, A)$. Then the $\mu$-fixed-point algebra of $A$ is defined as

$$
A_{\mu}^{G, \alpha}:=\mathcal{K}\left(\mathcal{F}_{\mu}(A)\right)
$$

In other words, $A_{\mu}^{G, \alpha}$ is the completion of $A_{c}^{G, \alpha}$ with respect to the norm induced by the left action of $A_{c}^{G, \alpha}$ on $\mathcal{F}_{\mu}(A)$. 
We now want to give an alternative construction of the module $\mathcal{F}_{\mu}(A)$. For this recall that $\mathcal{F}(X)=\mathcal{F}\left(C_{0}(X)\right)$ denotes the canonical Hilbert $C_{0}(G \backslash X)-C_{0}(X) \rtimes_{\tau} G$ bimodule for the proper $G$-space $X$. To make notations a bit more convenient, we shall write from now on $\langle\langle f \mid g\rangle\rangle_{X}$ instead of $\left\langle\langle f \mid g\rangle_{C_{0}(X) \rtimes_{\tau} G}\right.$ for the $C_{0}(X) \rtimes_{\tau} G$ valued inner product on $\mathcal{F}(X)$.

Proposition 2.9. Suppose that $A$ is a weakly proper $X \rtimes G$-algebra. Let $\phi \rtimes_{\mu} G$ : $C_{0}(X) \rtimes_{\tau} G \rightarrow \mathcal{M}\left(A \rtimes_{\alpha, \mu} G\right)$ denote the canonical nondegenerate ${ }^{*}$-homomorphism induced from the $G$-equivariant ${ }^{*}$-homomorphism $\phi: C_{0}(X) \rightarrow \mathcal{M}(A)$. Then there is an isomorphism of Hilbert $A \rtimes_{\alpha, \mu} G$-modules

$$
\Psi_{\mu}: \mathcal{F}(X) \otimes_{C_{0}(X) \rtimes G}\left(A \rtimes_{\alpha, \mu} G\right) \rightarrow \mathcal{F}_{\mu}(A)
$$

given on elementary tensors $f \otimes \varphi \in C_{c}(X) \otimes C_{c}(G, A)$ by

$$
\Psi_{\mu}(f \otimes \varphi)=\int_{G} \Delta(t)^{-1 / 2} \alpha_{t}\left(f \cdot \varphi\left(t^{-1}\right)\right) d t .
$$

In particular, it follows that the $A \rtimes_{\alpha, \mu}$ G-valued inner product on $\mathcal{F}_{\mu}(A)$ is full whenever $G$ acts freely on $X$.

Proof. An easy computation shows that $\Psi_{\mu}(f \otimes \varphi) \cdot \psi=\Psi_{\mu}(f \otimes(\varphi * \psi))$ for all $\varphi, \psi \in C_{c}(G, A)$ and $f \in C_{c}(X)$, so that the result will follow if we can show that $\Psi_{\mu}$ is an isometry with dense image in $\mathcal{F}_{\mu}(A)$. To see that it is isometric it suffices to check $\left\langle\left\langle\Psi_{\mu}(f \otimes \varphi) \mid \Psi_{\mu}(g \otimes \psi)\right\rangle\right\rangle_{B_{0}}=\langle\langle f \otimes \varphi \mid g \otimes \psi\rangle\rangle_{B_{0}}$ for all $f \otimes \varphi, g \otimes \psi \epsilon$ $C_{c}(X) \otimes C_{c}(G, A)$. For this we compute

$$
\begin{aligned}
& \langle\langle f \otimes \varphi \mid g \otimes \psi\rangle\rangle_{B_{0}}(t)=\left\langle\left\langle\varphi \mid\langle\langle f \mid g\rangle\rangle_{X} \cdot \psi\right\rangle\right\rangle_{B_{0}}(t) \\
& =\varphi^{*} *\left(\langle\langle f \mid g\rangle\rangle_{X} * \psi\right)(t) \\
& =\int_{G} \int_{G} \Delta\left(s^{-1}\right) \alpha_{s}\left(\varphi\left(s^{-1}\right)^{*} \Delta(r)^{-1 / 2} \bar{f} \tau_{r}(g) \alpha_{r}\left(\psi\left(r^{-1} s^{-1} t\right)\right)\right) d r d s \\
& \stackrel{r \mapsto s^{-1}}{=} \operatorname{tr} \int_{G} \int_{G} \Delta(s t r)^{-1 / 2} \alpha_{s}\left(\varphi\left(s^{-1}\right)^{*} \bar{f} \tau_{s^{-1} t r}(g) \alpha_{s^{-1} t r}\left(\psi\left(r^{-1}\right)\right)\right) d r d s \\
& =\int_{G} \int_{G} \Delta(s t r)^{-1 / 2} \alpha_{s}\left(\varphi\left(s^{-1}\right)\right)^{*} \tau_{s}(\bar{f}) \tau_{t r}(g) \alpha_{t r}\left(\psi\left(r^{-1}\right)\right) d r d s \\
& =\Delta(t)^{-1 / 2} \Psi_{\mu}(f \otimes \varphi)^{*} \alpha_{t}\left(\Psi_{\mu}(g \otimes \psi)\right) \\
& =\left\langle\left\langle\Psi_{\mu}(f \otimes \varphi) \mid \Psi_{\mu}(g \otimes \psi)\right\rangle\right\rangle_{B_{0}}(t) .
\end{aligned}
$$

Thus the proposition will follow if we can show that $\Psi_{\mu}$ has dense image in $\mathcal{F}_{\mu}(A)$. We postpone the proof for this to Lemma 2.13 below.

In what follows we need to argue at several instances with certain inductive limit topologies on $B_{0}=C_{c}(G, A), A_{c}=C_{c}(X) \cdot A \cdot C_{c}(X), \mathcal{F}_{c}(A)=C_{c}(X) \cdot A$ and $A_{c}^{G, \alpha}$. In case of $B_{0}=C_{c}(G, A)$, this is the usual definition of uniform convergence of nets $\left(\varphi_{i}\right)$ with supports $\operatorname{supp} \varphi_{i}$ lying in a fixed compact set for all $i \in I$. In the other cases the definitions are as follows:

Definition 2.11. (1) If $\left(a_{i}\right)_{i \in I}$ is a net in $\mathcal{F}_{c}(A)$ (resp. in $A_{c}$ ), we say that $a_{i} \rightarrow a \in \mathcal{F}_{c}(A)$ (resp. in $A_{c}$ ) in the inductive limit topology, if $a_{i} \rightarrow a$ in $A$ in the norm topology and there exists some $f \in C_{c}(X)$ such that $a_{i}=f \cdot a_{i}$ (resp. $\left.a_{i}=f \cdot a_{i} \cdot f\right)$ for all $i \in I$.

(2) If $\left(m_{i}\right)_{i \in I}$ is a net in $A_{c}^{G, \alpha}$, we say that $m_{i} \rightarrow m \in A_{c}^{G, \alpha}$ in the inductive limit topology if $m_{i} \rightarrow m$ in $\mathcal{M}(A)$ in norm and the following conditions hold:

(i) There exists $\psi \in C_{c}(G \backslash X)$ such that $m_{i}=\psi \cdot m_{i} \cdot \psi$ for all $i \in I$, and

(ii) $m_{i} \cdot f \rightarrow m \cdot f$ and $f \cdot m_{i} \rightarrow f \cdot m$ in the inductive limit topology of $A_{c}$ for all $f \in C_{c}(X)$. 
Lemma 2.12. Let $A$ be a weakly proper $X \rtimes G$-algebra. Then all pairings in the pre-Hilbert $A_{c}^{G, \alpha}-B_{0}$-bimodule $\mathcal{F}_{c}(A)$ are jointly continuous with respect to the respective inductive limit topologies.

Proof. There are four pairings to consider: the $A_{c}^{G, \alpha_{-}}$and $B_{0}$-valued inner products on $\mathcal{F}_{c}(A)$ and the pairings coming from the left and right actions of $A_{c}^{G, \alpha}$ and $B_{0}$ on $\mathcal{F}_{c}(A)$, respectively. We do the inner products here and leave the action-pairings to the reader.

For the $B_{0}$-valued inner product on $\mathcal{F}_{c}(A)$, let $\xi_{i} \rightarrow \xi$ and $\eta_{i} \rightarrow \eta$ in the inductive limit topology of $\mathcal{F}_{c}(A)$ and let $f \in C_{c}(X)$ such that $f \cdot \xi_{i}=\xi_{i}$ and $f \cdot \eta_{i}=\eta_{i}$ for all $i \in I$. Then the computations in the proof of Proposition 2.2 show that

$$
\left\langle\left\langle\xi_{i} \mid \eta_{i}\right\rangle\right\rangle_{B_{0}}(t)=\left\langle\left\langle f \cdot \xi_{i} \mid f \cdot \eta_{i}\right\rangle\right\rangle_{B_{0}}(t)=\xi_{i} \cdot\left\langle\langle f \mid f\rangle_{X}(t) \cdot \alpha_{t}\left(\eta_{i}\right)\right.
$$

which clearly converges to $t \mapsto \xi \cdot\langle\langle f \mid f\rangle\rangle_{X}(t) \cdot \alpha_{t}(\eta)=\langle\langle\xi \mid \eta\rangle\rangle_{B_{0}}(t)$ in the inductive limit topology of $B_{0}$.

For the left inner product $A_{c}^{\alpha}\langle\langle\cdot \mid \cdot\rangle\rangle$ we first observe that if $\xi_{i} \rightarrow \xi$ and $\eta_{i} \rightarrow \eta$ in the inductive limit topology of $\mathcal{F}_{c}(A)$, then $\xi_{i} \eta_{i}^{*} \rightarrow \xi \eta^{*}$ in the inductive limit topology of $A_{c}$ and it follows then from Lemma 2.5 (4) that $A_{c}^{\alpha}\left\langle\left\langle\xi_{i} \mid \eta_{i}\right\rangle\right\rangle=\mathbb{E}\left(\xi_{i} \eta_{i}^{*}\right) \rightarrow$ $\mathbb{E}\left(\xi \eta^{*}\right)={ }_{A_{c}^{\alpha}}\langle\langle\xi \mid \eta\rangle\rangle$ in norm. Moreover, if $f \in C_{c}(X)$ such that $f \cdot\left(\xi_{i} \eta_{i}^{*}\right) \cdot f=\xi_{i} \eta_{i}^{*}$ for all $i \in I$ and if $\psi \in C_{c}(G \backslash X)$ such that $\psi \equiv 1$ on $G \cdot \operatorname{supp} f$, then it follows that $\psi \cdot{ }_{A_{c}^{\alpha}}\left\langle\left\langle\xi_{i} \mid \eta_{i}\right\rangle\right\rangle \cdot \psi={ }_{A_{c}^{\alpha}}\left\langle\left\langle\xi_{i} \mid \eta_{i}\right\rangle\right\rangle$ for all $i \in I$. Finally, for fixed $g \in C_{c}(X)$, we have

$$
\begin{aligned}
g \cdot A_{c}^{\alpha}\left\langle\left\langle\xi_{i} \mid \eta_{i}\right\rangle\right\rangle & =g \cdot \mathbb{E}\left(f \cdot \xi_{i} \eta_{i}^{*} \cdot f\right) \\
& =\int_{G}^{\mathrm{st}} g \cdot \alpha_{t}\left(f \cdot \xi_{i} \eta_{i}^{*} \cdot f\right) d t \\
& =\int_{G}^{\mathrm{st}} g \cdot \tau_{t}(f) \cdot \alpha_{t}\left(\xi_{i} \eta_{i}^{*}\right) \cdot \tau_{t}(f) d t .
\end{aligned}
$$

If we choose a function $h \in C_{c}(X)$ such that $h \equiv 1$ on $K \cdot \operatorname{supp} f \cup \operatorname{supp} g$, where $\left.K=\operatorname{supp}\left(t \mapsto g \tau_{t}(f)\right)\right) \subseteq G$, we see that $h \cdot\left(g \cdot A_{c}^{\alpha}\left\langle\left\langle\xi_{i} \mid \eta_{i}\right\rangle\right\rangle\right) \cdot h=g \cdot A_{c}^{\alpha}\left\langle\left\langle\xi_{i} \mid \eta_{i}\right\rangle\right\rangle$ for all $i \in I$ and it is easy to check from the above formulas that $g \cdot A_{c}^{\alpha}\left\langle\left\langle\xi_{i} \mid \eta_{i}\right\rangle\right\rangle \rightarrow g \cdot A_{c}^{\alpha}\langle\langle\xi \mid \eta\rangle\rangle$ in norm. Hence $g \cdot A_{c}^{\alpha}\left\langle\left\langle\xi_{i} \mid \eta_{i}\right\rangle\right\rangle \rightarrow g \cdot A_{c}^{\alpha}\langle\langle\xi \mid \eta\rangle\rangle$ in the inductive limit topology and a similar argument shows that $A_{c}^{\alpha}\left\langle\left\langle\xi_{i} \mid \eta_{i}\right\rangle\right\rangle \cdot g \rightarrow A_{c}^{\alpha}\left\langle\left\langle\xi_{i} \mid \eta_{i}\right\rangle\right\rangle \cdot g$ in the inductive limit topology of $A_{c}$.

Lemma 2.13. Let $\xi=f \cdot a \in \mathcal{F}_{c}(A)$ be fixed. Then, there exists a net $\varphi_{i} \in C_{c}(G, A)$ such that $\Psi_{\mu}\left(f \otimes \varphi_{i}\right)=\int_{G} \Delta(t)^{-1 / 2} \alpha_{t}\left(f \cdot \varphi_{i}\left(t^{-1}\right)\right) d t$ converges to $\xi$ in the inductive limit topology of $\mathcal{F}_{c}(A)$. In particular, the map $\Psi_{\mu}: \mathcal{F}(X) \otimes_{C_{0}(X) \rtimes G}\left(A \rtimes_{\alpha, \mu} G\right) \rightarrow$ $\mathcal{F}_{\mu}(A)$ of Proposition 2.9 is surjective.

Proof. Let $\mathcal{U}$ be a neighborhood base of the identity $e \in G$ consisting of symmetric compact neighborhoods and let $\tilde{\varphi}_{U} \in C_{c}(G)^{+}$with $\operatorname{supp} \tilde{\varphi}_{U} \subseteq U, \tilde{\varphi}(t)=\tilde{\varphi}\left(t^{-1}\right)$ for all $t \in G$ and $\int_{G} \tilde{\varphi}_{U}(t) d t=1$. Let $\varphi_{U} \in C_{c}(G, A)$ be defined as $\varphi_{U}(t)=$ $\Delta(t)^{1 / 2} \alpha_{t^{-1}}(a) \tilde{\varphi}(t)$. It is then easy to check that $\left(\varphi_{U}\right)_{U \in \mathcal{U}}$ does the job.

Since, by Lemma 2.12, the inductive limit topology is stronger than the norm topology on $\mathcal{F}_{c}(A) \subseteq \mathcal{F}_{\mu}(A)$, it follows that $\Psi_{\mu}$ has dense image in $\mathcal{F}_{\mu}(A)$. Since it is isometric, it is surjective.

\section{Representations of GENERALIZED FIXED-POINT ALGEBRAS}

Assume that $A$ is a weakly proper $X \rtimes G$-algebra. In this section we describe the representations of generalized fixed-point algebras $A_{\mu}^{G, \alpha}$ - where $\|\cdot\|_{\mu}$ is some fixed crossed-product norm - via the induction process given by the partial $A_{\mu}^{G, \alpha}-$ $A \rtimes_{\alpha, \mu} G$ imprimitivity bimodule $\mathcal{F}_{\mu}(A)$. 
Recall that the (nondegenerate) representations of the maximal crossed product $A \rtimes_{\alpha} G$ are the integrated forms $\pi \rtimes U$ of covariant representations $(\pi, U)$ of the system $(A, G, \alpha)$. Throughout, we assume all representations to be nondegenerate and also allow representations on Hilbert modules (not only on Hilbert spaces).

Let $\|\cdot\|_{\mu}$ be a crossed-product norm on $C_{c}(G, A)$ and let $J_{\mu}$ be the kernel of the surjection $q_{\mu}: A \rtimes_{u} G \rightarrow A \rtimes_{\mu} G$. Then, representations of $A \rtimes_{\mu} G=$ $\left(A \rtimes_{u} G\right) / J_{\mu}$ correspond to representations of $A \rtimes_{u} G$ vanishing on $J_{\mu}$, i.e., covariant representations $(\pi, U)$ with $J_{\mu} \subseteq \operatorname{ker}(\pi \rtimes U)$. Such a representation $(\pi, U)$ will be called a $\mu$-covariant representation, and the corresponding representation of $A \rtimes_{\mu} G$ will be denoted by $\pi \rtimes_{\mu} U$.

Since $\mathcal{F}_{\mu}(A)$ is an imprimitivity $A_{\mu}^{G, \alpha}-\mathcal{I}_{A, \mu}$-bimodule, where $\mathcal{I}_{A, \mu}$ is the ideal $\left.\overline{\operatorname{span}}\{\langle\xi \mid \eta\rangle\rangle_{A \rtimes_{\mu} G}: \xi, \eta \in \mathcal{F}_{\mu}(A)\right\}$ of $A \rtimes_{\mu} G$, the representations of $A_{\mu}^{G, \alpha}$ are (up to unitary equivalence) the representations which are induced via $\mathcal{F}_{\mu}(A)$ from the nondegenerate representations of $\mathcal{I}_{A, \mu}$. Since every nondegenerate representation of an ideal uniquely extends to a representation of the full algebra, we may describe the representations of $A_{\mu}^{G, \alpha}$ as those representations which are induced from representations of $A \rtimes_{\mu} G$ which restrict to nondegenerate representations of $\mathcal{I}_{A, \mu}$.

For a given $\mu$-covariant representation $(\pi, U)$ of $(A, G, \alpha)$ on a Hilbert $B$-module $\mathcal{Y}$, recall that the representation of $A_{\mu}^{G, \alpha}$ induced from $\pi \rtimes_{\mu} U$ via $\mathcal{F}_{\mu}(A)$ acts on the Hilbert $B$-module $\mathcal{Y}_{\operatorname{Ind}(\pi \rtimes U)}:=\mathcal{F}_{\mu}(A) \otimes_{A \rtimes_{\mu} G} \mathcal{Y}$ via the action of $A_{\mu}^{G, \alpha}$ on $\mathcal{F}_{\mu}(A)$. The following result gives an explicit description of this representation.

Proposition 3.1. Let $(A, \alpha, \phi)$ be a weakly proper $X \rtimes G$-algebra, let $\|\cdot\|_{\mu}$ be a crossed-product norm on $C_{c}(G, A)$, and let $(\pi, U)$ be a $\mu$-covariant representation of $(A, G, \alpha)$ on a Hilbert $B$-module $\mathcal{Y}$. Let $\mathcal{H}_{0}:=\tilde{\pi}\left(C_{c}(X)\right) \mathcal{Y}$, where $\tilde{\pi}:=\pi \circ \phi$, equipped with the (semi-positive definite) B-valued inner product

$$
\langle\xi \mid \eta\rangle_{0}:=\int_{G} \Delta(t)^{-1 / 2}\left\langle\xi \mid U_{t} \eta\right\rangle_{B} d t
$$

and consider the left action of $A_{c}^{G, \alpha}$ on $\mathcal{H}_{0}$ given by

$$
m \cdot \xi=\pi(m) \xi
$$

where we extend $\pi$ to $\mathcal{M}(A)$ and use the fact that $A_{c}^{G, \alpha} \subseteq \mathcal{M}(A)$. Then this action extends to a representation $\operatorname{Ind}_{\mu}^{G, \alpha}(\pi \rtimes U)$ of $A_{\mu}^{G, \alpha}$ on the Hausdorff completion $\mathcal{H}=\operatorname{Ind}_{\mu}^{G, \alpha}(\mathcal{Y})$ of $\mathcal{H}_{0}$ which is unitarily equivalent to the induced representation $\operatorname{Ind}^{\mathcal{F}_{\mu}(A)}(\pi \rtimes U)$ of $A_{\mu}^{G, \alpha}$ on $\mathcal{Y}_{\operatorname{Ind}\left(\pi \rtimes_{\mu} U\right)}$ via $\mathcal{F}_{\mu}(A)$.

In particular, if $\pi \rtimes_{\mu} U$ is faithful (i.e., if $\operatorname{ker}(\pi \rtimes U)=J_{\mu}$ ), then $\operatorname{Ind}_{\mu}^{G, \alpha}$ is a faithful representation of $A_{\mu}^{G, \alpha}$.

Proof. First note that the integral in (3.2) exists: since $\xi, \eta \in \pi\left(C_{c}(X)\right) \mathcal{Y}$, we may choose $f, g \in C_{c}(X)$ such that $\xi=\tilde{\pi}(f) \xi$ and $\eta=\tilde{\pi}(g) \eta$. Then

$$
\begin{aligned}
\int_{G} \Delta(t)^{-1 / 2}\left\langle\xi \mid U_{t} \eta\right\rangle_{B} d t & =\int_{G} \Delta(t)^{-1 / 2}\left\langle\tilde{\pi}(f) \xi \mid U_{t} \tilde{\pi}(g) \eta\right\rangle_{B} d t \\
& =\int_{G} \Delta(t)^{-1 / 2}\left\langle\xi \mid \tilde{\pi}\left(\bar{f} \tau_{t}(g)\right) U_{t} \eta\right\rangle_{B} d t
\end{aligned}
$$

hence we integrate a continuous function with compact support. We should also remark that $m \cdot \xi \in \mathcal{H}_{0}$ for all $m \in A_{c}^{G, \alpha}$ and $\xi \in \mathcal{H}_{0}$. For this let $f \in C_{c}(X)$ such that $\xi=\tilde{\pi}(f) \xi$ and choose $g \in C_{c}(X)$ such that $g \cdot m \cdot f=m \cdot f$ (such $g$ exists since $\left.m \cdot f \in A_{c}\right)$. Then $m \cdot \xi=\tilde{\pi}(g) \pi(m \cdot f) \xi \in \mathcal{H}_{0}$. 
In a next step, we use the decomposition $\mathcal{F}_{\mu}(A)=\mathcal{F}(X) \otimes_{C_{0}(X) \rtimes G}\left(A \rtimes_{\mu} G\right)$ of Proposition 2.9 to see that

$$
\begin{aligned}
\mathcal{Y}_{\operatorname{Ind}\left(\pi \rtimes_{\mu} U\right)} & =\mathcal{F}_{\mu}(A) \otimes_{A \rtimes_{\mu} G} \mathcal{Y} \\
& \stackrel{\Phi}{\cong}\left(\mathcal{F}(X) \otimes_{C_{0}(X) \rtimes G}\left(A \rtimes_{\mu} G\right)\right) \otimes_{A \rtimes_{\mu} G} \mathcal{Y} \\
& =\mathcal{F}(X) \otimes_{C_{0}(X) \rtimes G}\left(\left(A \rtimes_{\mu} G\right) \otimes_{A \rtimes_{\mu} G} \mathcal{Y}\right) \\
& \stackrel{\Theta}{\cong} \mathcal{F}(X) \otimes_{C_{0}(X) \rtimes G} \mathcal{Y}
\end{aligned}
$$

Note that we denoted the first isomorphism in the above computation by $\Phi$ and the last one by $\Theta$. For bookkeeping, the inverse

$$
\Phi^{-1}: \mathcal{F}(X) \otimes_{C_{0}(X) \rtimes G}\left(A \rtimes_{\mu} G\right) \otimes_{A \rtimes_{\mu} G} \mathcal{Y} \rightarrow \mathcal{F}_{\mu}(A) \otimes_{A \rtimes_{\mu} G} \mathcal{Y}
$$

of the isomorphism $\Phi$ sends a triple elementary tensor

$$
f \otimes \varphi \otimes \xi \in C_{c}(X) \otimes C_{c}(G, A) \otimes \mathcal{Y}
$$

to the elementary tensor

$$
\Psi_{\mu}(f \otimes \varphi) \otimes \xi \in \mathcal{F}_{c}(A) \otimes \mathcal{Y}
$$

with $\Psi_{\mu}(f \otimes \varphi)=\int_{G} \Delta(t)^{-1 / 2} \alpha_{t}\left(f \cdot \varphi\left(t^{-1}\right)\right) d t$, and we have

$$
\Theta(f \otimes \varphi \otimes \xi)=f \otimes\left(\pi \rtimes_{\mu} U(\varphi) \xi\right) \in C_{c}(X) \otimes \mathcal{Y} .
$$

To proceed, we now consider the surjective linear map $V: \mathcal{F}_{c}(X) \odot \mathcal{Y} \rightarrow \mathcal{H}_{0}$ given on elementary tensors by $V(f \otimes \xi)=\tilde{\pi}(f) \xi$. A short computation yields

$$
\begin{aligned}
\langle f \otimes \xi \mid g \otimes \eta\rangle & =\langle\xi| \pi \rtimes_{\mu} U\left(\left\langle\langle f \mid g\rangle_{X}\right) \eta\right\rangle \\
& =\left\langle\xi \mid \int_{G} \Delta(t)^{-1 / 2} \tilde{\pi}\left(\bar{f} \tau_{t}(g)\right) U_{t} \eta d t\right\rangle \\
& =\int_{G} \Delta(t)^{-1 / 2}\left\langle\tilde{\pi}(f) \xi \mid U_{t} \tilde{\pi}(g) \eta\right\rangle d t \\
& =\langle\tilde{\pi}(f) \xi \mid \tilde{\pi}(g) \eta\rangle_{0}=\langle V(f \otimes \xi) \mid V(g \otimes \eta)\rangle_{0} .
\end{aligned}
$$

It follows that $\langle\cdot \mid \cdot\rangle_{0}$ is a positive semi-definite $B$-inner product on $\mathcal{H}_{0}$ such that $V$ extends to a Hilbert $B$-module isomorphism between $\mathcal{F}(X) \otimes_{C_{0}(X) \rtimes G} \mathcal{Y}$ and the Hausdorff completion of $\mathcal{H}_{0}$ with respect to this $B$-inner product. Thus, putting things together, we obtain an isomorphism of Hilbert $B$-modules

$$
V \circ \Theta \circ \Phi: \mathcal{Y}_{\operatorname{Ind}\left(\pi \rtimes_{\mu} U\right)} \rightarrow \mathcal{H}
$$

We now have to check that this isomorphism intertwines the induced representation Ind ${ }^{\mathcal{F}(A)}\left(\pi \rtimes_{\mu} U\right)$ with the representation determined by the left action of $A_{c}^{G, \alpha}$ on $\mathcal{H}_{0}$ as in (3.3). For this let $m \in A_{c}^{G, \alpha}$ and let $f \otimes \varphi \otimes \xi$ be a triple tensor in $C_{c}(X) \otimes C_{c}(G, A) \otimes \mathcal{Y} \subseteq \mathcal{F}(X) \otimes_{C_{0}(X) \rtimes G}\left(A \rtimes_{\mu} G\right) \otimes_{A \rtimes_{\mu} G} \mathcal{Y}$. We then get

$$
\operatorname{Ind}^{\mathcal{F}(A)}\left(\pi \rtimes_{\mu} U\right)(m)\left(\Phi^{-1}(f \otimes \varphi \otimes \xi)\right)=m \cdot \Psi_{\mu}(f \otimes \varphi) \otimes \xi .
$$

Choose a function $g \in C_{c}(X)$ such that $g \cdot m \cdot f=m \cdot f$, which exists since $m \cdot f \in A_{c}$. Using the $G$-invariance of $m$, we get

$$
\begin{aligned}
m \cdot \Psi_{\mu}(f \otimes \varphi) \otimes \xi & =\int_{G} \Delta(t)^{-1 / 2} \alpha_{t}\left(m \cdot f \cdot \varphi\left(t^{-1}\right)\right) d t \otimes \xi \\
& =\int_{G} \Delta(t)^{-1 / 2} \alpha_{t}\left(g \cdot m \cdot f \cdot \varphi\left(t^{-1}\right)\right) d t \otimes \xi \\
& =\Psi_{\mu}\left(g \otimes \iota_{A}^{\mu}(m \cdot f) \varphi\right) \otimes \xi \\
& =\Phi^{-1}\left(g \otimes \iota_{A}^{\mu}(m \cdot f) \varphi \otimes \xi\right) .
\end{aligned}
$$


where $\iota_{A}^{\mu}: A \rightarrow \mathcal{M}\left(A \rtimes_{\mu} G\right)$ denotes the canonical inclusion. The isomorphism $V \circ \Theta \circ \Phi: \mathcal{Y}_{\operatorname{Ind}\left(\pi \rtimes_{\mu} U\right)} \rightarrow \mathcal{H}$ sends this element to

$$
\begin{aligned}
V \circ \Theta\left(g \otimes \iota_{A}^{\mu}(m \cdot f) \varphi \otimes \xi\right) & =V\left(g \otimes \pi \rtimes_{\mu} U\left(\iota_{A}^{\mu}(m \cdot f) \varphi\right) \xi\right) \\
& =V\left(g \otimes \pi(m \cdot f) \pi \rtimes_{\mu} U(\varphi) \xi\right) \\
& =\pi(g \cdot m \cdot f) \pi \rtimes_{\mu} U(\varphi) \xi \\
& =\pi(m \cdot f) \pi \rtimes_{\mu} U(\varphi) \xi \\
& =\pi(m)\left(\tilde{\pi}(f) \pi \rtimes_{\mu} U(\varphi) \xi\right) \\
& =m \cdot(V \circ \Theta(f \otimes \varphi \otimes \xi)) .
\end{aligned}
$$

This completes the proof of the first assertion in the proposition. The final assertion follows from the fact that the induction process via imprimitivity bimodules preserves faithfulness.

Next, we are going to describe what happens if we induce to generalized fixedpoint algebras the covariant regular representation $\Lambda_{A}$ of $(A, \alpha)$ on the Hilbert $A$-module $L^{2}(G, A)$. Recall that $\Lambda_{A}$ is the integrated form of the covariant representation $(\tilde{\alpha}, 1 \otimes \lambda)$ on $L^{2}(G, A) \cong A \otimes L^{2} G$, where $\left.\tilde{\alpha}(a) \xi\right|_{t}:=\alpha_{t^{-1}}(a) \xi(t)$ for all $a \in A, t \in G$ and $\xi \in C_{c}(G, A) \subseteq L^{2}(G, A)$, and $\lambda: G \rightarrow \mathcal{U}\left(L^{2} G\right)$ is the (left) regular representation of $G$. Recall that $\operatorname{ker}\left(\Lambda_{A}\right)=J_{r} \supseteq J_{\mu}$ so that $(\tilde{\alpha}, 1 \otimes \lambda)$ is a $\mu$-representation for every crossed-product norm $\|\cdot\|_{\mu}$ on $C_{c}(G, A)$. Hence $\Lambda_{A}$ factors through a representation $\Lambda_{A}^{\mu}$ of $A \rtimes_{\mu} G$ on $L^{2}(G, A)$ for every $\mu$ and $\Lambda_{A}^{\mu}$ is faithful if and only if $\mu=r$. Of course, if $\Lambda_{A}$ is faithful, then $\Lambda_{A}^{\mu}$ is faithful for every $\mu$. The following result describes the induced representation $\operatorname{Ind}_{\mu}^{G, \alpha}\left(\Lambda_{A}\right)$ of $A_{\mu}^{G, \alpha}$.

Proposition 3.5. With notations as above, there is an isomorphism

$$
\Psi: \operatorname{Ind}_{\mu}^{G, \alpha}\left(L^{2}(G, A)\right) \stackrel{\sim}{\rightarrow} A
$$

of Hilbert $A$-modules which sends a function $\zeta \in C_{c}(X) \cdot C_{c}(G, A) \subseteq \operatorname{Ind}_{\mu}^{G, \alpha}\left(L^{2}(G, A)\right)$ to $\Psi(\zeta):=\int_{G} \Delta(t)^{-1 / 2} \zeta(t) d t \in A$.

Via this isomorphism, the representation $\operatorname{Ind}_{\mu}^{G, \alpha}\left(\Lambda_{A}\right)$ of $A_{\mu}^{G, \alpha}$ into $\mathcal{M}(A)=$ $\mathcal{L}_{A}(A)$ is given by the extension to $A_{\mu}^{G, \alpha}$ of the canonical inclusion map $A_{c}^{G, \alpha} \hookrightarrow$ $\mathcal{M}(A)$. In particular, $A_{r}^{G, \alpha}$ may be identified with the closure of $A_{c}^{G, \alpha}$ in $\mathcal{M}(A)$.

Proof. First notice that $\mathcal{H}_{c}:=C_{c}(X) \cdot C_{c}(G, A)$ is the (dense) subspace

$$
\left\{f \cdot \xi:=\tilde{\alpha}(\phi(f)) \cdot \xi: f \in C_{c}(X), \xi \in C_{c}(G, A)\right\}
$$

of $\mathcal{H}=\operatorname{Ind}_{\mu}^{G, \alpha}\left(L^{2}(G, A)\right)$. For $f \in C_{c}(X)$ and $\xi \in C_{c}(G, A)$, the function $\zeta=f \cdot \xi$ belongs to $C_{c}(G, A)$ and is given by $\zeta(t)=f \cdot \xi(t)=\alpha_{t^{-1}}(\phi(f)) \xi(t)$, so the integral $\psi(\zeta)=\int_{G} \Delta(t)^{-1 / 2} \zeta(t) d t$ makes sense and yields an element in $A$. To prove the first assertion of the proposition, it is enough to check that $\Psi$ preserves $A$-inner products and has dense range. Now, if $\eta, \zeta \in \mathcal{H}_{c}$, then

$$
\begin{aligned}
\langle\eta \mid \zeta\rangle_{0} & =\int_{G} \Delta(t)^{-1 / 2}\left\langle\eta \mid\left(1 \otimes \lambda_{t}\right) \zeta\right\rangle_{A} d t \\
& =\int_{G} \int_{G} \Delta(t)^{-1 / 2} \eta(s)^{*} \zeta\left(t^{-1} s\right) d t d s \\
& \stackrel{t}{ } \stackrel{s t^{-1}}{=} \int_{G} \int_{G} \Delta(s t)^{-1 / 2} \eta(s)^{*} \zeta(t) d t d s \\
& =\left(\int_{G} \Delta(s)^{-1 / 2} \eta(s) d t\right)^{*}\left(\int_{G} \Delta(t)^{-1 / 2} \zeta(t) d s\right) \\
& =\langle\Psi(\eta) \mid \Psi(\zeta)\rangle .
\end{aligned}
$$


Therefore, $\Psi$ preserves the $A$-valued inner products. To see that $\Psi$ has dense range, it is enough to approximate elements in $f \cdot a \in C_{c}(X) \cdot A$ by elements in $\operatorname{Im}(\Psi)$ in the norm of $A$. For this let $\epsilon>0$ and take a compact neighborhood $W$ of $e \in G$ with $\left\|\alpha_{t^{-1}}(\phi(f)) a-\phi(f) a\right\| \leq \epsilon$ for all $t \in W$. Now, take $h \in C_{c}(G)^{+}$with $\operatorname{supp}(h) \subseteq W$ and $\int_{G} \Delta(t)^{-1 / 2} h(t) d t=1$. Then, for $\xi(t):=h(t) a$, we have

$$
\begin{aligned}
\|\Psi(f \cdot \xi)-f \cdot a\| & =\left\|\int_{W} \Delta(t)^{-1 / 2}\left(\alpha_{t^{-1}}(\phi(f)) h(t) a-\phi(f) h(t) a\right) d t\right\| \\
& \leq \int_{W} \Delta(t)^{-1 / 2}\left\|\alpha_{t^{-1}}(\phi(f)) a-\phi(f) a\right\| h(t) d t \leq \epsilon
\end{aligned}
$$

This finishes the proof of the first assertion in the proposition. For the second, we have to check that $\Psi(m \cdot \zeta)=m \Psi(\zeta)$ for all $m \in A_{c}^{G, \alpha}$ and $\zeta \in \mathcal{H}_{c}$. But this follows from an easy computation using the fact that $m$ is $G$-invariant. The final assertion follows from Proposition 3.1 and the fact that $\Lambda_{A}$ factors through a faithful representation of $A \rtimes_{r} G$, so that $\operatorname{Ind}_{r}^{G, \alpha}$ is a faithful representation of $A_{r}^{G, \alpha}$. Since this representation extends the inclusion map $A_{c}^{G, \alpha} \hookrightarrow \mathcal{M}(A)$, the final assertion follows.

Remark 3.6. As a consequence of the above proposition we obtain the explicit description of the reduced generalized fixed-point algebra $A_{r}^{G, \alpha}$ as

$$
A_{r}^{G, \alpha}=C_{0}(G \backslash X) \cdot \overline{\left\{m \in \mathcal{M}(A)^{G, \alpha}: f \cdot m, m \cdot f \in A_{c} \forall f \in C_{c}(X)\right\}} \cdot C_{0}(G \backslash X),
$$

which, as it seems, has not been obtained so far in general. For centrally proper $X \rtimes G$-algebras (i.e., if $\left.\phi\left(C_{0}(X)\right) \subseteq Z \mathcal{M}(A)\right)$, this easily implies the description

$$
A_{r}^{G, \alpha}=C_{0}(G \backslash X) \cdot\left\{m \in \mathcal{M}(A)^{G, \alpha}: f \cdot m, m \cdot f \in A \forall f \in C_{0}(X)\right\},
$$

which is Kasparov's definition (see [20]) of the generalized fixed-point algebra in this situation. But in general one should be careful not to mistake the algebra in (3.7) with the algebra

$$
C_{0}(G \backslash X) \cdot\left\{m \in \mathcal{M}(A)^{G, \alpha}: f \cdot m, m \cdot f \in A \forall f \in C_{0}(X)\right\} \cdot C_{0}(G \backslash X),
$$

which, in general can be very different from $A_{r}^{G, \alpha}$. For example, if $G$ is a discrete group and $A=\mathcal{K}\left(L^{2} G\right)$ is equipped with the structure of a weakly proper $G \rtimes$ $G$-algebra with respect to the action $\alpha=\operatorname{Ad} \rho$ of $G$ on $A$, the right translation action of $G$ on $G$, and the representation $M: C_{0}(G) \rightarrow \mathcal{L}\left(L^{2} G\right)$ as multiplication operators, then (as we shall see in $\$ 4$ below) $A_{r}^{G, \alpha} \cong C_{r}^{*}(G)$, while the algebra in (3.9) equals the group von Neumann algebra $\mathcal{L}(G)$ of $G$. (Use $G \backslash G=\{$ pt $\}$ together with the fact that all multiplication operators $M(f)$ for $f \in C_{0}(G)$ are compact operators. It follows that the algebra in (3.9) is the commutator of the right regular representation of $G$.)

\section{LANDSTAD DUALITY FOR GENERAL COACTIONS}

As an application of the theory of generalized fixed-point algebras developed here, we now want to obtain a general version of Landstad duality for coactions. As a consequence, we shall prove some results on Katayama duality for coactions with respect to "intermediate" crossed products as studied in [15].

Our Landstad duality theorem extends Quigg's Landstad duality theorem for reduced (or normal) coactions (see 24]), and the work presented in this section is very much inspired by that result although the details of our proof differ substantially. Notice that a version of Landstad duality for maximal coactions is also known (see [18, Corollary 4.3]) and can be obtained from the version for normal coactions and the fact that taking maximalizations and normalizations yield equivalences between the categories of maximal and normal coactions ([18, Corollary A.3]). Our duality 
theorem will give an alternative direct proof which does not use this equivalence and also works for other exotic completions.

Recall that a coaction of a locally compact group $G$ on a $C^{*}$-algebra $B$ is a nondegenerate injective ${ }^{*}$-homomorphism $\delta: B \rightarrow \mathcal{M}\left(B \otimes C^{*}(G)\right)$ such that

$$
\left(\operatorname{id}_{B} \otimes \delta_{G}\right) \circ \delta=\left(\delta \otimes \operatorname{id}_{G}\right) \circ \delta
$$

as maps from $B$ to $\mathcal{M}\left(B \otimes C^{*}(G) \otimes C^{*}(G)\right)$, where $\delta_{G}: C^{*}(G) \rightarrow \mathcal{M}\left(C^{*}(G) \otimes\right.$ $\left.\mathcal{M}\left(C^{*}(G)\right)\right)$ is the integrated form of the strictly continuous homomorphism $G \ni$ $s \mapsto u_{s} \otimes u_{s} \in U \mathcal{M}\left(C^{*}(G) \otimes C^{*}(G)\right)$ and $s \mapsto u_{s}$ denotes the canonical inclusion of $G$ into the group $U \mathcal{M}\left(C^{*}(G)\right)$ of unitary multipliers of $C^{*}(G)$. Note that " $\otimes$ " denotes the minimal (or spatial) $C^{*}$-tensor product. We shall always assume that the coaction is nondegenerate in the sense that $\overline{\operatorname{span}}\left(\delta(B)\left(1 \otimes C^{*}(G)\right)\right)=B \otimes C^{*}(G)$.

In what follows we write $w_{G}: G \rightarrow U \mathcal{M}\left(C^{*}(G)\right)$ for the map $w_{G}(s)=u_{s}$. Then $w_{G}$ may be viewed as a unitary in $\mathcal{M}\left(C_{0}(G) \otimes C^{*}(G)\right) \cong C_{b}^{\mathrm{st}}\left(G, \mathcal{M}\left(C^{*}(G)\right)\right)$, the $C^{*}$-algebra of strictly continuous bounded functions $G \rightarrow \mathcal{M}\left(C^{*}(G)\right)$, and for any nondegenerate *-homomorphism $\sigma: C_{0}(G) \rightarrow \mathcal{M}(D)$, where $D$ is some $C^{*}$-algebra, we can consider the element $\sigma \otimes \operatorname{id}\left(w_{G}\right) \in U \mathcal{M}\left(D \otimes C^{*}(G)\right)$. Recall that a covariant homomorphism of the cosystem $(B, G, \delta)$ into $\mathcal{M}(D)$ consists of a pair $(\pi, \sigma)$ in which $\pi: B \rightarrow \mathcal{M}(D)$ and $\sigma: C_{0}(G) \rightarrow \mathcal{M}(D)$ are nondegenerate *-homomorphisms satisfying the covariance condition:

$$
\left(\pi \otimes \operatorname{id}_{G}\right) \circ \delta(b)=\left(\sigma \otimes \operatorname{id}_{G}\right)\left(w_{G}\right)(\pi(b) \otimes 1)\left(\sigma \otimes \operatorname{id}_{G}\right)\left(w_{G}\right)^{*} \quad \text { for all } b \in B .
$$

A crossed product of $(B, G, \delta)$ is a triple $\left(A, j_{B}, j_{C_{0}(G)}\right)$ consisting of a $C^{*}$-algebra $A$ together with a covariant homomorphism $\left(j_{B}, j_{C_{0}(G)}\right)$ of $(B, G, \delta)$ into $\mathcal{M}(A)$ such that

(1) $A=\overline{j_{B}(B) j_{C_{0}(G)}\left(C_{0}(G)\right)} \subseteq \mathcal{M}(A)$;

(2) For any covariant homomorphism $(\pi, \sigma)$ of $(B, G, \delta)$ into some $\mathcal{M}(D)$ there exists a *homomorphism $\pi \rtimes \sigma: A \rightarrow \mathcal{M}(D)$ such that $\pi=(\pi \rtimes \sigma) \circ j_{B}$ and $\sigma=(\pi \rtimes \sigma) \circ j_{C_{0}(G)}$.

A crossed product always exists and is unique up to isomorphism. We denote it by $\left(B \rtimes_{\delta} \widehat{G}, j_{B}, j_{C_{0}(G)}\right)$ (the notation $\widehat{G}$ indicates that a coaction crossed product should be regarded as a crossed product by a dual object of $G$-indeed, in case where $G$ is abelian, it is a crossed product by an action of the dual group $\widehat{G}$ ).

If $B \rtimes_{\delta} \widehat{G}$ is a coaction crossed product, there is a dual action $\widehat{\delta}: G \rightarrow \operatorname{Aut}\left(B \rtimes_{\delta} \widehat{G}\right)$ given by $\widehat{\delta}_{s}=j_{B} \rtimes\left(j_{C_{0}(G)} \circ \mathrm{r}_{s}\right)$ for $s \in G$, where $\mathrm{r}_{s} \in \operatorname{Aut}\left(C_{0}(G)\right)$ is the right translation action $\left(\mathrm{r}_{s}(f)\right)(t)=f(t s)$. With respect to this action, the canonical homomorphism $j_{C_{0}(G)}: C_{0}(G) \rightarrow \mathcal{M}\left(B \rtimes_{\delta} \widehat{G}\right)$ is $G$-equivariant, so that $\left(B \rtimes_{\delta} \widehat{G}, \widehat{\delta}, j_{C_{0}(G)}\right)$ is a weakly proper $G \rtimes G$-algebra. We always endow $B \rtimes_{\delta} \widehat{G}$ with this structure of a weakly proper $G \rtimes G$-algebra and we shall simply say weak $G \rtimes G$-algebra below.

By Katayama's duality theorem ([21), we always have a canonical surjective *-homomorphism

$$
\Phi_{B}: B \rtimes_{\delta} \widehat{G} \rtimes_{\widehat{\delta}} G \rightarrow B \otimes \mathcal{K}\left(L^{2} G\right)
$$

which is given as the integrated form of the covariant homomorphism $(\pi, U)$ of $\left(B \rtimes_{\delta} \widehat{G}, G, \widehat{\delta}\right)$ with

$$
\pi=\left(\operatorname{id}_{B} \otimes \lambda\right) \circ \delta \rtimes(1 \otimes M) \text { and } \quad U=1_{B} \otimes \rho
$$

where $\lambda$ and $\rho$ denote the left and right regular representations of $G$ and $M$ : $C_{0}(G) \rightarrow \mathcal{L}\left(L^{2} G\right)$ the representation by multiplication operators. For a quite detailed overview of the theory of co-systems and their crossed products we refer to [1]. We recall the following definition given in [10]: 
Definition 4.4. The coaction $\delta: B \rightarrow \mathcal{M}\left(B \otimes C^{*}(G)\right)$ is called maximal if the map $\Phi_{B}$ in (4.3) is an isomorphism. Moreover, the coaction $\delta$ is called normal if $\Phi_{B}$ factors through an isomorphism $\Phi_{B, r}: B \rtimes_{\delta} \widehat{G} \rtimes_{\widehat{\delta}, r} G \rightarrow B \otimes \mathcal{K}\left(L^{2} G\right)$.

Normal coactions have first been studied by Quigg in 25. He shows that a coaction $\delta$ is normal if and only if $j_{B}: B \rightarrow \mathcal{M}\left(B \rtimes_{\delta} \widehat{G}\right)$ is injective and that every coaction has a normalization which is given by the coaction $\delta_{r}: B_{r} \rightarrow$ $\mathcal{M}\left(B_{r} \otimes C^{*}(G)\right)$ on the quotient $B_{r}:=B / I$, where $I=\operatorname{ker} j_{B}$, given by the formula $\delta_{r}(b+I):=\left(q \otimes \operatorname{id}_{G}\right) \circ \delta(b)$, where $q: B \rightarrow B_{r}$ denotes the quotient map. This homomorphism induces an isomorphism $B \rtimes_{\delta} \widehat{G} \stackrel{\sim}{\longrightarrow} B_{r} \rtimes_{\delta_{r}} \widehat{G}$ of weak $G \rtimes G$-algebras. Moreover, if $\|\cdot\|_{\mu}$ is any crossed-product norm on $C_{c}(G, A)$ for a system $(A, G, \alpha)$ such that $A \rtimes_{\alpha, \mu} G$ admits a dual coaction $\widehat{\alpha}_{\mu}$, the dual coaction $\widehat{\alpha}_{r}$ on the reduced crossed product $A \rtimes_{\alpha, r} G$ is the normalization of $\widehat{\alpha}_{\mu}$.

On the other hand, it is shown in [10] that, for every coaction $\delta$, there exists a maximal coaction $\delta_{u}: B_{u} \rightarrow \mathcal{M}\left(B_{u} \otimes C^{*}(G)\right)$ such that $B=B_{u} / J$ for a suitable ideal $J$ in such a way that $\delta_{u}$ factors through $B$ to give the original coaction $\delta$ and such that the surjection $B_{u} \rightarrow B$ induces an isomorphism $B_{u} \rtimes_{\delta_{u}} \widehat{G} \cong B \rtimes_{\delta} \widehat{G}$ of weak $G \rtimes G$-algebras. The coaction $\left(B_{u}, \delta_{u}\right)$ is, up to isomorphism, uniquely determined by these properties and is called the maximalization of $(B, \delta)$. The construction of maximalizations given in [10] is quite involved and is not functorial. Our results below will give, in particular, an alternative functorial construction for maximalizations of arbitrary coactions. Note that the dual coaction $\widehat{\alpha}_{u}$ on the full crossed product $A \rtimes_{\alpha} G$ is the maximalization of a dual coaction $\widehat{\alpha}_{\mu}$ on an intermediate crossed product $A \rtimes_{\alpha, \mu} G$.

Definition 4.5. Let $\delta: B \rightarrow \mathcal{M}\left(B \otimes C^{*}(G)\right)$ be a coaction and let $\|\cdot\|_{\mu}$ be any crossed-product norm on $C_{c}\left(G, B \rtimes_{\delta} \widehat{G}\right)$, viewed as subalgebra of $\left(B \rtimes_{\delta} \widehat{G}\right) \rtimes_{\widehat{\delta}} G$. Then we say that $\delta$ satisfies $\mu$-duality or, shortly, that $\delta$ is a $\mu$-coaction, if the homomorphism $\Phi_{B}$ of (4.3) factors through an isomorphism

$$
\Phi_{B, \mu}: B \rtimes_{\delta} \widehat{G} \rtimes_{\widehat{\delta}, \mu} G \rightarrow B \otimes \mathcal{K}\left(L^{2} G\right) .
$$

A $\mu$-ization of $(B, \delta)$ is a $G$-coaction $\left(B_{\mu}, \delta_{\mu}\right)$ together with an isomorphism $B \rtimes_{\delta} \widehat{G} \cong$ $B_{\mu} \rtimes_{\delta_{\mu}} \widehat{G}$ of weak $G \rtimes G$-algebras in such a way that viewing $\|\cdot\|_{\mu}$ as a crossedproduct norm on $C_{c}\left(G, B_{\mu} \rtimes_{\delta_{\mu}} \widehat{G}\right)$ via this isomorphism, $\left(B_{\mu}, \delta_{\mu}\right)$ is a $\mu$-coaction.

Notice that in the above definition we do not require that $B \rtimes_{\delta} \widehat{G} \rtimes_{\widehat{\delta}, \mu} G$ carries a (bi)dual coaction - in fact the discussion below shows that this is automatic. For the reduced crossed-product norms, the above definition specializes to normal coactions and normalizations, i.e., an $r$-coaction is just a normal coaction and an $r$-ization is just a normalization. Similarly, for maximal crossed-product norms we get maximal coactions and maximalizations, i.e., a $u$-coaction is a maximal coaction and a $u$ ization is a maximalization of a given coaction.

It is clear that every coaction satisfies $\mu$-duality for some crossed-product norm $\|\cdot\|_{\mu}$, since the quotient $\left(B \rtimes_{\delta} \widehat{G} \rtimes_{\widehat{\delta}} G\right) / \operatorname{ker} \Phi_{B}$ always lies between the maximal and the reduced crossed product by $\widehat{\delta}$. On the other hand, if $\|\cdot\|_{\mu}$ is some crossed-product norm on $C_{c}\left(G, B \rtimes_{\delta} \widehat{G}\right)$ such that $(B, \delta)$ satisfies $\mu$-duality, then the canonical $G$-coaction on $B \otimes \mathcal{K}\left(L^{2} G\right)$ (see Equation (4.19)) may be viewed as a coaction for the double crossed product $B \rtimes_{\delta} \widehat{G} \rtimes_{\widehat{\delta}, \mu} G \cong B \otimes \mathcal{K}\left(L^{2} G\right)$. This coaction necessarily factors the bidual coaction on $B \rtimes_{\delta} \widehat{G} \rtimes_{\widehat{\delta}, u} G$. Conversely, we shall see below that for any given crossed product $B \rtimes_{\delta} \widehat{G} \rtimes_{\widehat{\delta}_{, \mu}} G$ admitting a (bi)dual coaction $\widehat{\widehat{\delta}}_{\mu}$, 
there exists a $\mu$-ization $\left(B_{\mu}, \delta_{\mu}\right)$ of $(B, \delta)$. This will lead, in particular, to a positive answer of Conjecture 6.14 in [15].

The main result in this section is the following theorem, which provides a general version of Landstad $\mu$-duality for coactions. Note that in case of normal coactions, Landstad duality has been obtained by Quigg in [24] (see also [17,18]).

In what follows, $C_{0}(G)$ will be always endowed with the right translation action r : $G \rightarrow \operatorname{Aut}\left(C_{0}(G)\right)$.

Theorem 4.6 (cf. [24, Theorem 3.3]). Suppose that $A$ is a weakly proper $G \rtimes$ $G$-algebra with respect to the $G$-equivariant structure map $\phi: C_{0}(G) \rightarrow \mathcal{M}(A)$ and the action $\alpha: G \rightarrow \operatorname{Aut}(A)$. Assume that $\|\cdot\|_{\mu}$ is a crossed-product norm on $C_{c}(G, A)$ which lies between $\|\cdot\|_{u}$ and $\|\cdot\|_{r}$ and which admits a dual coaction

$$
\widehat{\alpha}_{\mu}: A \rtimes_{\alpha, \mu} G \rightarrow \mathcal{M}\left(A \rtimes_{\alpha, \mu} G \otimes C^{*}(G)\right) .
$$

Let us write $B_{\mu}:=A_{\mu}^{G, \alpha}$ for any such $\|\cdot\|_{\mu}$. Then there is a canonical coaction $\delta_{\mathcal{F}_{\mu}(A)}$ of $G$ on $\mathcal{F}_{\mu}(A)$ (described in Lemma 4.12 below) which is compatible with the dual coaction $\widehat{\alpha}_{\mu}$ on $A \rtimes_{\alpha, \mu} G$ and therefore induces a compatible coaction $\delta_{\mu}$ on $B_{\mu} \cong \mathcal{K}\left(\mathcal{F}_{\mu}(A)\right)$ such that the following are true:

(1) The cosystem $\left(B_{\mu}, \delta_{\mu}\right)$ is Morita equivalent to $\left(A \rtimes_{\alpha, \mu} G, \widehat{\alpha}_{\mu}\right)$.

(2) $\left(B_{u}, \delta_{u}\right)$ is the maximalization of $\left(B_{\mu}, \delta_{\mu}\right)$ and $\left(B_{r}, \delta_{r}\right)$ is the normalization of $\left(B_{\mu}, \delta_{\mu}\right)$.

(3) The dual system $\left(B_{\mu} \rtimes_{\delta_{\mu}} \widehat{G}, G, \widehat{\delta}_{\mu}\right)$ is isomorphic to $(A, G, \alpha)$ as $G \rtimes G$-algebras via the covariant homomorphism $k \rtimes \phi: B_{\mu} \rtimes_{\delta_{\mu}} \widehat{G} \rightarrow A$, where $k: B_{\mu} \rightarrow$ $\mathcal{M}(A)$ extends the canonical inclusion $A_{c}^{G, \alpha} \hookrightarrow \mathcal{M}(A)$.

(4) $\left(B_{\mu}, G, \delta_{\mu}\right)$ satisfies $\mu$-duality and hence is a $\mu$-coaction.

Conversely, let $(B, \delta)$ be a $\mu$-coaction for some crossed-product norm $\|\cdot\|_{\mu}$ on $C_{c}\left(G, B \rtimes_{\delta} \widehat{G}\right)$ and let $B_{\mu}=A_{\mu}^{G, \alpha}$ for the weak $G \rtimes G$-algebra $(A, \alpha):=\left(B \rtimes_{\delta} \widehat{G}, \widehat{\delta}\right)$ equipped with the coaction $\delta_{\mu}$. Then $\left(B_{\mu}, \delta_{\mu}\right)$ is isomorphic to $(B, \delta)$.

Applying Theorem 4.6 to the weak $G \rtimes G$-algebra $\left(B \rtimes_{\delta} \widehat{G}, \widehat{\delta}\right)$ for a given coaction $\delta: B \rightarrow \mathcal{M}\left(B \otimes C^{*}(G)\right)$ gives:

Corollary 4.7. Suppose that $\|\cdot\|_{\mu}$ is any crossed-product norm on $C_{c}\left(G, B \rtimes_{\delta} \widehat{G}\right)$ such that the crossed product $B \rtimes_{\delta} \widehat{G} \rtimes_{\widehat{\delta}, \mu} G$ admits a dual coaction. Then $(B, \delta)$ admits a $\mu$-ization, i.e., there exists a coaction $\delta_{\mu}: B_{\mu} \rightarrow \mathcal{M}\left(B_{\mu} \otimes C^{*}(G)\right)$ "lying between" the maximalization $\delta_{u}$ and the normalization $\delta_{r}$ of $\delta$ such that $\delta_{\mu}$ satisfies $\mu$-duality and such that the dual systems $\left(B_{\mu} \rtimes_{\delta_{\mu}} \widehat{G}, G, \widehat{\delta}_{\mu}\right)$ and $\left(B \rtimes_{\delta} \widehat{G}, G, \widehat{\delta}\right)$ are isomorphic as weak $G \rtimes G$-algebras. If $\delta: B \rightarrow \mathcal{M}\left(B \otimes C^{*}(G)\right)$ is already a $\mu$-coaction, then $\left(B_{\mu}, \delta_{\mu}\right) \cong(B, \delta)$.

Recall that for a right Hilbert $B$-module $\mathcal{E}$, the multiplier module $\mathcal{M}(\mathcal{E})$ is defined as the set $\mathcal{L}_{B}(B, \mathcal{E})$ of adjointable operators from the standard Hilbert $B$-module $B$ into $\mathcal{E}$. The $B$-valued inner product on $\mathcal{E}$ then extends to an $\mathcal{M}(B)$-valued inner product on $\mathcal{M}(\mathcal{E})$ by the formula $\langle m \mid n\rangle_{\mathcal{M}(B)}=m^{*} \circ m \in \mathcal{L}_{B}(B)=\mathcal{M}(B)$. On the other hand, we get a left $\mathcal{M}\left(\mathcal{K}_{B}(\mathcal{E})\right)$-valued inner product by the formula $\mathcal{M}(\mathcal{K}(\mathcal{E}))\langle m \mid n\rangle=m \circ n^{*} \in \mathcal{L}_{B}(\mathcal{E})=\mathcal{M}\left(\mathcal{K}_{B}(\mathcal{E})\right)$. Note that there is a canonical inclusion map $\mathcal{E} \hookrightarrow \mathcal{M}(\mathcal{E})$ by identifying an element $\xi \in \mathcal{E}$ with the operator $b \mapsto$ $\xi \cdot b \in \mathcal{L}_{B}(B, \mathcal{E})$. The adjoint of this operator is given by $\eta \mapsto\langle\xi \mid \eta\rangle_{B} \in \mathcal{L}_{B}(\mathcal{E}, B)$. For more information on multiplier bimodules, see [1].

In what follows we write $\mathcal{E} \otimes D$ for the Hilbert $B \otimes D$-module which is obtained as the external (minimal) tensor product of the Hilbert $B$-module $\mathcal{E}$ with the $C^{*}$-algebra $D$, viewed as a Hilbert $D$-module.

To prove Theorem 4.6, we start with the following preliminary result: 
Lemma 4.8. Suppose that $A$ is a weakly proper $X \rtimes G$-algebra with respect to the action $\alpha: G \rightarrow \operatorname{Aut}(A)$ and the structure map $\phi: C_{0}(X) \rightarrow \mathcal{M}(A)$. Let $D$ be any $C^{*}$-algebra and consider $A \otimes D$ as a weakly proper $X \rtimes G$-algebra for the action $\alpha \otimes \mathrm{id}_{D}$ and the structure map $\phi \otimes 1$.

Then, for each given crossed-product norm $\|\cdot\|_{\mu}$ on $C_{c}(G, A)$, there exists a (unique) corresponding crossed-product norm $\|\cdot\|_{\nu}$ on $C_{c}(G, A \otimes D)$ such that the canonical inclusion $C_{c}(G, A) \odot D \subseteq C_{c}(G, A \otimes D)$ induces an isomorphism

$$
\left(A \rtimes_{\alpha, \mu} G\right) \otimes C^{*}(G) \cong(A \otimes D) \rtimes_{\alpha \otimes \mathrm{id}, \nu} G .
$$

The corresponding inclusion $\mathcal{F}_{c}(A) \otimes D \subseteq \mathcal{F}_{c}(A \otimes D)$ extends to an isomorphism

$$
\mathcal{F}_{\mu}(A) \otimes D \cong \mathcal{F}_{\nu}(A \otimes D)
$$

and, therefore, the inclusion of $A_{c}^{G, \alpha} \odot D$ into $(A \otimes D)_{c}^{G, \alpha \otimes \operatorname{id}_{D}}$ induces an isomorphism

$$
A_{\mu}^{G, \alpha} \otimes D \cong(A \otimes D)_{\nu}^{G, \alpha \otimes \mathrm{id}} .
$$

In particular, we may regard $\mathcal{F}_{c}(A \otimes D)$ as a dense submodule of $\mathcal{F}_{\mu}(A) \otimes D$ and $(A \otimes D)_{c}^{G, \alpha \otimes \operatorname{id}_{D}}$ as a dense subalgebra of $A_{\mu}^{G, \alpha} \otimes D$.

Remark 4.9. If $\|\cdot\|_{\mu}=\|\cdot\|_{r}$ is the reduced norm, then $\|\cdot\|_{\nu}=\|\cdot\|_{r}$ is also the reduced norm on $C_{c}(G, A \otimes D)$, which follows from the well-known isomorphism

$$
(A \otimes D) \rtimes_{\alpha \otimes \operatorname{id}_{D}, r} G \cong\left(A \rtimes_{\alpha, r} G\right) \otimes D .
$$

But we should point out that even if $\|\cdot\|_{\mu}=\|\cdot\|_{u}$ is the universal norm, we cannot expect in general that $\|\cdot\|_{\nu}$ is the universal norm as well. To see this consider the case where $A=\mathcal{K}\left(L^{2} G\right)$ with action $\alpha=\operatorname{Ad} \rho$ and structure map $M: C_{0}(G) \rightarrow \mathcal{L}\left(L^{2} G\right)$. Since $\alpha=\operatorname{Ad} \rho$ is implemented by a unitary representation, it is exterior equivalent to the trivial action of $G$ on $\mathcal{K}\left(L^{2} G\right)$. Thus we get

$$
\left(\mathcal{K}\left(L^{2} G\right) \otimes C^{*}(G)\right) \rtimes_{\operatorname{Ad} \rho \otimes \mathrm{id}, u} G \cong\left(\mathcal{K}\left(L^{2} G\right) \otimes C^{*}(G)\right) \otimes_{\max } C^{*}(G)
$$

while on the other side we have

$$
\left(\mathcal{K}\left(L^{2} G\right) \rtimes_{\operatorname{Ad} \rho, u} G\right) \otimes C^{*}(G) \cong\left(\mathcal{K}\left(L^{2} G\right) \otimes C^{*}(G)\right) \otimes C^{*}(G) .
$$

These algebras will be completions by different norms if the canonical quotient map $P: C^{*}(G) \otimes_{\max } C^{*}(G) \rightarrow C^{*}(G) \otimes C^{*}(G)$ is not an isomorphism, which is true for any group without Kirchberg's factorization property $(F)$ (see [22, §7]). Now, due to the work of Kirchberg and others, we know that there exist many groups which do not satisfy this property (see [1] for a survey on this property).

Proof of the lemma. Let $\left(\iota_{A}, \iota_{G}\right):(A, G) \rightarrow \mathcal{M}\left(A \rtimes_{\alpha} G\right)$ denote the canonical inclusions and let $j_{A \rtimes G}$ and $j_{D}$ denote the inclusions of $A \rtimes_{\alpha} G$ and $D$ into $\mathcal{M}\left(A \rtimes_{\alpha}\right.$ $G \otimes D)$, respectively. Then $\left(\left(j_{A \rtimes G} \circ \iota_{A}\right) \otimes j_{D}, \iota_{G} \otimes 1_{D}\right)$ is a covariant homomorphism of $(A \otimes D, G, \alpha \otimes$ id $)$ into $\mathcal{M}\left(A \rtimes_{\alpha} G \otimes D\right)$ whose integrated form $\Phi_{u}$ restricts to the inclusion $C_{c}(G, A) \odot D \hookrightarrow C_{c}(G, A \otimes D)$. Thus we see that

$$
\Phi_{u}:(A \otimes D) \rtimes_{\alpha \otimes \operatorname{id}_{D}, u} G \rightarrow\left(A \rtimes_{\alpha} G\right) \otimes D
$$

is a surjective *-homomorphism. Since $A \rtimes_{\alpha, \mu} G$ "lies" between $A \rtimes_{\alpha, u} G$ and $A \rtimes_{\alpha, r} G$, we conclude from this and (4.10) that $\left(A \rtimes_{\alpha, \mu} G\right) \otimes D$ "lies" between $(A \otimes D) \rtimes_{\alpha \otimes \operatorname{id}_{D}, u} G$ and $(A \otimes D) \rtimes_{\alpha \otimes \operatorname{id}_{D}, r} G$ and it follows that $\left(A \rtimes_{\alpha, \mu} G\right) \otimes D \cong$ $(A \otimes D) \rtimes_{\alpha \otimes \mathrm{id}, \nu} G$ for a suitable crossed-product norm $\|\cdot\|_{\nu}$.

Now, since $A \odot D$ is norm dense in $A \otimes D$, it follows that $\mathcal{F}_{c}(A) \odot D=C_{c}(X) A \odot D$ is inductive limit dense in $\mathcal{F}_{c}(A \otimes D)$, and since the $A \rtimes_{\alpha, \mu} G \otimes D$-valued inner product on $\mathcal{F}_{c}(A) \odot D$ coincides with the $(A \otimes D) \rtimes_{\alpha \otimes \operatorname{id}_{D}, \nu} G$-valued inner product by the choice of $\nu$, we see that $\mathcal{F}_{\mu}(A) \otimes D \cong \mathcal{F}_{\nu}(A \otimes D)$. The result follows.

We also need the following (certainly well-known) auxiliary result: 
Lemma 4.11. Suppose that $\mathcal{E}$ is a $B-C$ imprimitivity bimodule and that $\delta_{\mathcal{E}}$ is a coaction of $G$ on $\mathcal{E}$ which implements a Morita equivalence between coactions $\left(B, \delta_{B}\right)$ and $\left(C, \delta_{C}\right)$ of $G$. Then $\delta_{B}$ is maximal (resp. normal) if and only if $\delta_{C}$ is maximal (resp. normal) and the coaction $\left(\mathcal{E}, \delta_{\mathcal{E}}\right)$ factors through a Morita equivalence $\left(\mathcal{E}_{n}, \delta_{\mathcal{E}_{n}}\right)$ between the normalizations $\left(B_{n}, \delta_{B_{n}}\right)$ and $\left(C_{n}, \delta_{C_{n}}\right)$.

Proof. This follows from an easy linking algebra argument which we omit.

In the following lemma we allow a slightly more general situation than what we really need in this section, namely we assume that a closed subgroup $H$ of $G$ is given and consider a $G \rtimes H$-algebra $A$, i.e., a $C^{*}$-algebra $A$ endowed with an $H$-action $\alpha$ and an $H$-equivariant nondegenerate ${ }^{*}$-homomorphism $\phi: C_{0}(G) \rightarrow \mathcal{M}(A)$ (where $C_{0}(G)$ is now endowed with right translation $H$-action r). In addition, we assume that $\|\cdot\|_{\mu}$ is a $C^{*}$-norm on $C_{c}(H, A)$ (between $\|\cdot\|_{u}$ and $\|\cdot\|_{r}$ ) such that the dual coaction $\widehat{\alpha}: A \rtimes_{\alpha, u} H \rightarrow \mathcal{M}\left(A \rtimes_{\alpha, u} H \otimes C^{*}(H)\right)$ factors through a coaction

$$
\widehat{\alpha}_{\mu}: A \rtimes_{\alpha, \mu} H \rightarrow \mathcal{M}\left(A \rtimes_{\alpha, \mu} H \otimes C^{*}(H)\right) .
$$

We then consider the inflated $G$-coaction $\operatorname{Inf} \widehat{\alpha}_{\mu}:=\left(\operatorname{id} \otimes \iota_{G, H}\right) \circ \widehat{\alpha}_{\mu}: A \rtimes_{\alpha, \mu} H \rightarrow$ $\mathcal{M}\left(A \rtimes_{\alpha, \mu} H \otimes C^{*}(G)\right)$, where $\iota_{G, H}: C^{*}(H) \rightarrow \mathcal{M}\left(C^{*}(G)\right)$ denotes the canonical homomorphism defined as the integrated form of the obvious representation $H \rightarrow$ $U \mathcal{M}\left(C^{*}(G)\right)$ sending $t \in H$ to $u_{t} \in U \mathcal{M}\left(C^{*}(G)\right)$ (see [11 for more information on inflated coactions). This extra generality will be used in $\S 5$ of the forthcoming paper [9].

Lemma 4.12. Let $(A, \alpha)$ be a $G \rtimes H$-algebra as above, and let $\mathcal{F}_{\mu}(A)$ denote the corresponding Hilbert $A \rtimes_{\alpha, \mu} H$-module. Then there is a canonical Hilbert-module

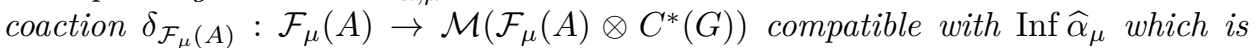
given, for $\xi \in \mathcal{F}_{c}(A)=\phi\left(C_{c}(G)\right) A$, by the formula

$$
\delta_{\mathcal{F}_{\mu}(A)}(\xi)=\phi \otimes \operatorname{id}_{G}\left(w_{G}\right)(\xi \otimes 1) .
$$

Moreover, via the isomorphism $\mathcal{F}_{\mu}(A) \cong \mathcal{F}(G) \otimes_{C_{0}(G) \rtimes H} A \rtimes_{\alpha, \mu} H$ of Proposition [2.9, the coaction $\delta_{\mathcal{F}_{\mu}(A)}$ corresponds to the (balanced) tensor product (as defined in [11, Proposition 2.13]) of the coactions $\delta_{\mathcal{F}(G)}$ and $\operatorname{Inf} \widehat{\alpha}$, where $\delta_{\mathcal{F}(G)}$ denotes the coaction of $G$ on $\mathcal{F}(G)=\mathcal{F}\left(C_{0}(G)\right)$ given by $\delta_{\mathcal{F}(G)}(f)=\omega_{G}(f \otimes 1)$ for all $f \in C_{c}(G)$.

Proof. We first need to check that the right hand side of the equation makes sense, i.e., that $\phi \otimes \operatorname{id}_{G}\left(w_{G}\right)(\xi \otimes 1)$ determines an adjointable operator from $A \rtimes_{\alpha, \mu} H \otimes$ $C^{*}(G)$ to $\mathcal{F}_{\mu}(A) \otimes C^{*}(G)$. For this we first observe that for any $z \in C^{*}(G)$ we get (with $\mu$ and $\nu$ as in Lemma 4.8 applied to $X=G$ and $H$ in place of $G$ ):

$$
\phi \otimes \operatorname{id}_{G}\left(w_{G}\right)(\xi \otimes z) \in \mathcal{F}_{c}\left(A \otimes C^{*}(G)\right) \subseteq \mathcal{F}_{\nu}\left(A \otimes C^{*}(G)\right) \cong \mathcal{F}_{\mu}(A) \otimes C^{*}(G) .
$$

Indeed, writing $\xi=\phi(f) a$ with $f \in C_{c}(X)$ and $a \in A$, we get

$$
\phi \otimes \operatorname{id}_{G}\left(w_{G}\right)(\xi \otimes z)=\phi \otimes \operatorname{id}_{G}\left(w_{G}(f \otimes z)\right)(a \otimes 1) \in \mathcal{F}_{c}\left(A \otimes C^{*}(G)\right),
$$

since $w_{G}(f \otimes z) \in C_{c}\left(G, C^{*}(G)\right)$. Suppose now that $w \in A \rtimes_{\alpha, \mu} H \otimes C^{*}(G)$. Factorizing $w=(1 \otimes z) w^{\prime}$ with $z \in C^{*}(G)$ and $w^{\prime} \in A \rtimes_{\alpha, \mu} H \otimes C^{*}(G)$, gives

$$
\delta_{\mathcal{F}_{\mu}(A)}(\xi) \cdot w=\phi \otimes \operatorname{id}_{G}\left(w_{G}\right)(\xi \otimes z) \cdot w^{\prime} \in \mathcal{F}_{\mu}(A) \otimes C^{*}(G) .
$$

It is straightforward to check that this does not depend on the given factorization $w=(1 \otimes z) w^{\prime}$ and that $w \mapsto \delta_{\mathcal{F}_{\mu}(A)}(\xi) \cdot w$ is adjointable with adjoint given by the formula

$$
\delta_{\mathcal{F}_{\mu}(A)}(\xi)^{*}(\eta)=\left\langle\left\langle\phi \otimes \operatorname{id}_{G}\left(\left(1 \otimes v^{*}\right) w_{G}\right)(\xi \otimes 1) \mid \eta^{\prime}\right\rangle\right\rangle_{A \rtimes_{\alpha, \mu} H \otimes C^{*}(G)}
$$

if we factorize $\eta \in \mathcal{F}_{\mu}(A) \otimes C^{*}(G)$ as $\eta=(1 \otimes v) \eta^{\prime}$ for some $v \in C^{*}(G)$. One may check, as above, that $\phi \otimes \operatorname{id}_{G}\left(\left(1 \otimes v^{*}\right) w_{G}\right)(\xi \otimes 1) \in \mathcal{F}_{c}\left(A \otimes C^{*}(G)\right)$, so that 
the inner product makes sense and gives an element in $\left(A \otimes C^{*}(G)\right) \rtimes_{\alpha \otimes \mathrm{id}, \nu} H \cong$ $\left(A \rtimes_{\alpha, \mu} H\right) \otimes C^{*}(G)$.

Note that it follows easily from (4.13) that $\delta_{\mathcal{F}_{\mu}(A)}\left(\mathcal{F}_{c}(A)\right)\left(1 \otimes C^{*}(G)\right)$ is dense in $\mathcal{F}_{c}\left(A \otimes C^{*}(G)\right)$ in the inductive limit topology and hence is dense in $\mathcal{F}_{\mu}(A) \otimes C^{*}(G)$. We now compute, for all $z, v \in C^{*}(G), \xi=\phi(f) a, \eta=\phi(g) b \in \mathcal{F}_{c}(A)$ and $t \in H$ :

$$
\begin{aligned}
& \left.\left(1 \otimes z^{*}\right)\left\langle\left\langle\delta_{\mathcal{F}_{\mu}(A)}(\xi) \mid \delta_{\mathcal{F}_{\mu}(A)}(\eta)\right\rangle\right\rangle_{\mathcal{M}(A \rtimes \mu} G \otimes C^{*}(G)\right) \\
& =\left\langle\left.\left\langle\delta_{\mathcal{F}_{\mu}(A)}(\xi)(1 \otimes z) \mid \delta_{\mathcal{F}_{\mu}(A)}(\eta)(1 \otimes v)\right\rangle_{A \rtimes_{\mu} G \otimes C^{*}(G)}\right|_{t}\right. \\
& =\Delta(t)^{-1 / 2}\left(\delta_{\mathcal{F}_{\mu}(A)}(\xi)(1 \otimes z)\right)^{*} \alpha_{t} \otimes \operatorname{id}_{G}\left(\delta_{\mathcal{F}_{\mu}(A)}(\eta)(1 \otimes v)\right) \\
& =\Delta(t)^{-1 / 2}\left(\phi \otimes \operatorname{id}_{G}\left(w_{G}\right)(\xi \otimes z)\right)^{*} \alpha_{t} \otimes \operatorname{id}_{G}\left(\phi \otimes \operatorname{id}_{G}\left(w_{G}\right)(\eta \otimes v)\right) \\
& =\Delta(t)^{-1 / 2}\left(a^{*} \otimes z^{*}\right)\left(\phi \otimes \operatorname{id}_{G}\left((\bar{f} \otimes 1) w_{G}^{*}\right)\right) \alpha_{t} \otimes \operatorname{id}_{G}\left(\phi \otimes \operatorname{id}_{G}\left(w_{G}(g \otimes 1)\right)(b \otimes v)\right) \\
& =\Delta(t)^{-1 / 2}\left(a^{*} \otimes z^{*}\right)\left(\phi \otimes \operatorname{id}_{G}\left(\left((\bar{f} \otimes 1) w_{G}^{*}\right) \mathrm{r}_{t} \otimes \operatorname{id}_{G}\left(w_{G}(g \otimes 1)\right)\right)\right)\left(\alpha_{t}(b) \otimes v\right)=\ldots
\end{aligned}
$$

Now observe that the middle part $\left((\bar{f} \otimes 1) w_{G}^{*}\right) \mathrm{r}_{t} \otimes \operatorname{id}_{G}\left(w_{G}(g \otimes 1)\right)$ is the function in $C_{c}^{\text {st }}\left(G, \mathcal{M}\left(C^{*}(G)\right)\right)$ given by

$$
s \mapsto \bar{f}(s) w_{G}^{*}(s) w_{G}(s t) g(s t)=\bar{f}(s) u_{s}^{*} u_{s t} g(s t)=\left(\bar{f} \mathrm{r}_{t}(g)\right)(s) u_{t} .
$$

Hence we can proceed the above computation with

$$
\begin{aligned}
& \ldots=\Delta(t)^{-1 / 2}\left(1 \otimes z^{*}\right)\left(\left(a^{*} \cdot \bar{f}\right) \alpha_{t}(g \cdot b) \otimes u_{t}\right)(1 \otimes v) \\
& =\left(1 \otimes z^{*}\right)\left(\langle\langle\xi \mid \eta\rangle\rangle_{A \rtimes_{\mu} G}(t) \otimes u_{t}\right)(1 \otimes v) \\
& =\left.\left(1 \otimes z^{*}\right) \operatorname{Inf} \widehat{\alpha}_{\mu}\left(\langle\langle\xi \mid \eta\rangle\rangle_{A \rtimes_{\mu} G}\right)(1 \otimes v)\right|_{t} .
\end{aligned}
$$

Since $z, v \in C^{*}(G)$ have been arbitrary, we see that

$$
\left\langle\left\langle\delta_{\mathcal{F}_{\mu}(A)}(\xi) \mid \delta_{\mathcal{F}_{\mu}(A)}(\eta)\right\rangle_{\mathcal{M}\left(A \rtimes_{\mu} G \otimes C^{*}(G)\right)}=\operatorname{Inf} \widehat{\alpha}_{\mu}(\langle\xi \mid \eta\rangle\rangle_{A \rtimes_{\mu} G}\right)
$$

for all $\xi, \eta \in \mathcal{F}_{c}(A)$. Since $\operatorname{Inf} \widehat{\alpha}_{\mu}$ is isometric, the same follows for $\delta_{\mathcal{F}_{\mu}(A)}$ with respect to the norm on $\mathcal{F}_{\mu}(A)$, so we see that $\delta_{\mathcal{F}_{\mu}(A)}$ extends uniquely to an isometric map

$$
\delta_{\mathcal{F}_{\mu}(A)}: \mathcal{F}_{\mu}(A) \rightarrow \mathcal{M}\left(\mathcal{F}_{\mu}(A) \otimes C^{*}(G)\right)
$$

such that $\overline{\operatorname{span}}\left(\delta_{\mathcal{F}_{\mu}(A)}\left(\mathcal{F}_{\mu}(A)\right)\left(1 \otimes C^{*}(G)\right)\right)=\mathcal{F}_{\mu}(A) \otimes C^{*}(G)$.

In order to complete the proof of the first part of the lemma, we only need to show that $\delta_{\mathcal{F}_{\mu}(A)}$ satisfies the coaction identity

$$
\left(\delta_{\mathcal{F}_{\mu}(A)} \otimes \operatorname{id}_{G}\right) \circ \delta_{\mathcal{F}_{\mu}(A)}=\left(\operatorname{id}_{\mathcal{F}_{\mu}(A)} \otimes \delta_{G}\right) \circ \delta_{\mathcal{F}_{\mu}(A)} .
$$

For this let $\xi=\phi(f) a \in \mathcal{F}_{c}(A)$ and $z \in C^{*}(G)$. As explained above, the element

$$
x:=\delta_{\mathcal{F}}(\xi)(1 \otimes z)=\phi \otimes \operatorname{id}\left(\omega_{G}\right)(\xi \otimes z) \in \mathcal{F}_{c}\left(A \otimes C^{*}(G)\right) \subseteq \mathcal{F}_{\nu}\left(A \otimes C^{*}(G)\right)
$$

is viewed as an element in $\mathcal{F}_{\mu}(A) \otimes C^{*}(G)$ via the canonical isomorphism $\mathcal{F}_{\nu}(A \otimes$ $\left.C^{*}(G)\right) \cong \mathcal{F}_{\mu}(A) \otimes C^{*}(G)$. For such elements we have

$$
\left(\delta_{\mathcal{F}_{\mu}(A)} \otimes \operatorname{id}_{G}\right)(x)=\left(\phi \otimes \operatorname{id}_{G}\left(\omega_{G}\right) \otimes 1\right)\left(\operatorname{id}_{\mathcal{F}} \otimes \sigma(x \otimes 1)\right),
$$

where $\sigma$ denotes the flip map on $C^{*}(G) \otimes C^{*}(G)$ and we use $\phi \otimes \operatorname{id}\left(\omega_{G}\right)(\xi \otimes z)$ in $\mathcal{F}_{c}\left(A \otimes C^{*}(G)\right) \subseteq \mathcal{M}\left(A \otimes C^{*}(G)\right)$. Indeed, this assertion follows from continuity of the involved maps and the fact that $x$ can be approximated, in the inductive limit topology, by elementary tensors of the form $\eta \otimes y \in \mathcal{F}_{c}(A) \otimes C^{*}(G)$. Moreover, 
using the relation $\left(\operatorname{id}_{C_{0}(G)} \otimes \delta_{G}\right)\left(\omega_{G}\right)=\left(\omega_{G} \otimes 1\right)\left(\operatorname{id}_{C_{0}(G)} \otimes \sigma\left(\omega_{G} \otimes 1\right)\right)$, we obtain

$$
\begin{aligned}
\left(\delta_{\mathcal{F}_{\mu}(A)} \otimes \operatorname{id}_{G}\right)\left(\delta_{\mathcal{F}_{\mu}(A)}(\xi)(1 \otimes z)\right) \\
=\left(\phi \otimes \operatorname{id}_{G}\left(w_{G}\right) \otimes 1\right)\left(\operatorname{id}_{A} \otimes \sigma\left(\phi \otimes \operatorname{id}_{G}\left(w_{G}\right)(\xi \otimes z \otimes 1)\right)\right) \\
=\left(\phi \otimes \operatorname{id}_{G}\left(w_{G}\right) \otimes 1\right)\left(\operatorname{id}_{A} \otimes \sigma\left(\phi \otimes \operatorname{id}_{G}\left(w_{G}\right)\right)\right)(\xi \otimes 1 \otimes z) \\
=\left(\phi \otimes \operatorname{id}_{G}\left(w_{G}\right) \otimes 1\right)\left(\phi \otimes \operatorname{id}_{G} \otimes \operatorname{id}_{G}\left(\operatorname{id}_{C_{0}(G)} \otimes \sigma\left(\omega_{G} \otimes 1\right)\right)\right)(\xi \otimes 1 \otimes z) \\
=\left(\phi \otimes \operatorname{id}_{G} \otimes \operatorname{id}_{G}\right)\left(\left(\omega_{G} \otimes 1\right)\left(\operatorname{id}_{C_{0}(G)} \otimes \sigma\left(\omega_{G} \otimes 1\right)\right)\right)(\xi \otimes 1 \otimes z) \\
=\left(\phi \otimes \operatorname{id}_{G} \otimes \operatorname{id}_{G}\right)\left(\operatorname{id}_{C_{0}(G)} \otimes \delta_{G}\left(\omega_{G}\right)\right)(\xi \otimes 1 \otimes z) \\
=\left(\operatorname{id}_{\mathcal{F}} \otimes \delta_{G}\right)\left(\phi \otimes \operatorname{id}_{G}\left(\omega_{G}\right)\right)(\xi \otimes 1 \otimes z) \\
=\left(\operatorname{id}_{\mathcal{F}} \otimes \delta_{G}\right)\left(\delta_{\mathcal{F}}(\xi)(1 \otimes z)\right) .
\end{aligned}
$$

Since $z \in C^{*}(G)$ was arbitrary, this gives the co-associativity of $\delta_{\mathcal{F}}$ and hence completes the proof of the first part of the lemma. For the final part, let us denote by $\Psi_{\mu}: \mathcal{F}(G) \otimes_{C_{0}(G) \rtimes H} A \rtimes_{\alpha, \mu} H \stackrel{\sim}{\rightarrow} \mathcal{F}_{\mu}(A)$ the isomorphism of Proposition 2.9 given by the formula $\Psi_{\mu}(f \otimes \varphi)=\int_{H} \Delta_{H}(t)^{-1 / 2} \alpha_{t}\left(f \cdot \varphi\left(t^{-1}\right)\right) d t$ for all $f \in C_{c}(G)$ and $\varphi \in C_{c}(H, A)$. The tensor product of the coactions $\delta_{\mathcal{F}(G)}$ and $\operatorname{Inf} \widehat{\alpha}_{\mu}$ will be denoted by $\tilde{\delta}$. It is given by $\tilde{\delta}(f \otimes \varphi)=\theta\left(\delta_{\mathcal{F}(G)}(f) \otimes \operatorname{Inf} \widehat{\alpha}_{\mu}(\varphi)\right)$, where

$$
\begin{aligned}
\theta:\left(\mathcal{F}(G) \otimes C^{*}(G)\right) \otimes_{C_{0}(G) \rtimes H \otimes C^{*}(G)} & \left(A \rtimes_{\alpha, \mu} H \otimes C^{*}(G)\right) \\
& \stackrel{\sim}{\longrightarrow}\left(\mathcal{F}(G) \otimes_{C_{0}(G) \rtimes H} A \rtimes_{\alpha, \mu} H\right) \otimes C^{*}(G)
\end{aligned}
$$

denotes the canonical isomorphism. We have

$$
\begin{aligned}
\left(\Psi_{\mu} \otimes \mathrm{id}\right)(\tilde{\delta} & (f \otimes \varphi)) \\
& =\int_{H} \Delta_{H}(t)^{-1 / 2}\left(\alpha_{t} \otimes \mathrm{id}\right)\left((\phi \otimes \mathrm{id})\left(\omega_{G}(f \otimes 1)\right)\left(\varphi\left(t^{-1}\right) \otimes u_{t^{-1}}\right)\right) d t \\
& =\int_{H} \Delta_{H}(t)^{-1 / 2}(\phi \otimes \mathrm{id})\left(\omega_{G}\right)\left(\phi\left(\tau_{t}(f)\right) \otimes u_{t}\right)\left(\alpha_{t}\left(\varphi\left(t^{-1}\right)\right) \otimes u_{t^{-1}}\right) d t \\
& =\int_{H} \Delta_{H}(t)^{-1 / 2}(\phi \otimes \mathrm{id})\left(\omega_{G}\right)\left(\alpha_{t}\left(f \cdot \varphi\left(t^{-1}\right)\right) \otimes 1\right) d t \\
& =\delta_{\mathcal{F}_{\mu}(A)}\left(\Psi_{\mu}(f \otimes \varphi)\right) .
\end{aligned}
$$

This shows that $\Psi_{\mu}$ is equivariant with respect to the coactions $\tilde{\delta}$ and $\delta_{\mathcal{F}_{\mu}(A)}$ and hence finishes the proof of the last statement in the lemma.

Remark 4.14. It is useful to obtain an explicit formula for the coaction $\delta_{\mu}: B_{\mu} \rightarrow$ $\mathcal{M}\left(B_{\mu} \otimes C^{*}(G)\right)$ on $B_{\mu}:=A_{\mu}^{H, \alpha}$ which is determined by the coaction $\delta_{\mathcal{F}_{\mu}(A)}$ of Lemma 4.12. We claim that it is given on the dense subalgebra $A_{c}^{H, \alpha}$ by the formula

$$
\delta_{\mu}(m)=\phi \otimes \operatorname{id}_{G}\left(w_{G}\right)(m \otimes 1) \phi \otimes \operatorname{id}_{G}\left(w_{G}^{*}\right)
$$

where we perform the formal computation inside $\mathcal{M}\left(A \otimes C^{*}(G)\right)$ but the outcome can be regarded as an element in $\mathcal{M}\left(B_{\mu} \otimes C^{*}(G)\right.$ ) since $\delta_{\mu}(m)$ (for $m \in A_{c}^{H, \alpha}$ )

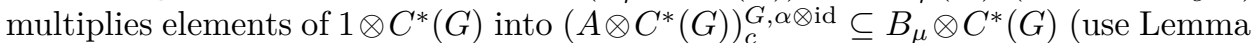
4.8). For a proof of (4.15), recall from Lemma 2.5 that $A_{c}^{H, \alpha}=\mathbb{E}\left(A_{c}\right)$ (where $\left.\mathbb{E}(a)=\int_{H}^{\mathrm{st}} \alpha_{t}(a) d t\right)$ with $A_{c}=C_{c}(G) \cdot A \cdot C_{c}(G)=\mathcal{F}_{c}(A) \cdot \mathcal{F}_{c}(A)^{*}$. Hence we get

$$
A_{c}^{H, \alpha}=\mathbb{E}\left(\mathcal{F}_{c}(A) \cdot \mathcal{F}_{c}(A)^{*}\right)={ }_{A_{c}^{H}}\left\langle\left\langle\mathcal{F}_{c}(A) \mid \mathcal{F}_{c}(A)\right\rangle\right\rangle .
$$


Thus we find $\xi, \eta \in \mathcal{F}_{c}(A)$ such that $m=\mathbb{E}\left(\xi \eta^{*}\right)={ }_{A_{\mu}^{G}}\langle\langle\xi \mid \eta\rangle\rangle$ and hence

$$
\begin{aligned}
\delta_{\mu}(m) & =\delta_{\mu}\left(A_{\mu}^{G}\langle\langle\xi \mid \eta\rangle\rangle\right)={ }_{\mathcal{M}\left(A_{\mu}^{G} \otimes C^{*}(G)\right)}\left\langle\left\langle\delta_{\mathcal{F}_{\mu}(A)}(\xi) \mid \delta_{\mathcal{F}_{\mu}(A)}(\eta)\right\rangle\right\rangle \\
& ={ }_{\mathcal{M}\left(A_{\mu}^{G} \otimes C^{*}(G)\right)}\left\langle\left\langle\phi \otimes \operatorname{id}_{G}\left(w_{G}\right)(\xi \otimes 1) \mid \phi \otimes \operatorname{id}_{G}\left(w_{G}\right)(\eta \otimes 1)\right\rangle\right\rangle \\
& =\int_{H}^{\mathrm{st}} \alpha_{t} \otimes \operatorname{id}_{G}\left(\phi \otimes \operatorname{id}_{G}\left(w_{G}\right)\left(\xi \eta^{*} \otimes 1\right) \phi \otimes \operatorname{id}_{G}\left(w_{G}^{*}\right)\right) d t .
\end{aligned}
$$

For fixed $t \in H$, we have $\alpha_{t} \otimes \operatorname{id}_{G}\left(\phi \otimes \operatorname{id}_{G}\left(w_{G}\right)\right)=\phi \otimes \operatorname{id}_{G}\left(\mathrm{r}_{t} \otimes \operatorname{id}_{G}\left(w_{G}\right)\right)$ and $\mathrm{r}_{t} \otimes \operatorname{id}_{G}\left(w_{G}\right)$ is the function $s \mapsto u_{s t}=u_{s} u_{t}$. Thus $\alpha_{t} \otimes \operatorname{id}_{G}\left(\phi \otimes \operatorname{id}_{G}\left(w_{G}\right)\right)=$ $\phi \otimes \operatorname{id}_{G}\left(w_{G}\right)\left(1 \otimes u_{t}\right)$. Using this identity, we get

$$
\begin{aligned}
\delta_{\mu}(m) & =\int_{H}^{\mathrm{st}}\left(\phi \otimes \operatorname{id}_{G}\left(w_{G}\right)\left(\alpha_{t}\left(\xi \eta^{*}\right) \otimes u_{t} u_{t}^{*}\right) \phi \otimes \operatorname{id}_{G}\left(w_{G}^{*}\right)\right) d t \\
& =\phi \otimes \operatorname{id}_{G}\left(w_{G}\right)\left(\int_{H}^{\mathrm{st}} \alpha_{t}\left(\xi \eta^{*}\right) d t \otimes 1\right) \phi \otimes \operatorname{id}_{G}\left(w_{G}^{*}\right) \\
& =\phi \otimes \operatorname{id}_{G}\left(w_{G}\right)(m \otimes 1) \phi \otimes \operatorname{id}_{G}\left(w_{G}^{*}\right)
\end{aligned}
$$

We should point out that a similar formula as in (4.15) is given for the reduced case in [18, Theorem 4.1].

We now return to the our original situation where $H=G$ and we use the above lemma in this case to prove the main result of this section:

Proof of Theorem 4.6. By Lemma 4.12 (applied to $H=G$ ) and the above remark we obtain a coaction $\delta_{\mu}$ on $B_{\mu}:=A_{\mu}^{G, \alpha}$ such that $\left(\mathcal{F}_{\mu}(A), \delta_{\mathcal{F}_{\mu}(A)}\right)$ implements a Morita equivalence between $\left(B_{\mu}, \delta_{\mu}\right)$ and $\left(A \rtimes_{\alpha, \mu} G, \widehat{\alpha}_{\mu}\right)$. Hence we get (1).

Statement (2) follows from Lemma 4.11 together with the fact that $\widehat{\alpha}_{u}$ is the maximalization of $\widehat{\alpha}_{\mu}$ and $\widehat{\alpha}_{r}$ is the normalization of $\widehat{\alpha}_{\mu}$.

In order to prove $(3)$ we first check that $(k, \phi)$ is a covariant homomorphism of $\left(B_{\mu}, G, \delta_{\mu}\right)$. In fact, for $m \in A_{c}^{G, \alpha}$ we have

$$
\left(k \otimes \operatorname{id}_{G}\right) \circ \delta_{\mu}(m)=\phi \otimes \operatorname{id}_{G}\left(w_{G}\right)(k(m) \otimes 1) \phi \otimes \operatorname{id}_{G}\left(w_{G}^{*}\right),
$$

which implies covariance of $(k, \phi)$. We also have

$$
k\left(B_{\mu}\right) \phi\left(C_{0}(G)\right) \supseteq A_{c}^{G, \alpha} \phi\left(C_{0}(G)\right),
$$

which is dense in $A$ by [24, Lemma 3.10 (2)], hence $A=k \rtimes \phi\left(B_{\mu} \rtimes_{\delta_{\mu}} \widehat{G}\right)$. Note that $k \rtimes \phi: B_{\mu} \rtimes_{\delta_{\mu}} G \rightarrow A$ is $G$-equivariant, since

$$
\begin{aligned}
k \rtimes \phi\left(\widehat{\delta}_{\mu}(s)\left(j_{B}(b) j_{C_{0}(G)}(f)\right)\right) & \left.=k \rtimes \phi\left(j_{B}(b) j_{C_{0}(G)}\left(\mathrm{r}_{s}(f)\right)\right)\right) \\
& =k(b) \phi\left(\mathrm{r}_{s}(f)\right)=\alpha_{s}(k(b) \phi(f)) \\
& =\alpha_{s}\left(k \rtimes \phi\left(j_{B}(b) j_{C_{0}(G)}(f)\right)\right) .
\end{aligned}
$$

To see that $k \rtimes \phi: B_{\mu} \rtimes_{\delta_{\mu}} \widehat{G} \rightarrow A$ is an isomorphism we use the fact that the crossed product by a coaction is always isomorphic to the crossed product by its normalization. Moreover, $(k, \phi)$ factors through the covariant homomorphism (id, $\phi)$ of $\left(B_{r}, G, \delta_{r}\right)$ and it follows then from the $G$-equivariance checked above and [24, Proposition 3.1] that id $\rtimes \phi: B_{r} \rtimes_{\delta_{r}} \widehat{G} \rightarrow A$ is an isomorphism.

We finally have to show that $\left(B_{\mu}, \delta_{\mu}\right)$ satisfies $\mu$-duality. For this we have to show that the canonical map $\Phi_{B_{\mu}}: B_{\mu} \rtimes_{\delta_{\mu}} \widehat{G} \rtimes_{\widehat{\delta}_{\mu}, u} G \rightarrow B_{\mu} \otimes \mathcal{K}\left(L^{2} G\right)$ factors through an isomorphism $B_{\mu} \rtimes_{\delta_{\mu}} \widehat{G} \rtimes_{\widehat{\delta}_{\mu}, \mu} G \cong B_{\mu} \otimes \mathcal{K}\left(L^{2} G\right)$. Since $\delta_{u}$ is the maximalization of $\delta_{\mu}$, we have an isomorphism

$$
\Phi_{B_{u}}: B_{\mu} \rtimes_{\delta_{\mu}} \widehat{G} \rtimes_{\widehat{\delta}_{\mu}, u} G \cong A \rtimes_{\alpha} G \stackrel{\sim}{\longrightarrow} B_{u} \otimes \mathcal{K}\left(L^{2} G\right) .
$$


Combining the Morita equivalence $B_{u} \sim_{M} A \rtimes_{\alpha} G$ with this isomorphism, we obtain a Morita equivalence $B_{u} \sim_{M} B_{u} \otimes \mathcal{K}\left(L^{2} G\right)$ given by the equivalence bimodule $\mathcal{F}_{u}(A) \otimes_{A \rtimes_{\alpha} G}\left(B_{u} \otimes \mathcal{K}\left(L^{2} G\right)\right)$. To finish, we need:

Lemma 4.16. The $B_{u}-B_{u} \otimes \mathcal{K}\left(L^{2} G\right)$-equivalence bimodule $\mathcal{F}_{u}(A) \otimes_{A \rtimes_{\alpha} G}\left(B_{u} \otimes\right.$ $\left.\mathcal{K}\left(L^{2} G\right)\right)$ is isomorphic to $B_{u} \otimes \mathcal{F}(G)$, where we regard $\mathcal{F}(G)$ as a $\mathbb{C}-\mathcal{K}\left(L^{2} G\right)$ equivalence bimodule with respect to the isomorphism $M \rtimes \rho: C_{0}(G) \rtimes G \rightarrow \mathcal{K}\left(L^{2} G\right)$.

Proof. By Proposition 2.9, we have

$$
\mathcal{F}_{u}(A) \cong \mathcal{F}(G) \otimes_{C_{0}(G) \rtimes G}\left(A \rtimes_{\alpha} G\right) \cong \mathcal{F}(G) \otimes_{C_{0}(G) \rtimes G}\left(B_{u} \otimes \mathcal{K}\left(L^{2} G\right)\right),
$$

where in the last isomorphism we replaced $A \rtimes_{\alpha} G=\left(B_{u} \rtimes_{\delta_{u}} \widehat{G}\right) \rtimes_{\widehat{\delta}_{u}, u} G$ by the isomorphic algebra $B_{u} \otimes \mathcal{K}\left(L^{2} G\right)$ via $\Phi_{B_{u}}$. We need to understand the left action of $C_{0}(G) \rtimes G$ on $B_{u} \otimes \mathcal{K}\left(L^{2} G\right)$. The left action of $C_{0}(G) \rtimes G$ on $A \rtimes_{\alpha} G$ is given by the integrated form of the covariant homomorphism $\left(\iota_{A} \circ \phi, \iota_{G}\right)$, where $\left(\iota_{A}, \iota_{G}\right)$ : $(A, G) \rightarrow \mathcal{M}\left(A \rtimes_{\alpha} G\right)$ denote the canonical maps. Identifying $B_{u} \rtimes_{\delta_{u}} \widehat{G}$ with $A$ via $k \rtimes \phi$ as in Theorem 4.6, the corresponding action of $C_{0}(G) \rtimes G$ on $\left(B_{u} \rtimes_{\delta_{u}} \widehat{G}\right) \rtimes_{\widehat{\delta}_{u}, u} G$ is given by the covariant homomorphism $\left(i_{B_{u} \rtimes_{\delta_{u}} \widehat{G}^{\circ}}^{\circ} j_{C_{0}(G)}, \iota_{G}\right)$. If we compose this with the isomorphism $\Phi_{B_{u}}=\left(\left(\operatorname{id}_{B_{u}} \otimes \lambda\right) \circ \delta_{u} \rtimes\left(1_{B_{u}} \otimes M\right)\right) \rtimes\left(1_{B_{u}} \otimes \rho\right)$, we see that the action of $C_{0}(G) \rtimes G$ on $B_{u} \otimes \mathcal{K}\left(L^{2} G\right)$ is given by $1_{B_{u}} \otimes(M \rtimes \rho)$. Since $M \rtimes \rho: C_{0}(G) \rtimes G \rightarrow \mathcal{K}\left(L^{2} G\right)$ is an isomorphism, we get

$$
\mathcal{F}_{u}(A) \cong \mathcal{F}(G) \otimes_{C_{0}(G) \rtimes G}\left(B_{u} \otimes \mathcal{K}\left(L^{2} G\right)\right) \cong B_{u} \otimes \mathcal{F}(G)
$$

if we understand $\mathcal{F}(G)$ as a Hilbert $\mathbb{C}-\mathcal{K}\left(L^{2} G\right)$-bimodule via the isomorphism $M \rtimes \rho: C_{0}(G) \rtimes G \rightarrow \mathcal{K}\left(L^{2} G\right)$.

We can now finish the proof of Theorem 4.6 as follows: it is clear that $B_{\mu}$ is the quotient of $B_{u}$ corresponding to the quotient $B_{\mu} \otimes \mathcal{K}\left(L^{2} G\right)$ of $B_{u} \otimes \mathcal{K}\left(L^{2} G\right)$ under the Rieffel-correspondence for the equivalence bimodule $B_{u} \otimes \mathcal{F}(G)$. By the lemma, this module is isomorphic to $\mathcal{F}_{u}(A)$ if we identify $A \rtimes_{\alpha} G$ with $B_{u} \otimes \mathcal{K}\left(L^{2} G\right)$ via $\Phi_{B_{u}}$. But the quotient of $A \rtimes_{\alpha} G$ corresponding to $B_{\mu}$ with respect to $\mathcal{F}_{u}(A)$ is $A \rtimes_{\alpha, \mu} G=\left(B_{\mu} \rtimes_{\delta_{\mu}} G\right) \rtimes_{\widehat{\delta}_{\mu}, \mu} G$ which implies that $\Phi_{B_{u}}$ factors through an isomorphism $\Phi_{B_{\mu}}$ between $\left(B_{\mu} \rtimes_{\delta_{\mu}} G\right) \rtimes_{\widehat{\delta}_{\mu}, \mu} G$ and $B_{\mu} \otimes \mathcal{K}\left(L^{2} G\right)$.

As a consequence of our previous results, we see that for a weak $G \rtimes G$-algebra $A$, the Morita equivalence $A_{\mu}^{G, \alpha} \sim_{M} A \rtimes_{\alpha, \mu} G$ implemented by $\mathcal{F}_{\mu}(A)$ is actually a canonical stable isomorphism:

Corollary 4.17. Let $(A, \alpha)$ be a weak $G \rtimes G$-algebra and let $\|\cdot\|_{\mu}$ be a crossedproduct norm on $C_{c}(G, A)$ for which the dual coaction on $A \rtimes_{\alpha, u} G$ factors through a dual coaction on $A \rtimes_{\alpha, \mu} G$. Then $A \rtimes_{\alpha, \mu} G \cong A_{\mu}^{G, \alpha} \otimes \mathcal{K}\left(L^{2} G\right)$.

Proof. By Theorem 4.6, we have $(A, \alpha) \cong\left(A_{\mu}^{G, \alpha} \rtimes_{\delta_{\mu}} \widehat{G}, \widehat{\delta}_{\mu}\right)$ as $G \rtimes G$-algebras and $\left(A_{\mu}^{G, \alpha}, \delta_{\mu}\right)$ satisfies $\mu$-duality, so that

$$
A \rtimes_{\alpha, \mu} G \cong A_{\mu}^{G, \alpha} \rtimes_{\delta_{\mu}} \widehat{G} \rtimes_{\widehat{\delta}_{\mu}, \mu} G \cong A_{\mu}^{G, \alpha} \otimes \mathcal{K}\left(L^{2} G\right) .
$$

The proof of the final converse statement in Theorem 4.6 will now be a consequence of the following variant of Lemma 4.16. It also shows that the isomorphism $A \rtimes_{\alpha, \mu} G \cong A_{\mu}^{G, \alpha} \otimes \mathcal{K}\left(L^{2} G\right)$ of the above lemma turns the Morita equivalence $A_{\mu}^{G} \sim_{M} A \rtimes_{\alpha, \mu} G$ into the canonical one: 
Lemma 4.18. Suppose that $\delta: B \rightarrow M\left(B \otimes C^{*}(G)\right)$ is a $\mu$-coaction for some given crossed-product norm $\|\cdot\|_{\mu}$ on $C_{c}\left(G, B \rtimes_{\delta} \widehat{G}\right)$. Recall that this means that the canonical homomorphism

$$
\Phi: B \rtimes_{\delta} \widehat{G} \rtimes_{\hat{\delta}} G \rightarrow B \otimes \mathcal{K}\left(L^{2} G\right)
$$

factors through an isomorphism $\Phi_{\mu}: B \rtimes_{\delta} \widehat{G} \rtimes_{\hat{\delta}, \mu} G \stackrel{\sim}{\rightarrow} B \otimes \mathcal{K}\left(L^{2} G\right)$. Let the crossed product $\left(B \rtimes_{\delta} \widehat{G}, \widehat{\delta}\right)$ be equipped with the canonical weak $G \rtimes G$-algebra structure. Then there is a canonical isomorphism

$$
\mathcal{F}_{\mu}\left(B \rtimes_{\delta} \widehat{G}\right) \cong B \otimes \mathcal{F}(G)
$$

as Hilbert $B \otimes \mathcal{K}\left(L^{2} G\right)$-modules if we identify $C_{0}(G) \rtimes G$ with $\mathcal{K}\left(L^{2} G\right)$ via $M \rtimes \rho$ and $B \rtimes_{\delta} \widehat{G} \rtimes_{\widehat{\delta}, \mu} G$ with $B \otimes \mathcal{K}\left(L^{2} G\right)$ via $\Phi_{\mu}$. In particular, the left action of $\left(B \rtimes_{\delta} \widehat{G}\right)_{c}^{G, \widehat{\delta}}$ on this module extends to an isomorphism

$$
\left(B \rtimes_{\delta} \widehat{G}\right)_{\mu}^{G, \widehat{\delta}}=\mathcal{K}\left(\mathcal{F}_{\mu}\left(B \rtimes_{\delta} \widehat{G}\right)\right) \cong B .
$$

This isomorphism sends the coaction $\delta_{\mu}^{G}$ on $\left(B \rtimes_{\delta} \widehat{G}\right)_{\mu}^{G, \widehat{\delta}}$ (given by Theorem 4.6) to the original coaction $\delta$ on $B$.

Proof. The proof of the first assertion is word for word as the proof of Lemma 4.16 in case where $A=B \rtimes_{\delta} \widehat{G}$ and where we replace $B_{u}$ by $B$. This gives the chain of isomorphisms

$$
\begin{aligned}
\mathcal{F}_{\mu}\left(B \rtimes_{\delta} \widehat{G}\right) & \cong \mathcal{F}_{\mu}\left(B \rtimes_{\delta} \widehat{G}\right) \otimes_{B \rtimes_{\delta} \widehat{G} \rtimes_{\hat{\delta}, \mu} G}\left(B \otimes \mathcal{K}\left(L^{2} G\right)\right) \\
& \cong \mathcal{F}(G) \otimes_{C_{0}(G) \rtimes G}\left(B \otimes \mathcal{K}\left(L^{2} G\right)\right) \cong B \otimes \mathcal{F}(G) .
\end{aligned}
$$

Now, it is well-known (see Equation (2.3) in [10]) that the canonical isomorphism $B \rtimes_{\delta} \widehat{G} \rtimes_{\hat{\delta}, \mu} G \cong B \otimes \mathcal{K}\left(L^{2} G\right)$ sends the bidual coaction to the coaction on $B \otimes \mathcal{K}\left(L^{2} G\right)$ given by the formula

$$
\delta_{B \otimes \mathcal{K}}(x)=\left(1 \otimes \omega_{G}^{*}\right)\left(\delta \otimes_{*} \text { id }\right)(x)\left(1 \otimes \omega_{G}\right) \quad \text { for all } x \in B \otimes \mathcal{K}\left(L^{2} G\right),
$$

where $\delta \otimes_{*}$ id $:=\sigma \circ(\delta \otimes$ id $): B \otimes \mathcal{K}\left(L^{2} G\right) \rightarrow \mathcal{M}\left(B \otimes \mathcal{K}\left(L^{2} G\right) \otimes C^{*}(G)\right)$ and $\sigma: C^{*}(G) \otimes$ $\mathcal{K}\left(L^{2} G\right) \rightarrow \mathcal{K}\left(L^{2} G\right) \otimes C^{*}(G)$ is the flip map. By Lemma 4.12, the coaction $\delta_{\mathcal{F}_{\mu}(A)}$ on $\mathcal{F}_{\mu}\left(B \rtimes_{\delta} \widehat{G}\right)$ corresponds to the coaction on $\mathcal{F}(G) \otimes_{C_{0}(G) \rtimes G}\left(B \otimes \mathcal{K}\left(L^{2} G\right)\right)$ which is the (balanced) tensor product (as in [11, Proposition 2.13]) of the coactions $\delta_{\mathcal{F}(G)}(f)=\omega_{G}(f \otimes 1)$ on $\mathcal{F}(G)$ and the coaction $\delta_{B \otimes \mathcal{K}}$ on $B \otimes \mathcal{K}\left(L^{2} G\right)$. And it is easy to see that the canonical isomorphism $\mathcal{F}(G) \otimes_{C_{0}(G) \rtimes G}\left(B \otimes \mathcal{K}\left(L^{2} G\right)\right) \cong B \otimes \mathcal{F}(G)$ sends this tensor product coaction to the coaction on $B \otimes \mathcal{F}(G) \cong B \otimes \mathcal{F}(G)$ given by the formula

$$
\delta_{B \otimes \mathcal{F}(G)}(b \otimes f):=\left(\delta \otimes_{*} \text { id }\right)(b \otimes f)\left(1 \otimes \omega_{G}\right) \quad \text { for all } f \in C_{c}(G), b \in B .
$$

Finally, a simple computation shows that the induced coaction on $B \cong \mathcal{K}(B \otimes \mathcal{F}(G))$ coincides with the original coaction $\delta$ on $B$. This proves the last statement of the lemma since the coaction $\delta_{\mu}^{G}$ on $\left(B \rtimes_{\delta} \widehat{G}\right)_{\mu}^{G, \widehat{\delta}}$ is, by definition, the coaction induced by $\delta_{\mathcal{F}_{\mu}(A)}$ on $\mathcal{K}\left(\mathcal{F}_{\mu}(A)\right) \cong \mathcal{K}(B \otimes \mathcal{F}(G))$.

\section{E-DUAlity fOR IDEALS IN $B(G)$}

In the previous section we considered arbitrary crossed-product norms $\|\cdot\|_{\mu}$ on $C_{c}(G, A)$ such that the corresponding crossed product $A \rtimes_{\alpha, \mu} G$ admits a dual coaction. In [15] it is shown that if $E$ is a $G$-invariant weak*-closed ideal in the Fourier-Stieltjes algebra $B(G)$, then $E$ determines a crossed-product norm $\|\cdot\|_{E}$ on $C_{c}(G, A)$ for any action $\alpha: G \rightarrow \operatorname{Aut}(A)$ which admits a dual coaction $\widehat{\alpha}_{E}$. 
This allows us to consider functorial properties of the $E$-crossed product functor $(A, G, \alpha) \mapsto A \rtimes_{\alpha, E} G$. Indeed, it is possible to show that the construction $(A, G, \alpha) \mapsto A \rtimes_{\alpha, E} G$ is a functor between suitable categories and Proposition 5.2 below already indicates some steps in this direction.

Recall that $B(G)$ consists of all functions of the form $s \mapsto\langle\pi(s) \xi \mid \eta\rangle$ in which $\pi$ : $G \rightarrow \mathcal{U}\left(H_{\pi}\right)$ is a unitary representation of $G$ and $\xi, \eta \in H_{\pi}$. It can be identified with the space $C^{*}(G)^{*}$ of continuous linear functionals on $C^{*}(G)$ via $f(x)=\langle\pi(x) \xi \mid \eta\rangle$ if $f(s)=\langle\pi(s) \xi \mid \eta\rangle$ for all $s \in G$. The weak*-topology on $B(G)$ is the one coming from this identification. For any non-zero $G$-invariant weak*-closed ideal $E \subseteq B(G)$ let $I_{E}={ }^{\perp} E:=\left\{a \in C^{*}(G): f(a)=0\right.$ for all $\left.f \in E\right\}$. It is shown in 15, Lemma 3.1 and Lemma 3.14] that $I_{E}$ is a closed ideal in $C^{*}(G)$ which is contained in the kernel ker $\lambda$ of the regular representation of $G$, and Kaliszewski, Quigg and Landstad define the E-group $C^{*}$-algebra of $G$ as the quotient $C^{*}$-algebra

$$
C_{E}^{*}(G):=C^{*}(G) / I_{E}
$$

(see [15, Definition 3.2]). Let $q_{E}: C^{*}(G) \rightarrow C_{E}^{*}(G)$ denote the quotient map. If $\alpha: G \rightarrow \operatorname{Aut}(A)$ is an action, then Kaliszewski, Quigg and Landstad define the E-crossed product $A \rtimes_{\alpha, E} G$ as

$$
A \rtimes_{\alpha, E} G=\left(A \rtimes_{\alpha} G\right) / J_{\alpha, E} \quad \text { with } \quad J_{\alpha, E}=\operatorname{ker}\left(\mathrm{id} \otimes q_{E}\right) \circ \widehat{\alpha}_{u} .
$$

The $E$-crossed product $A \rtimes_{\alpha, E} G$ "lies between" the maximal and the reduced crossed products and the coaction $\widehat{\alpha}_{u}$ on the full crossed product factors through a coaction $\widehat{\alpha}_{E}$ on $A \rtimes_{\alpha, E} G$ by [15, Theorem 6.2]. We also have $\mathbb{C} \rtimes_{E} G \cong C_{E}^{*}(G)$, which follows from the fact that the comultiplication on $C^{*}(G)$ factors through a coaction $\delta: C_{E}^{*}(G) \rightarrow \mathcal{M}\left(C_{E}^{*}(G) \otimes C^{*}(G)\right)$.

So assume from now on that $E \subseteq B(G)$ is a $G$-invariant weak*-closed nonzero ideal and that $\delta: B \rightarrow \mathcal{M}\left(B \otimes C^{*}(G)\right)$ is any given coaction. By Theorem 4.6 and Corollary 4.7, we know that there exists a canonical coaction $\delta_{E}: B_{E} \rightarrow$ $\mathcal{M}\left(B_{E} \otimes C^{*}(G)\right)$ which satisfies $E$-duality and which has the same dual system as the original coaction $\delta$, i.e., $\left(B_{E}, \delta_{E}\right)$ is an $E$-ization for $(B, \delta)$. We now want to describe the algebra $B_{E}$ in terms of $E$. At the same time, we give a positive answer to [15, Conjecture 6.14]:

Theorem 5.1. Let $\delta_{u}: B_{u} \rightarrow \mathcal{M}\left(B_{u} \otimes C^{*}(G)\right)$ denote the maximalization of the coaction $\delta: B \rightarrow \mathcal{M}\left(B \otimes C^{*}(G)\right)$. Then $B_{E}=B_{u} / J_{\delta, E}$ with

$$
J_{\delta, E}=\operatorname{ker}\left(\operatorname{id}_{B_{u}} \otimes q_{E}\right) \circ \delta_{u} \subseteq B_{u} .
$$

Moreover, the coaction $\delta_{u}$ factors to give a coaction $\delta_{E}: B_{E} \rightarrow \mathcal{M}\left(B_{E} \otimes C^{*}(G)\right)$ which satisfies E-duality. In particular, any dual coaction $\widehat{\alpha}_{E}$ on any E-crossed product $A \rtimes_{\alpha, E} G$ satisfies E-duality.

Proof. Let $A=B \rtimes_{\delta} \widehat{G}$ equipped with the canonical structure of a weakly proper $G \rtimes G$-algebra and let $\left(\mathcal{F}_{u}(A), \delta_{\mathcal{F}_{u}(A)}\right)$ denote the coaction on the $B_{u}-A \rtimes_{\alpha} G$ equivalence bimodule $\mathcal{F}_{u}(A)$ of Theorem 4.6, with $\alpha:=\widehat{\delta}$. The theorem will follow from Theorem 4.6 as soon as we can show that the ideal $J_{\delta, E}$ in $B_{u}$ corresponds to the ideal $J_{\alpha, E}$ in $A \rtimes_{\alpha} G$ under the Rieffel-correspondence. But this follows from the existence of the bimodule map

$$
\left(\operatorname{id}_{\mathcal{F}_{u}(A)} \otimes q_{E}\right) \circ \delta_{\mathcal{F}_{u}(A)}: \mathcal{F}_{u}(A) \rightarrow \mathcal{M}\left(\mathcal{F}_{u}(A) \otimes C_{E}^{*}(G)\right)
$$

which is compatible with $\left(\operatorname{id}_{B_{u}} \otimes q_{E}\right) \circ \delta_{u}$ on the left and $\left(\operatorname{id}_{A \rtimes G} \otimes q_{E}\right) \circ \widehat{\alpha}_{u}$ on the right hand side of the module.

If we apply this result to a dual coaction $\delta_{E}=\widehat{\alpha}_{E}$, we see that all dual coactions on $E$-crossed products satisfy $E$-duality. 
It might be reasonable to ask whether every coaction $\delta: B \rightarrow \mathcal{M}\left(B \otimes C^{*}(G)\right)$ is one of the coactions $\delta_{E}$ for some ideal $E$. By the above theorem, this is actually equivalent to asking whether every dual coaction $\widehat{\alpha}_{\mu}$ on some intermediate crossed product $A \rtimes_{\alpha, \mu} G$ equals $\widehat{\alpha}_{E}$ for some $G$-invariant weak*-closed ideal $E \subseteq B(G)$. We shall see below that this is not the case. For the proof, we first need:

Proposition 5.2. Let $E$ be a $G$-invariant weak*-closed ideal of $B(G)$. Let $\alpha: G \rightarrow$ $\operatorname{Aut}(A)$ and $\beta: G \rightarrow \operatorname{Aut}(B)$ be actions and let $\Theta: A \rightarrow \mathcal{M}(B)$ be a $G$-equivariant *-homomorphism. Then the inclusion $C_{c}(G, A) \rightarrow C_{c}(G, \mathcal{M}(B)) \subseteq \mathcal{M}\left(B \rtimes_{\beta, E} G\right)$ extends to a (unique) *-homomorphism $\Theta \rtimes_{E} G: A \rtimes_{\alpha, E} G \rightarrow \mathcal{M}\left(B \rtimes_{\beta, E} G\right)$.

Proof. Let $\Theta \rtimes_{u} G: A \rtimes_{\alpha} G \rightarrow \mathcal{M}\left(B \rtimes_{\beta, u} G\right)$ denote the corresponding map for the universal norms. We need to show that the ideal $J_{\alpha, E}=\operatorname{ker}\left(\operatorname{id}_{A \rtimes G} \otimes q_{E}\right) \circ \widehat{\alpha}_{u}$ contains $\operatorname{ker}\left(\Theta \rtimes_{u} G\right)$. But this follows from the commutativity of the diagram

$$
\begin{array}{ccc}
A \rtimes_{\alpha} G & \stackrel{\Theta \rtimes_{u} G}{\longrightarrow} & \mathcal{M}\left(B \rtimes_{\beta, u} G\right) \\
\left(\operatorname{id} \otimes q_{E}\right) \widehat{\alpha} \downarrow & \downarrow\left(\operatorname{id} \otimes q_{E}\right) \circ \widehat{\beta} \\
\mathcal{M}\left(A \rtimes_{\alpha} G \otimes C_{E}^{*}(G)\right) \stackrel{\left(\Theta \rtimes_{u} G\right) \otimes \operatorname{id}_{G}}{\longrightarrow} \mathcal{M}\left(B \rtimes_{\beta, u} G \otimes C_{E}^{*}(G)\right)
\end{array}
$$

where we extended maps to multiplier algebras where necessary, which is no problem if $\Theta: A \rightarrow \mathcal{M}(B)$ is assumed to be nondegenerate. In case that $\Theta$ is degenerate, we may replace $\mathcal{M}\left(A \rtimes_{\alpha} G \otimes C_{E}^{*}(G)\right)$ by the subalgebra $\widetilde{M}\left(A \rtimes_{\alpha} G \otimes C_{E}^{*}(G)\right)$ consisting of all $m$ which satisfy $m(1 \otimes z) \in A \rtimes_{\alpha} G \otimes C_{E}^{*}(G)$ for all $z \in C_{E}^{*}(G)$ in the lower left corner of the diagram, on which there always exists a unique extension of $\left(\Theta \rtimes_{u} G\right) \otimes \operatorname{id}_{G}$.

Note that it follows in particular from the above proposition that for all weak-* closed ideals $E \subseteq B(G)$ the morphism $\mathbb{C} \rightarrow \mathcal{M}(A) ; \lambda \mapsto \lambda 1$ induces a canonical map

$$
i_{C_{E}^{*}(G)}: C_{E}^{*}(G)=\mathbb{C} \rtimes_{E} G \rightarrow \mathcal{M}\left(A \rtimes_{\alpha, E} G\right) .
$$

Example 5.3 (Counter-example to Conjecture 6.12 in [15]). Let $G$ be any locally compact group such that $C^{*}(G)$ is not nuclear (e.g. any discrete non-amenable group). Then there exists a $C^{*}$-algebra $A$ such that

$$
A \otimes \max C^{*}(G) \nsucceq A \otimes C^{*}(G) \nsucceq A \otimes C_{r}^{*}(G),
$$

where we understand the symbol $\nRightarrow$ in the sense that the canonical surjective morphisms from left to right are not injective. Consider the trivial action $\operatorname{tr}$ of $G$ on $A$. Then

$$
A \rtimes_{\mathrm{tr}, u} G \cong A \otimes_{\max } C^{*}(G) \text { and } A \rtimes_{\mathrm{tr}, r} G \cong A \otimes C_{r}^{*}(G),
$$

so that the tensor product $A \otimes C^{*}(G)$ can be regarded as a $\mu$-crossed product $A \rtimes_{\operatorname{tr}, \mu} G$ for some crossed-product norm $\|\cdot\|_{\mu}$ lying between $\|\cdot\|_{u}$ and $\|\cdot\|_{r}$. Moreover, $\mathrm{id}_{A} \otimes \delta_{G}$ is a coaction on $A \otimes C^{*}(G)$ which corresponds to the dual coaction $\widehat{\operatorname{tr}}_{\mu}$ under the identification $A \otimes C^{*}(G) \cong A \rtimes_{\operatorname{tr}, \mu} G$.

We claim that $\|\cdot\|_{\mu}$ is not an $E$-norm for any $G$-invariant weak*-closed ideal $E \subseteq B(G)$. Assume to the contrary that $\|\cdot\|_{\mu}=\|\cdot\|_{E}$ for some $E$. Since $\|\cdot\|_{\mu}$ is strictly smaller than $\|\cdot\|_{u}$, we then must have that $C_{E}^{*}(G)$ is a proper quotient of $C^{*}(G)$. By Proposition 5.2 , the canonical (injective) inclusion $i_{C^{*}(G)}: C^{*}(G) \rightarrow$ $\mathcal{M}\left(A \otimes C^{*}(G)\right)$ must factor through a map $i_{C_{E}^{*}(G)}: C_{E}^{*}(G) \rightarrow \mathcal{M}\left(A \otimes C^{*}(G)\right)$. But this is impossible since it forces the surjection $C^{*}(G) \rightarrow C_{E}^{*}(G)$ to be injective.

It follows from Theorem 5.1 that the coaction on $A \otimes C^{*}(G)$ cannot satisfy $E$-duality for any $E$ (although it satisfies duality for some crossed-product norm). This gives a negative answer to [15, Conjecture 6.12]. 


\section{Functoriality}

Throughout this section, we fix a locally compact group $G$ and a $G$-invariant weak-* closed ideal $E$ of $B(G)$ containing $A(G)$ and we denote by $\|\cdot\|_{E}$ the corresponding crossed-product norm on $C_{c}(G, A)$ for any $G$-algebra $(A, \alpha)$. We write $A \rtimes_{\alpha, E} G$ or simply $A \rtimes_{E} G$ for the associated crossed product. Proposition 5.2 shows that such norms have good functorial properties (and it is, in fact, not difficult to see that the $E$-crossed product construction $A \mapsto A \rtimes_{E} G$ yields a functor between suitable categories). We want to show that our constructions are also functorial for such norms. More precisely, we show that the construction $A \mapsto A_{E}^{G}:=A_{E}^{G, \alpha}$ extends to a functor between appropriate categories of $C^{*}$-algebras. We will consider both natural categories of $C^{*}$-algebras with (equivariant) ordinary *-homomorphisms or nondegenerate ${ }^{*}$-homomorphisms into multiplier algebras as their morphisms. In both cases we obtain functoriality in complete generality (the actions here might be even non-saturated).

Remark 6.1. Let $\mathcal{E}_{i}$ be a Hilbert $B_{i}$-module for $i=1,2$ and let $\phi: B_{1} \rightarrow \mathcal{M}\left(B_{2}\right)$ be a (possibly degenerate) ${ }^{*}$-homomorphism. A morphism from $\mathcal{E}_{1}$ to $\mathcal{E}_{2}$ compatible with $\phi$ is a linear map $\psi: \mathcal{E}_{1} \rightarrow \mathcal{M}\left(\mathcal{E}_{2}\right):=\mathcal{L}\left(B_{2}, \mathcal{E}_{2}\right)$ satisfying $\psi(\xi \cdot b)=\psi(\xi) \circ \phi(b)$ and $\phi\left(\langle\xi \mid \eta\rangle_{B_{1}}\right)=\psi(\xi)^{*} \circ \psi(\eta)$ for all $\xi, \eta \in \mathcal{E}_{1}$ and $b \in B_{1}$. Such a morphism induces a ${ }^{*}$-homomorphism $\tilde{\psi}: \mathcal{K}\left(\mathcal{E}_{1}\right) \rightarrow \mathcal{L}\left(\mathcal{E}_{2}\right)=\mathcal{M}\left(\mathcal{K}\left(\mathcal{E}_{2}\right)\right)$ satisfying

$$
\tilde{\psi}\left(\mathcal{K}\left(\mathcal{E}_{1}\right)\langle\xi \mid \eta\rangle\right)=\psi(\xi) \circ \psi(\eta)^{*}
$$

(see [19, Lemma 2.2]). If $\phi$ is injective (i.e., isometric), then so is $\psi$.

Moreover, we say that such $\psi: \mathcal{E}_{1} \rightarrow \mathcal{M}\left(\mathcal{E}_{2}\right)$ is nondegenerate if $\psi\left(\mathcal{E}_{1}\right) B_{2}$ is dense in $\mathcal{E}_{2}$. It follows then that $\tilde{\psi}: \mathcal{K}\left(\mathcal{E}_{1}\right) \rightarrow \mathcal{M}\left(\mathcal{K}\left(\mathcal{E}_{2}\right)\right)$ is also nondegenerate by [12, Example 1.10].

Proposition 6.2. Let $A$ and $B$ be two $X \rtimes G$-algebras and let $\pi: A \rightarrow \mathcal{M}(B)$ be $a$ (possibly degenerate) $X \rtimes G$-equivariant*-homomorphism, that is, a*-homomorphism which intertwines the actions of $G$ and $C_{0}(X)$ on $A$ and $B$. Then $\pi$ induces a morphism $\mathcal{F}_{E}(\pi): \mathcal{F}_{E}(A) \rightarrow \mathcal{M}\left(\mathcal{F}_{E}(B)\right)=\mathcal{L}\left(B \rtimes_{E} G, \mathcal{F}_{E}(B)\right)$ of Hilbert modules which is compatible with the homomorphism $\pi \rtimes_{E} G: A \rtimes_{E} G \rightarrow \mathcal{M}\left(B \rtimes_{E} G\right.$ ) (from Proposition 5.2) and which is given by the formula:

$$
\mathcal{F}_{E}(\pi)(\xi) g=\pi(\xi) * g=\int_{G} \Delta(t)^{-1 / 2} \beta_{t}\left(\pi(\xi) g\left(t^{-1}\right)\right) d t
$$

for all $\xi \in \mathcal{F}_{c}(A)=C_{c}(X) \cdot A$ and $g \in C_{c}(G, B)$. Moreover, $\pi$ induces a ${ }^{*}$-homomorphism $\pi_{E}^{G}: A_{E}^{G} \rightarrow \mathcal{M}\left(B_{E}^{G}\right)$ satisfying $\pi_{E}^{G}\left(\mathbb{E}^{\alpha}(a)\right)=\mathbb{E}^{\beta}(\pi(a))$ for all a $\in A_{c}=$ $C_{c}(X) \cdot A \cdot C_{c}(X)$. If $\pi \rtimes_{E} G$ is injective or nondegenerate (the latter follows, if $\pi$ is nondegenerate), then so are $\mathcal{F}_{E}(\pi)$ and $\pi_{E}^{G}$.

Proof. The ${ }^{*}$-homomorphism $\pi \rtimes_{E} G$ induces a compatible morphism

$$
\operatorname{id}_{\mathcal{F}(X)} \otimes \pi \rtimes_{E} G: \mathcal{F}(X) \otimes_{C_{0}(X) \rtimes G}\left(A \rtimes_{E} G\right) \rightarrow \mathcal{M}\left(\mathcal{F}(X) \otimes_{C_{0}}(X) \rtimes G\left(B \rtimes_{E} G\right)\right)
$$

which maps $f \otimes h \in C_{c}(X) \otimes C_{c}(G, A)$ to $f \otimes\left(\pi \rtimes_{E} G\right)(h)$. Under the canonical isomorphisms $\mathcal{F}(X) \otimes_{C_{0}(X) \rtimes G}\left(A \rtimes_{E} G\right) \cong \mathcal{F}_{E}(A)$ and $\mathcal{F}(X) \otimes_{C_{0}(X) \rtimes G}\left(B \rtimes_{E} G\right) \cong$ $\mathcal{F}_{E}(B)$ this therefore induces a compatible morphism $\mathcal{F}_{E}(\pi): \mathcal{F}_{E}(A) \rightarrow \mathcal{M}\left(\mathcal{F}_{E}(B)\right)$ and the description of the above decompositions for $\mathcal{F}_{E}(A)$ and $\mathcal{F}_{E}(B)$ in (2.10) together with a straightforward computation shows that $\mathcal{F}_{E}(\pi)$ is given by (6.3). By the above remark, $\mathcal{F}_{E}(\pi)$ induces a *-homomorphism $\pi_{E}^{G}: A_{E}^{G}=\mathcal{K}\left(\mathcal{F}_{E}(A)\right) \rightarrow$ $\mathcal{M}\left(\mathcal{K}\left(\mathcal{F}_{E}(B)\right)\right)=\mathcal{M}\left(B_{E}^{G}\right)$ determined by $\pi_{E}^{G}(\mathcal{K}\langle\xi \mid \eta\rangle)=\mathcal{F}_{E}(\pi)(\xi) \circ \mathcal{F}_{E}(\pi)(\eta)^{*}$ for all $\xi, \eta \in \mathcal{F}_{c}(A)$. Now, the adjoint operator $\mathcal{F}_{E}(\pi)(\eta)^{*}$ is easily seen to be given by

$$
\left.\mathcal{F}_{E}(\pi)(\eta)^{*} \zeta\right|_{t}=\langle\langle\pi(\eta) \mid \zeta\rangle\rangle_{C_{c}(G, B)}(t)=\Delta(t)^{-1 / 2} \pi(\eta)^{*} \beta_{t}(\zeta)
$$


for all $\zeta \in \mathcal{F}_{c}(B)$. Hence, for $a=\xi \eta^{*} \in A_{c}$, we get

$$
\begin{aligned}
\pi^{G}\left(\mathbb{E}^{\alpha}(a)\right) \zeta=\pi_{E}^{G}(\mathcal{K}\langle\xi \mid \eta\rangle) \zeta=\mathcal{F}_{E}(\pi)(\xi) \circ \mathcal{F}_{E}(\pi)(\eta)^{*} \zeta \\
=\pi(\xi) *\langle\langle\pi(\eta) \mid \zeta\rangle\rangle_{C_{c}(G, B)}=\mathbb{E}^{\beta}\left(\pi\left(\xi \eta^{*}\right)\right) \zeta=\mathbb{E}^{\beta}(\pi(a)) \zeta .
\end{aligned}
$$

The last assertion concerning injectivity follows from Remark 6.1] The assertion about nondegeneracy follows from the fact that if $\pi$ is nondegenerate, then so is $\pi \rtimes_{E} G$ and hence also $\operatorname{id}_{\mathcal{F}(X)} \otimes \pi \rtimes_{E} G \cong \mathcal{F}_{E}(\pi)$ and its induced homomorphism $\pi^{G}$ on compact operators.

Remark 6.4. (1) Given $x \in C_{c}(X) \cdot \mathcal{M}(B)$ (for a weakly proper $X \rtimes G$-algebra $B$ ), notice that $x$ may be viewed as an element of $\mathcal{M}\left(\mathcal{F}_{E}(B)\right)$ (that is, a multiplier of the Hilbert module $\left.\mathcal{F}_{E}(B)\right)$ by the formula $x \cdot g:=\int_{G} \Delta(t)^{-1 / 2} \beta_{t}\left(x g\left(t^{-1}\right)\right) d t$ (and adjoint given by $\left.x^{*} \zeta\right|_{t}=\Delta(t)^{-1 / 2} x^{*} \beta_{t}(\zeta)$ for all $\zeta \in \mathcal{F}_{c}(B)$ ). In this way, the formula (6.3) for the morphism $\mathcal{F}_{E}(\pi)$ can be described more easily as $\mathcal{F}_{E}(\pi)(\xi)=$ $\pi(\xi)$ for all $\xi \in \mathcal{F}_{c}(A)$, i.e., the map $\mathcal{F}_{E}(\pi)$ is essentially $\pi$ when restricted to $\mathcal{F}_{c}(A) \subseteq A$. The homomorphism $\pi_{E}^{G}: A_{E}^{G} \rightarrow \mathcal{M}\left(B_{E}^{G}\right)$ may also be interpreted similarly. In fact, if $\pi$ is nondegenerate, then $\pi_{E}^{G}\left(\mathbb{E}^{\alpha}(a)\right)=\mathbb{E}^{\beta}(\pi(a))=\pi\left(\mathbb{E}^{\alpha}(a)\right)$ for all $a \in A_{c}$, so that $\pi_{E}^{G}$ coincides with the extension of $\pi$ to $\mathcal{M}(A)$ on $A_{c}^{G} \subseteq \mathcal{M}(A)$. In general, for a possibly degenerate ${ }^{*}$-homomorphism $\pi: A \rightarrow \mathcal{M}(B)$, we can consider the bidual von Neumann algebras $A^{\prime \prime}$ and $B^{\prime \prime}$ and the weakly continuous extension $\pi^{\prime \prime}: A^{\prime \prime} \rightarrow B^{\prime \prime}$ of $\pi$. Then $\mathbb{E}^{\beta}(\pi(a))=\pi^{\prime \prime}\left(\mathbb{E}^{\alpha}(a)\right)$ for all $a \in A_{c}$ because $\mathbb{E}^{\alpha}(a)=\int_{G}^{\text {st }} \alpha_{t}(a) d t$ may be interpreted as a weak limit in $\mathcal{M}(A) \subseteq A^{\prime \prime}$. Therefore we can still say that $\pi_{E}^{G}$ is the restriction of $\pi^{\prime \prime}$ to $A_{c}^{G}$.

(2) For nondegenerate $G$-equivariant homomorphisms $\pi: A \rightarrow \mathcal{M}(B)$ and reduced norms $\left(i . e\right.$., for $E=B_{r}(G)$ ), the existence of $\pi_{r}^{G}: A_{r}^{G} \rightarrow \mathcal{M}\left(B_{r}^{G}\right)$ was already obtained in [18, Proposition 2.6] using a more direct approach (which makes the proof much more technical and involved). The proof in this case could have been done also by using the above idea and the tensor decomposition $\mathcal{F}_{r}(A) \cong$ $\mathcal{F}(X) \otimes_{C_{0}(X) \rtimes G}\left(A \rtimes_{r} G\right)$ already available from [23, Theorem 7.1].

The above result already indicates that the assignment $A \mapsto A_{E}^{G}$ is a functor between two different types of $C^{*}$-categories. To be more precise, for a fixed locally compact group and a $G$-space $X$, let us consider the categories $\mathfrak{C}^{*}(X, G)$ and $\mathfrak{C}_{\text {nd }}^{*}(X, G)$ whose objects (in both) are weak $X \rtimes G$-algebras and whose morphisms $A \rightarrow B$ in $\mathfrak{C}^{*}(X, G)$ are $X \rtimes G$-equivariant *-homomorphisms from $A$ into $B$, and in $\mathfrak{C}_{\mathrm{nd}}^{*}(X, G)$ are $X \rtimes G$-equivariant nondegenerate ${ }^{*}$-homomorphism $A \rightarrow \mathcal{M}(B)$. In particular, considering the trivial group $\{e\}$ and a space $Y$, we may consider the categories $\mathfrak{C}^{*}(Y):=\mathfrak{C}^{*}(Y,\{e\})$ and $\mathfrak{C}_{\text {nd }}^{*}(Y):=\mathfrak{C}^{*}(Y,\{e\})$ consisting of weak $Y$-algebras (i.e., $C^{*}$-algebras $A$ endowed with a nondegenerate ${ }^{*}$-homomorphism $\left.C_{0}(Y) \rightarrow \mathcal{M}(A)\right)$ and whose morphisms are either $Y$-equivariant *-homomorphisms $A \rightarrow B$ or nondegenerate $Y$-equivariant *-homomorphisms $A \rightarrow \mathcal{M}(B)$. In the special case that $Y=\{\mathrm{pt}\}$ is the one-point space, we get the ordinary categories $\mathfrak{C}^{*}$ and $\mathfrak{C}_{\text {nd }}^{*}$ of $C^{*}$-algebras with *-homomorphisms or nondegenerate ${ }^{*}$-homomorphisms as their morphisms.

Corollary 6.5. If $G$ acts properly on a space $X$ and $\|\cdot\|_{E}$ denotes a $C^{*}$-norm coming from a nonzero $G$-invariant weak-*-closed ideal $E \subseteq B(G)$, then the assignments $A \mapsto A_{E}^{G}$ and $\pi \mapsto \pi_{E}^{G}$ constructed above yield functors $\mathfrak{C}^{*}(X, G) \rightarrow \mathfrak{C}^{*}(G \backslash X)$ and $\mathfrak{C}_{\text {nd }}^{*}(X, G) \rightarrow \mathfrak{C}_{\text {nd }}^{*}(G \backslash X)$.

Proof. For a $X \rtimes G$-equivariant ${ }^{*}$-homomorphism $\pi: A \rightarrow B$ it is clear that $\mathcal{F}_{E}(\pi)$ maps $\mathcal{F}(A)$ into $\mathcal{F}(B)$ and hence that $\pi^{G}$ maps $A_{E}^{G}$ into $B_{E}^{G}$. It is also clear that these maps are $G \backslash X$-equivariant. An easy exercise shows that $\pi \mapsto \mathcal{F}_{E}(\pi)$ (and hence also $\left.\pi \mapsto \pi_{E}^{G}\right)$ respects composition, i.e., $\mathcal{F}_{E}(\pi \circ \rho)=\mathcal{F}_{E}(\pi) \circ \mathcal{F}_{E}(\rho)$. This 
yields the functor $\mathfrak{C}^{*}(X, G) \rightarrow \mathfrak{C}^{*}(G \backslash X)$ and the functor $\mathfrak{C}_{\text {nd }}^{*}(X, G) \rightarrow \mathfrak{C}_{\text {nd }}^{*}(G \backslash X)$ is obtained similarly.

\section{Categorical Landstad Duality}

In this section we interpret our main result on Landstad duality (Theorem4.6) in categorical terms extending to exotic norms one of the main results by Kaliszewski, Quigg and Raeburn in [18] for reduced generalized fixed-point algebras.

For a fixed locally compact group $G$, we let $G$ act on it self by right translation and consider the categories $\mathfrak{C}^{*}(G, G)$ and $\mathfrak{C}_{\mathrm{nd}}^{*}(G, G)$ (already considered in the previous section). Both categories have the same objects, namely, weak $G \rtimes G$-algebras, the only difference between them are their morphisms which are either $G \rtimes G$-equivariant *-homomorphisms or nondegenerate homomorphisms into multiplier algebras.

Dually, we consider the categories $\mathfrak{C}^{*}(\widehat{G})$ and $\mathfrak{C}_{\text {nd }}^{*}(\widehat{G})$ whose objects are (in both) $\widehat{G}$-algebras, that is, pairs $(B, \delta)$ consisting of a $C^{*}$-algebra $B$ and a $G$-coaction $\delta$ on it. The morphisms in $\mathfrak{C}^{*}(\widehat{G})$ are $\widehat{G}$-equivariant ${ }^{*}$-homomorphisms and in $\mathfrak{C}_{\text {nd }}^{*}(\widehat{G})$ are nondegenerate $\widehat{G}$-equivariant *-homomorphisms into multiplier algebras, where $\widehat{G}$-equivariance of a *-homomorphism between two $\widehat{G}$-algebras means that it commutes with the underlying coactions in the usual sense (see [11 for details).

As observed before, for a given $\widehat{G}$-algebra, the crossed product $B \rtimes_{\delta} \widehat{G}$ carries a canonical structure as a $G \rtimes G$-algebra given by the dual $G$-action and the canonical $C_{0}(G)$-embedding $j_{C_{0}(G)}$ into $\mathcal{M}\left(B \rtimes_{\delta} \widehat{G}\right)$. Moreover, it is well-known (see [11] for further details) that the crossed product construction $(B, \delta) \mapsto B \rtimes_{\delta} \widehat{G}$ may be viewed as a functor $\mathrm{CP}_{\widehat{G}}$ between the categories $\mathfrak{C}^{*}(\widehat{G}) \rightarrow \mathfrak{C}^{*}(G, G)$ and $\mathfrak{C}_{\text {nd }}^{*}(\widehat{G}) \rightarrow$ $\mathfrak{C}_{\mathrm{nd}}^{*}(G, G)$.

From now on, we fix a crossed-product norm $\|\cdot\|_{E}$ associated to a nonzero $G$-invariant ideal $E \subseteq B(G)$. For such an $E$, we are interested in the full subcategories $\mathfrak{C}^{*}(\widehat{G})_{E}$ and $\mathfrak{C}_{\mathrm{nd}}^{*}(\widehat{G})_{E}$ of $\mathfrak{C}^{*}(\widehat{G})$ and $\mathfrak{C}_{\mathrm{nd}}^{*}(\widehat{G})$, whose objects are $E$-coactions as defined in Definition 4.5 .

Given a weak $G \rtimes G$-algebra $(A, \alpha)$, Theorem 4.6 implies that the $E$-generalized fixed-point algebra $A_{E}^{G}$ carries an $E$-coaction $\delta_{E}$, that is, it may be viewed as an object of $\mathfrak{C}^{*}(\widehat{G})_{E}$ or $\mathfrak{C}_{\text {nd }}^{*}(\widehat{G})_{E}$. Moreover, we already know that $\operatorname{Fix}_{E}^{G}$ may be viewed as a functor $\mathfrak{C}^{*}(G, G) \rightarrow \mathfrak{C}^{*}$ or $\mathfrak{C}_{\mathrm{nd}}^{*}(G, G) \rightarrow \mathfrak{C}_{\mathrm{nd}}^{*}$. We want to view $\operatorname{Fix}_{\mu}^{G}$ as a functor $\mathfrak{C}^{*}(G, G) \stackrel{\sim}{\rightarrow} \mathfrak{C}^{*}(\widehat{G})_{E}$ or $\mathfrak{C}_{\mathrm{nd}}^{*}(G, G) \stackrel{\sim}{\rightarrow} \mathfrak{C}_{\mathrm{nd}}^{*}(\widehat{G})_{E}$. For this the only missing point is to see that Fix $_{E}^{G}$ is well-defined on the level of morphisms, i.e., if $\pi$ is a morphism from $A$ to $B$ in $\mathfrak{C}^{*}(G, G)$ or $\mathfrak{C}_{\mathrm{nd}}^{*}(G, G)$, then the induced morphism $\pi_{E}^{G}$ from $A_{E}^{G}$ to $B_{E}^{G}$ (in $\mathfrak{C}^{*}$ or $\mathfrak{C}_{\mathrm{nd}}^{*}$ ) is $\widehat{G}$-equivariant and hence a morphism in $\mathfrak{C}^{*}(\widehat{G})_{E}$ or $\mathfrak{C}_{\mathrm{nd}}^{*}(\widehat{G})_{E}$. This will follow from the following lemma:

Lemma 7.1. If $A$ and $B$ are weak $G \rtimes G$-algebras and $\pi: A \rightarrow \mathcal{M}(B)$ is a $G \rtimes G$-equivariant ${ }^{*}$-homomorphism, then the induced ${ }^{*}$-homomorphism $\pi_{E}^{G}: A_{E}^{G} \rightarrow$ $\mathcal{M}\left(B_{E}^{G}\right)$ is $\widehat{G}$-equivariant.

Proof. By construction (see the proof of Proposition 6.2), the homomorphism $\pi_{E}^{G}: A_{E}^{G} \rightarrow \mathcal{M}\left(B_{E}^{G}\right)$ is induced on the algebras of compact operators by the morphism $\tilde{\pi}:=\mathcal{F}_{E}^{G}(\pi): \mathcal{F}_{E}^{G}(A) \rightarrow \mathcal{M}\left(\mathcal{F}_{E}^{G}(B)\right)$ given as in Proposition 6.2, and the coactions on $A_{E}^{G}$ and $B_{E}^{G}$ are induced by the corresponding $G$-coactions $\delta_{\mathcal{F}_{E}^{G}(A)}$ on $\mathcal{F}_{E}^{G}(A)$ and $\delta_{\mathcal{F}_{E}^{G}(B)}$ on $\mathcal{F}_{E}^{G}(B)$ as defined in Lemma 4.12, Hence, it is enough to see that $\mathcal{F}(\pi)$ is $\widehat{G}$-equivariant with respect to the $G$-coactions $\delta_{\mathcal{F}_{E}^{G}(A)}$ and $\delta_{\mathcal{F}_{E}^{G}(B)}$, i.e., it is enough to show that

$$
(\tilde{\pi} \otimes \mathrm{id})\left(\delta_{\mathcal{F}_{E}^{G}(A)}(\xi)\right)=\delta_{\mathcal{F}_{E}^{G}(B)}(\tilde{\pi}(\xi)) \quad \text { for all } \xi \in \mathcal{F}_{c}^{G}(A) .
$$


For this we use the formula $\delta_{\mathcal{F}_{E}^{G}(A)}(\xi)=\left(\phi_{A} \otimes \mathrm{id}\right)\left(\omega_{G}\right)(\xi \otimes 1)$ for the coaction $\delta_{\mathcal{F}_{E}^{G}(A)}$, where $\phi_{A}$ denotes the structural map $C_{0}(G) \rightarrow \mathcal{M}(A)$. As in the proof of Lemma 4.12, the element $\left(\phi_{A} \otimes \mathrm{id}\right)\left(\omega_{G}\right)(\xi \otimes 1)$ is (rigorously) in $\mathcal{M}\left(A \otimes C^{*}(G)\right)$, but is actually interpreted as an element in $\mathcal{M}\left(\mathcal{F}_{E}^{G}(A) \otimes C^{*}(G)\right)$. Similarly, we have the formula $\delta_{\mathcal{F}_{G}^{G(B)}}(\eta)=\left(\phi_{B} \otimes \mathrm{id}\right)\left(\omega_{G}\right)(\eta \otimes 1)$ for all $\eta \in \mathcal{F}_{c}^{G}(B)$. Moreover, this same formula (with a similar interpretation) also holds for $\eta \in \phi_{B}\left(C_{c}(G)\right) \mathcal{M}(B)$, so that we may also apply it for $\eta=\tilde{\pi}(\xi)=\pi(\xi)$ (using the interpretation for $\tilde{\pi}=\mathcal{F}_{E}^{G}(\pi)$ given in Remark 6.4). Using this and the equivariance of $\pi$ with respect to the $C_{0}(G)$-homomorphisms $\phi_{A}$ and $\phi_{B}$ (that is, the fact that $\pi\left(\phi_{A}(f) a\right)=\phi_{B}(f) \pi(a)$ for all $a \in A$ ), we get, for every element $z \in C^{*}(G)$,

$$
\begin{aligned}
(\tilde{\pi} \otimes \mathrm{id})\left(\delta_{\mathcal{F}_{E}^{G}(A)}(\xi)\right)(1 \otimes z) & =(\pi \otimes \mathrm{id})\left(\phi_{A} \otimes \mathrm{id}\left(\omega_{G}\right)(\xi \otimes z)\right) \\
& =\left(\phi_{B} \otimes \mathrm{id}\right)\left(\omega_{G}\right)(\pi(\xi) \otimes z)=\delta_{\mathcal{F}_{E}^{G}(B)}(\tilde{\pi}(\xi))(1 \otimes z) .
\end{aligned}
$$

Since $z \in C^{*}(G)$ was arbitrary, this yields the desired $\widehat{G}$-equivariance of $\tilde{\pi}$.

The above lemma implies that the assignment $\operatorname{Fix}_{E}^{G}$ which maps $A$ to $A_{E}^{G}$ and a morphism $\pi$ from $A$ to $B$ to $\pi_{E}^{G}$ may be viewed as a functor $\mathfrak{C}^{*}(G, G) \rightarrow \mathfrak{C}^{*}(\widehat{G})_{E}$ or $\mathfrak{C}_{\text {nd }}^{*}(G, G) \rightarrow \mathfrak{C}_{\text {nd }}^{*}(\widehat{G})_{E}$. The following result extends to exotic generalized fixedpoint algebras some results which have been obtained in [18, Theorem 4.2] in the case of reduced generalized fixed-point algebras:

Theorem 7.2 (Categorical Landstad Duality). For a locally compact group $G$ and a crossed-product norm $\|\cdot\|_{E}$ associated to a nonzero $G$-invariant ideal $E \subseteq$ $B(G)$, the functor $\operatorname{Fix}_{E}^{G}:(A, \alpha) \mapsto\left(A_{E}^{G}, \delta_{E}\right)$ is an equivalence between the categories $\mathfrak{C}^{*}(G, G) \stackrel{\sim}{\longrightarrow} \mathfrak{C}^{*}(\widehat{G})_{E}$ and $\mathfrak{C}_{\mathrm{nd}}^{*}(G, G) \stackrel{\sim}{\longrightarrow} \mathfrak{C}_{\mathrm{nd}}^{*}(\widehat{G})_{E}$. The crossed product functor $\mathrm{CP}_{\widehat{G}}:(B, \delta) \mapsto B \rtimes_{\delta} \widehat{G}$ is a quasi-inverse functor of $\mathrm{Fix}_{E}^{G}$.

Proof. Given a weak $G \rtimes G$-algebra $(A, \alpha)$, Theorem 4.6 implies that the $E$-generalized fixed-point algebra $A_{E}^{G}$ carries an $E$-coaction $\delta_{E}$ of $G$ and there is a canonical $G \rtimes G$-equivariant isomorphism $A_{E}^{G} \rtimes_{\delta_{E}} \widehat{G} \stackrel{\sim}{\longrightarrow} A$ which is given as the integrated form $\kappa_{A} \rtimes \phi_{A}$ of the covariant representation $\left(\kappa_{A}, \phi_{A}\right):\left(A_{E}^{G}, C_{0}(G)\right) \rightarrow \mathcal{M}(A)$, where $\kappa_{A}: A_{E}^{G} \rightarrow \mathcal{M}(A)$ is the extension of the inclusion map $A_{c}^{G} \hookrightarrow \mathcal{M}(A)$ (i.e., the representation given in Proposition 3.5) and $\phi_{A}: C_{0}(G) \rightarrow \mathcal{M}(A)$ denotes the structural $C_{0}(G)$-homomorphism of the $G \rtimes G$-algebra $A$. Moreover, Theorem 4.6 also says that given an $E$-coaction $(B, \delta)$, there is an isomorphism $\left(A_{E}^{G}, \delta_{E}\right) \cong(B, \delta)$. This means that the functor $\operatorname{Fix}_{E}^{G}$ is essentially surjective (between each of the above pairs of categories). To prove that $\operatorname{Fix}_{E}^{G}$ is an equivalence, it is enough to show that it is full and faithful, i.e., for each pair of weak $G \rtimes G$-algebras $(A, \alpha)$ and $(B, \beta), G \rtimes G$-equivariant morphisms $(A, \alpha) \rightarrow(B, \beta)$ correspond bijectively to $\widehat{G}$-equivariant morphisms $\left(A_{E}^{G}, \delta_{E}^{A}\right) \rightarrow\left(B_{E}^{G}, \delta_{E}^{B}\right)$ via the maps on morphisms induced by the functors $\operatorname{Fix}_{E}^{G}$ and $\mathrm{CP}_{\widehat{G}}$, i.e., the map $\operatorname{Mor}(A, B) \rightarrow \operatorname{Mor}\left(A_{E}^{G}, B_{E}^{G}\right)$ sending $\pi$ to $\pi_{E}^{G}$ is a bijection, where $\operatorname{Mor}(x, y)$ denotes the set of morphisms $x \rightarrow y$ between objects of the underlying category (which in our case can be any one of the categories appearing in the statement). We prove this at the same time for both pairs of categories involving ordinary (equivariant) *-homomorphisms or nondegenerate *-homomorphisms into multiplier algebras as their morphisms. For this let $\pi: A \rightarrow \mathcal{M}(B)$ be a (possibly degenerate) $G \rtimes G$-equivariant *-homomorphism. The induced homomorphism $\pi_{E}^{G}: A_{E}^{G} \rightarrow \mathcal{M}\left(B_{E}^{G}\right)$ is given by $\pi_{E}^{G}\left(\mathbb{E}_{A}(a)\right)=\mathbb{E}_{B}(\pi(a))$ for all $a \in A_{c}$ (see Proposition 6.2), where $\mathbb{E}_{A}(a)=\int_{G}^{\text {st }} \alpha_{t}(a) d t$ and similarly for 
$\mathbb{E}_{B}$. We now prove that the following diagram commutes:

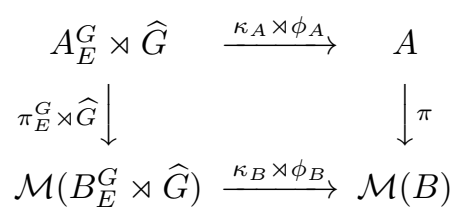

In fact, this follows from the following computation: for all $a \in A_{c}$ and $f \in C_{c}(X)$ we get

$$
\begin{aligned}
\pi\left(\left(\kappa_{A} \rtimes \phi_{A}\right)\left(j_{A_{E}^{G}}\left(\mathbb{E}_{A}(a)\right) j_{G}^{A_{E}^{G}}(f)\right)\right. & =\pi\left(\mathbb{E}_{A}(a) \phi_{A}(f)\right)=\mathbb{E}_{B}(\pi(a)) \phi_{B}(f) \\
& =\left(\kappa_{B} \rtimes \phi_{B}\right)\left(j_{B_{E}^{G}}\left(\mathbb{E}_{B}(\pi(a))\right) j_{G}^{B_{E}^{G}}(f)\right) \\
& =\left(\kappa_{B} \rtimes \phi_{B}\right)\left(\pi_{E}^{G} \rtimes \widehat{G}\right)\left(j_{A_{E}^{G}}(\mathbb{E}(a)) j_{G}^{A_{E}^{G}}(f)\right),
\end{aligned}
$$

where $j_{G}$ is an abbreviation for $j_{C_{0}(G)}$. The commutativity of the above diagram means that $\pi: A \rightarrow \mathcal{M}(B)$ is determined by $\pi_{E}^{G}$ via the canonical isomorphisms $\kappa_{A} \rtimes \phi_{A}: A_{E}^{G} \rtimes \widehat{G} \stackrel{\sim}{\longrightarrow} A$ and $\kappa_{B} \rtimes \phi_{B}: B_{E}^{G} \rtimes \widehat{G} \stackrel{\sim}{\longrightarrow} B$. More precisely, this means that the canonical map $\operatorname{Mor}(A, B) \rightarrow \operatorname{Mor}\left(A_{E}^{G}, B_{E}^{G}\right)$ which sends $\pi$ to $\pi_{E}^{G}$ is injective. It is also surjective because given a (possibly degenerate) $\widehat{G}$-equivariant *-homomorphism $\sigma: A_{E}^{G} \rightarrow \mathcal{M}\left(B_{E}^{G}\right)$, we can consider the corresponding *-homomorphism $\sigma \rtimes \widehat{G}: A_{E}^{G} \rtimes \widehat{G} \rightarrow \mathcal{M}\left(B_{E}^{G} \rtimes \widehat{G}\right)$ which is $G \rtimes G$-equivariant. Composing $\sigma \rtimes \widehat{G}$ with the canonical isomorphisms $\kappa_{A} \rtimes \phi_{A}$ and $\left(\kappa_{B} \rtimes \phi_{B}\right)^{-1}$, this yields a $G \rtimes G$-equivariant homomorphism $\pi:=\left(\kappa_{B} \rtimes \phi_{B}\right)^{-1} \circ(\sigma \rtimes \widehat{G}) \circ \kappa_{A} \rtimes \phi_{A}$ such that $\pi_{E}^{G}=\sigma$ (by the above commutative diagram). Therefore $\operatorname{Fix}_{E}^{G}$ yields an equivalence of categories $\mathfrak{C}^{*}(G, G) \stackrel{\sim}{\longrightarrow} \mathfrak{C}^{*}(\widehat{G})_{E}$ and $\mathfrak{C}_{\text {nd }}^{*}(G, G) \stackrel{\sim}{\rightarrow} \mathfrak{C}_{\text {nd }}^{*}(\widehat{G})_{E}$. Moreover, our arguments above show that the composition functor $\mathrm{CP}_{\widehat{G}} \circ \mathrm{Fix}_{E}^{G}$ is naturally isomorphic to the identity functor on $\mathfrak{C}^{*}(G, G)$ or $\mathfrak{C}_{\text {nd }}^{*}(G, G)$ : the natural isomorphism at a given object (i.e., a weak $G \rtimes G$-algebra) $A$ is the canonical isomorphism $\kappa_{A} \rtimes \phi_{A}: A_{E}^{G} \rtimes \widehat{G} \stackrel{\sim}{\longrightarrow} A$ (notice that the arguments above show that this is natural). This means that $\mathrm{CP}_{\widehat{G}}$ is a left quasi-inverse for $\mathrm{Fix}_{E}^{G}$. Since we already know that Fix $_{E}^{G}$ is an equivalence, it follows that $\mathrm{CP}_{\widehat{G}}$ is a quasi-inverse for $\mathrm{Fix}_{E}^{G}$ (and hence also an equivalence functor).

Remark 7.3. In this paper we did not consider functoriality of our generalized fixedpoint algebra constructions for (equivariant) categories based on correspondences (as done in [3] for the reduced case), but it is indeed possible to obtain functoriality also in this setting (and we postpone the full proof of this fact to a forthcoming paper) under the assumption that all actions involved are saturated (which includes the important case where the action on the underlying proper $G$-space $X$ is free - this is the assumption made in [3] - and, in particular, covers the case of $G \rtimes$ $G$-algebras). We are not going to discuss this, but it is certainly also possible to prove a version of the categorical Landstad duality theorem above for categories of weak $G \rtimes G$ - and $\widehat{G}$-algebras with equivariant correspondences as their morphisms.

\section{REFERENCES}

[1] Claire Anantharaman-Delaroche, On tensor products of group $C^{*}$-algebras and related topics, Limits of graphs in group theory and computer science, 2009, pp. 1-35. MR 2562137

[2] Astrid an Huef, Steven P. Kaliszewski, Iain Raeburn, and Dana P. Williams, Naturality of Rieffel's Morita equivalence for proper actions, Algebr. Represent. Theory 14 (2011), no. 3, 515-543, DOI 10.1007/s10468-009-9201-2. MR 2785921

[3] Astrid an Huef, Iain Raeburn, and Dana P. Williams, Functoriality of Rieffel's generalised fixed-point algebras for proper actions, Superstrings, geometry, topology, and $C^{*}$-algebras, 2010, pp. 9-25. MR 2681756 
[4] _ A symmetric imprimitivity theorem for commuting proper actions, Canad. J. Math. 57 (2005), no. 5, 983-1011. MR 2164592

[5] - Proper actions on imprimitivity bimodules and decompositions of Morita equivalences, J. Funct. Anal. 200 (2003), no. 2, 401-428, DOI 10.1016/S0022-1236(02)00005-8. MR 1979017

[6] Nathanial P. Brown and Erik P. Guentner, New $\mathrm{C}^{*}$-completions of discrete groups and related spaces, Bull. Lond. Math. Soc. 45 (2013), no. 6, 1181-1193, DOI 10.1112/blms/bdt044.

[7] Alcides Buss, A generalized Fourier inversion theorem, Bull. Braz. Math. Soc. (N.S.) 39 (2008), no. 4, 555-571, DOI 10.1007/s00574-008-0004-6. MR 2465264

[8] Alcides Buss and Siegfried Echterhoff, Imprimitivity theorems for weaky proper actions and duality (2013), eprint. arXiv: 1305.5100, to appear in Ergodic theory \& dynamical Systems.

[9] - Weakly proper group actions, Mansfield's imprimitivity and twisted Landstad duality (2013), eprint. arXiv: 1310.3934 to appear in Trans. Amer. Math. Soc.

[10] Siegfried Echterhoff, Steven P. Kaliszewski, and John Quigg, Maximal coactions, Internat. J. Math. 15 (2004), no. 1, 47-61, DOI 10.1142/S0129167X04002107. MR 2039211

[11] Siegfried Echterhoff, Steven P. Kaliszewski, John Quigg, and Iain Raeburn, A categorical approach to imprimitivity theorems for $C^{*}$-dynamical systems, Mem. Amer. Math. Soc. 180 (2006), no. 850, viii+169. MR 2203930

[12] Siegfried Echterhoff and Iain Raeburn, Multipliers of imprimitivity bimodules and Morita equivalence of crossed products, Math. Scand. 76 (1995), no. 2, 289-309. MR 1354585

[13] Robert Fischer, Maximal coactions of quantum groups (2004), SFB 478 Geometrische Strukturen in der Mathematik, Münster, preprint no. 350,.

[14] Philip Green, $C^{*}$-algebras of transformation groups with smooth orbit space, Pacific J. Math. 72 (1977), no. 1, 71-97. MR 0453917

[15] Steven P. Kaliszewski, Magnus B. Landstad, and John Quigg, Exotic group C*-algebras in noncommutative duality, New York J. Math. 19 (2013), 689-711.

[16] Steven P. Kaliszewski, Paul S. Muhly, John Quigg, and Dana P. Williams, Fell bundles and imprimitivity theorems: towards a universal generalized fixed point algebra (2012), preprint. arXiv: 1206.6739

[17] Steven P. Kaliszewski and John Quigg, Categorical Landstad duality for actions, Indiana Univ. Math. J. 58 (2009), no. 1, 415-441. MR 2504419

[18] Steven P. Kaliszewski, John Quigg, and Iain Raeburn, Proper actions, fixed-point algebras and naturality in nonabelian duality, J. Funct. Anal. 254 (2008), no. 12, 2949-2968, DOI 10.1016/j.jfa.2008.03.010. MR 2418615

[19] Tsuyoshi Kajiwara, Claudia Pinzari, and Yasuo Watatani, Ideal structure and simplicity of the $C^{*}$-algebras generated by Hilbert bimodules, J. Funct. Anal. 159 (1998), no. 2, 295-322, DOI 10.1006/jfan.1998.3306. MR 1658088

[20] Gennadi G. Kasparov, Equivariant KK-theory and the Novikov conjecture, Invent. Math. 91 (1988), no. 1, 147-201, DOI 10.1007/BF01404917. MR 918241

[21] Yoshikazu Katayama, Takesaki's duality for a nondegenerate co-action, Math. Scand. 55 (1984), no. 1, 141-151. MR 769030

[22] Eberhard Kirchberg, On nonsemisplit extensions, tensor products and exactness of group $C^{*}$-algebras, Invent. Math. 112 (1993), no. 3, 449-489, DOI 10.1007/BF0123244MR 1218321

[23] Ralf Meyer, Generalized fixed point algebras and square-integrable groups actions, J. Funct. Anal. 186 (2001), no. 1, 167-195, DOI 10.1006/jfan.2001.3795. MR 1863296

[24] John C. Quigg, Landstad duality for $C^{*}$-coactions, Math. Scand. 71 (1992), no. 2, $277-294$. MR 1212711

[25] , Full and reduced $C^{*}$-coactions, Math. Proc. Cambridge Philos. Soc. 116 (1994), no. 3, 435-450, DOI 10.1017/S0305004100072728. MR 1291751

[26] Iain Raeburn and Dana P. Williams, Morita equivalence and continuous-trace $C^{*}$-algebras, Mathematical Surveys and Monographs, vol. 60, American Mathematical Society, Providence, RI, 1998. MR 1634408

[27] Marc A. Rieffel, Applications of strong Morita equivalence to transformation group $C^{*}$-algebras, Operator algebras and applications, Part I (Kingston, Ont., 1980), 1982, pp. 299310. MR 679709

[28] - Proper actions of groups on $C^{*}$-algebras, Mappings of operator algebras (Philadelphia, PA, 1988), Progr. Math., vol. 84, Birkhäuser Boston, Boston, MA, 1990, pp. 141-182. MR 1103376

[29] - Integrable and proper actions on $C^{*}$-algebras, and square-integrable representations of groups, Expo. Math. 22 (2004), no. 1, 1-53. MR 2166968 
Departamento de Matemática, Universidade Federal de Santa Catarina, 88.040-900 FLORIANÓPOLIS-SC, BRAZIL

E-mail address: echters@math.uni-muenster.de

Mathematisches Institut, Westfälische Wilhelms-Universität MÜnster, Einsteinstr. 62, 48149 Münster, Germany 